\title{
Brain-wide connectivity of molecularly defined GABAergic neurons in mouse barrel cortex visualized with optimized rabies virus tracing
}

\author{
Dissertation \\ for the award of the degree \\ "Doctor rerum naturalium" \\ of the Georg-August-Universität Göttingen \\ within the doctoral program IMPRS Neuroscience \\ of the Georg-August University School of Science (GAUSS) \\ submitted by \\ Georg Hafner \\ from \\ Linz, Austria
}

Göttingen, 2019 


\section{Thesis committee}

\section{Referee: Prof. Dr. Jochen F. Staiger}

Institute of Neuroanatomy, University Medical Center, Göttingen

\section{2nd Referee: Prof. Dr. Silvio O. Rizzoli}

Department of Neuro- and Sensory Physiology, University Medical Center, Göttingen

\section{Camin Dean, Ph.D.}

European Neuroscience Institute, Göttingen

\section{Further members of the examination board}

\section{Dr. Marion Silies}

European Neuroscience Institute, Göttingen

\section{Prof. Dr. Nils Brose}

Department of Molecular Neurobiology, Max Planck Institute for Experimental Medicine, Göttingen

Dr. Dr. Oliver Schlüter

Department of Psychiatry and Psychotherapy, University Medical Center, Göttingen 
I hereby declare that this thesis entitled

„Brain-wide connectivity of molecularly defined GABAergic neurons

in mouse barrel cortex visualized with optimized rabies virus tracing" was written by me with no other sources and aids than quoted.

Georg Hafner

$27^{\text {th }}$ March 2019

Göttingen, Germany 


\section{Table of Contents}

1 SUMMARY 1

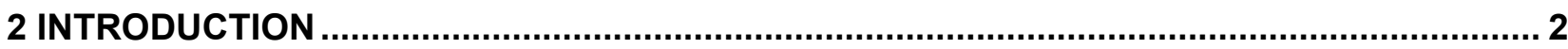

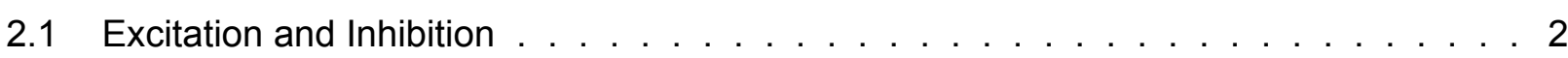

2.1.1 Cell types mediating inhibition in the neocortex. . . . . . . . . . . . 3

2.1.2 PV-expressing inhibitory neurons . . . . . . . . . . . . . . 4

2.1 .3 SST-expressing inhibitory neurons . . . . . . . . . . . . . . . . . . 4

2.1 .4 VIP-expressing inhibitory neurons . . . . . . . . . . . . . . 5

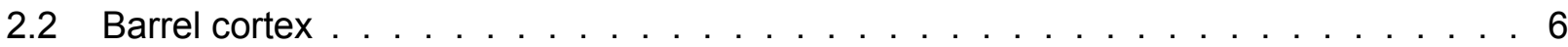

2.3 Viral tracing. . . . . . . . . . . . . . . . . 8

2.4 Tracing brain-wide inputs to cortical GABAergic neurons . . . . . . . . . . . . . 9

2.5 Reeler mouse mutant . . . . . . . . . . . . . . . . . . . . . 9

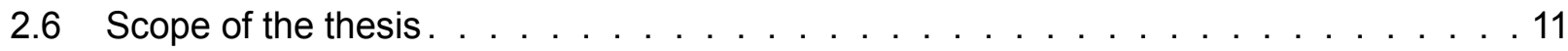

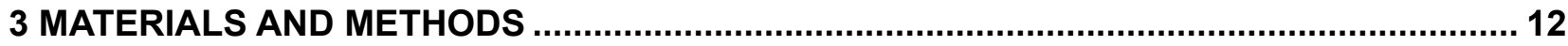

3.1 Animals. . . . . . . . . . . . . . . . . . . . . . . . . . .

3.2 Viral constructs . . . . . . . . . . . . . . . . . . . 12

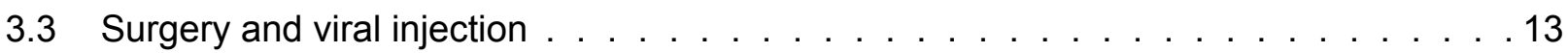

3.4 Intrinsic signal optical imaging (ISOI) $\ldots \ldots \ldots \ldots$

3.5 Fixation and tissue processing . . . . . . . . . . . . . . . . 14

3.6 Immunohistochemistry on tissue sections . . . . . . . . . . . . . . 15

3.7 Fluorescence in-situ hybridization (FISH) on tissue sections . . . . . . . . . . 15

3.8 In vitro electrophysiology and glutamate uncaging. . . . . . . . . . . . 16

3.9 Image acquisition and processing . . . . . . . . . . . . . . . . . . .

3.10 Quantification and statistical analysis . . . . . . . . . . . . . . . 17

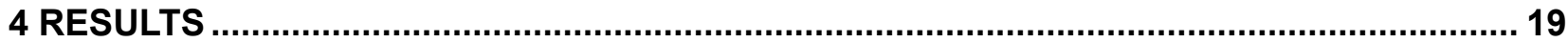

4.1 Intersectional rabies virus tracing of Parvalbumin expressing GABAergic neurons . . . 19

4.1.1 Motivation to use intersectional RV tracing . . . . . . . . . . . . . . . 19

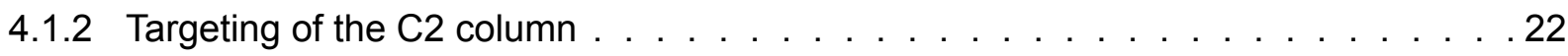

4.1.3 Control experiments reveal a leak of "invisible" TVA . . . . . . . . . . . . . 22

4.1.4 Starter cells are PV-positive and occur in layers II-VI . . . . . . . . . . . . . . 26

4.1.5 The population of PV cells receives predominantly local excitatory inputs . . . . . . 28

4.1.6 LI interneurons inhibit PV neurons in upper LII/III. . . . . . . . . . . . . . . . . . 29 
4.1.7 PV cells receive long-range cortical and subcortical input . . . . . . . . . . . . 32

4.1.8 LIV visual cortex projects to PV neurons in the barrel cortex . . . . . . . . . . . 33

4.2 Comparison of brain-wide inputs to VIP neurons in barrel cortex of WT and reeler mice 35

4.2.1 VIP neurons are misarranged in the reeler mouse $\ldots \ldots \ldots$. . . . . . . . 35

4.2.2 Mutated version of TVA abolishes leak expression of TVA . . . . . . . . . . . 36

4.2.3 Starter cells in reeler do not show a laminar bias . . . . . . . . . . . . . . 40

4.2.4 VIP cells receive qualitatively similar long-range input in WT and reeler . . . . . . .42

4.2 .5 VIP cells in reeler receive less input per cell $\ldots \ldots \ldots$. . . . . . . . . . . . . .

4.2.6 VIP neurons in reeler mice receive more homotopic contralateral input . . . . . . .48

4.2.7 Only contralateral projection neurons in reeler show a different laminar distribution . 48

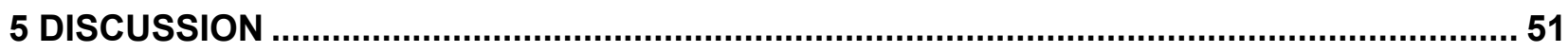

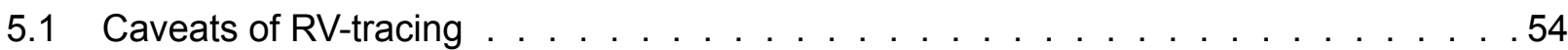

5.2 Control experiments are necessary to assess the cell-type specificity. . . . . . . . .55

5.3 Brain-wide inputs to GABAergic PV cells in mouse barrel cortex . . . . . . . . . . . 56

5.4 Local inputs to PV cells . . . . . . . . . . . . . . . . . . . . . . . . . .

5.5 Long-range input to PV cells . . . . . . . . . . . . . . . . . . . . . . . . . .

5.6 Brain-wide inputs to VIP cells in WT mouse barrel cortex . . . . . . . . . . . . . 58

5.7 Comparison of brain-wide inputs to cortical GABAergic neurons . . . . . . . . . . 59

5.8 Comparison of brain-wide inputs to VIP neurons of WT and reeler mice . . . . . . . 61

5.9 VIP neurons receive less ipsilateral and more contralateral cortical long-range input . . 61

5.10 The proportions of cortical inputs are preserved in reeler with a few exceptions . . . .63

5.11 Distribution of cortical projection neurons in reeler. . . . . . . . . . . . . . 64

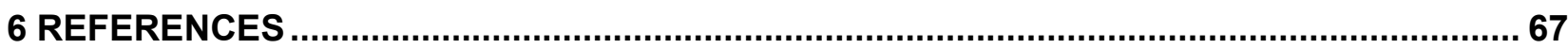

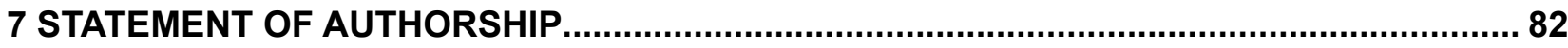

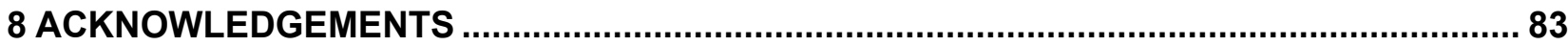

9 PUBLICATIONS ARISING FROM THIS THESIS .......................................................... 84

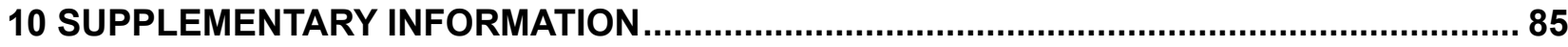

10.1 Tables . . . . . . . . . . . . . . . . . . . . . . . . . . . .

10.2 List of Abbreviations . . . . . . . . . . . . . . . . . . . . . . . . . . . . .

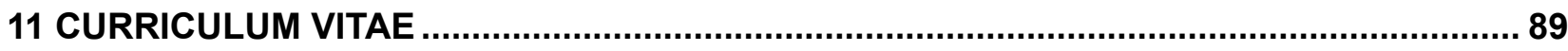




\section{List of Figures}

Figure 1: Major groups of molecularly-defined neocortical GABAergic neurons . . . . . . . . 3

Figure 2: Schematic drawing of the barrel cortex $\ldots \ldots \ldots \ldots$

Figure 3: RV-tracing in PV-Cre line includes excitatory starter cells . . . . . . . . . . . . . 20

Figure 4: Engineering and validation of Cre- AND Flp-dependent AAV-Con/Fon-TVA-mCherry and AAV-Con/Fon-oG . . . . . . . . . . . . . . . . . . . . . . . . 21

Figure 5: Experimental workflow of intersectional RV-tracing of GABAergic PV cells . . . . . 23

Figure 6: Mapping the $\mathrm{C} 2$ whisker representation in barrel cortex . . . . . . . . . . . . . . . 24

Figure 7: Validating intersectional constructs for cell-type specific RV-tracing in vivo . . . . . . 26

Figure 8: Identification of starter cells in Vgat-Cre/PV-Flp mice . . . . . . . . . . . . . . . 27

Figure 9 : Local inputs to PV cells . . . . . . . . . . . . . . . . . . . . 28

Figure 10: LI interneurons provide input to LII/III PV cells . . . . . . . . . . . . . . . . 30

Figure 11: Long-range inputs to PV cells . . . . . . . . . . . . . . . . . 31

Figure 12: Laminar analysis of cortical long-range inputs to PV cells . . . . . . . . . . . . 32

Figure 13: LIV neurons in visual cortex form putative synapses with PV cells in barrel cortex. . 33

Figure 14: Distribution of VIP cells in WT and reeler mice . . . . . . . . . . . . . 35

Figure 15: Experimental workflow of Cre-dependent RV-tracing of VIP neurons . . . . . . . 37

Figure 16: Validation of Cre-dependent constructs for RV-tracing in BL6 animals . . . . . . . . 39

Figure 17: Identification of starter cells in VIP-Cre and VIP-Cre/reeler mice . . . . . . . . . . 41

Figure 18: Long-range input to VIP cells in barrel cortex of WT and reeler mice . . . . . . . . 45

Figure 19: Normalized inputs from global cortical and subcortical areas . . . . . . . . . . . . 46

Figure 20: Comparative analysis of the fraction of inputs from individual areas . . . . . . 448

Figure 21: Distribution of projection neurons in cortical input areas . . . . . . . . . . . . 49

Figure 22: Graphical summary of intersectional RV tracing of GABAergic PV cells in barrel cortex. . . . . . . . . . . . . . . . . . . . . . . . . .

Figure 23: Graphical summary of comparative RV tracing of VIP neurons in the barrel cortex of WT and reeler mice. . . . . . . . . . . . . . . . . . . . 


\section{SUMMARY}

Cortical GABAergic neurons are indispensable in controlling the activity of cortical networks. Parvalbumin (PV), somatostatin (SST) and vasoactive intestinal polypeptide (VIP) expressing neurons are three main classes of GABAergic cells. They differ in morphology, physiology and output connectivity. We wanted to investigate the largely unknown input-connectivity of these neurons on a brain-wide scale using rabies virus tracing. We focused on the PV and VIP neurons in the mouse barrel cortex.

First, we used intersectional rabies virus tracing, to specifically target GABAergic PV cells and exclude a small fraction of excitatory PV cells from our starter cell population. We combined the VgatCre/PV-FIp line with Cre- and Flp-dependent helper viruses. After thoroughly evaluating the specificity of these novel viral constructs, we mapped the local and long-range inputs to PV neurons. Local inputs were mainly from layer ( $L$ ) IV and excitatory. A small number of inputs originated from LI inhibitory neurons, which we found to connect to LII/III PV neurons. Long-range inputs originated mainly from other sensory cortices and the thalamus. Surprisingly, in visual cortex most retrogradely labeled neurons were located in LIV, which sent direct connection to PV cells in all layers of barrel cortex as demonstrated with anterograde tracing experiments.

Second, we assessed the long-range input connectivity of VIP neurons in wildtype and reeler mutant mice, in which layers are not formed during development. VIP neurons received input from the same areas in both genotypes. The major input sources were other sensory cortices, motor cortex, posterior parietal association area and the thalamus. VIP neurons in reeler mice received a much lower number of ipsilateral cortical inputs and a much higher number of contralateral cortical inputs. We hypothesize that the disorganized arrangement of neurons in reeler compromises the establishment of cell-type specific ipsilateral long-range projections and necessitates a compensation by an excess of contralateral inputs.

Both studies provide valuable insights in the brain-wide circuits in which GABAergic neurons are embedded and introduce new and very specific rabies virus tracing tools. 


\section{INTRODUCTION}

Systems neuroscience aims to understand the structure and the function of the nervous system. These two aspects go hand in hand because a system always operates within the constraints of its structure. The nervous system is the one exception amongst all organs for which the relationship between the structure and the function still remains poorly understood. Therefore, tremendous efforts have been launched to map the structure of circuits (Lichtman and Sanes, 2008). The intricate networks of the neocortex are commonly considered the substrate for higher cognitive abilities (Harris and Shepherd, 2015). From their activity arise qualities like unified perception of the environment, evaluation of sensory impressions and motor control. Despite a century of research we are still at dawn of understanding how the cortex generates these qualities.

At the beginning of the analysis of cortical networks is a thorough breakdown of its individual components (Denk and Lichtman, 2011). In cortical networks we can find excitatory and inhibitory cells. Each of these groups exhibit a considerable diversity in structure and function. In pursuit of managing this complexity, neurons are classified into types based on their similarities in morphology, physiology and molecular make-up (Zeng and Sanes, 2017). However, even neurons with similar features can engage in very different actions dependent on how they are wired to other cells. Therefore, the behavior of a neuron is related to its connections with other neurons and thus the connectivity scheme is one of the most important aspects that determines its function. A difficulty in mapping the connectivity scheme is the enormous volume within the tissue that neuronal process span compared to their soma size. Therefore, neuroscientists require techniques that can map connectivity of distinct neuronal types on a large scale. With the advent of cell-type specific tracers from the realm of viral vectors this difficulty can now finally be tackled (Nassi et al., 2015). These tools allow to map brain-wide connectivity of defined types of neurons.

\subsection{Excitation and Inhibition}

A fundamental principle of cortical circuits is the maintenance of a fine-tuned balance of excitation and inhibition (Isaacson and Scanziani, 2011). Inhibition is much more than the guard who keeps epilepsy at bay. It is crucial for every cognitive operation. Just up to $15 \%$ of cortical cells are inhibitory (in primary somatosensory cortex) opposing the great majority of $85 \%$ excitatory cells (Lefort et al., 2009; Meyer et al., 2011). Nevertheless, inhibition dominates excitation in time and amplitude (Haider et al., 2013). To process information, the brain needs to create a controlled imbalance between excitation and inhibition to set a time window in which inputs are integrated to reach the threshold for spike generation (Pouille and Scanziani, 2001). In the end, inhibition determines how many excitatory neurons are recruited so that excitation can spread into the right direction, in the right magnitude and at the right time (Roux and Buzsáki, 2015). 


\subsubsection{Cell types mediating inhibition in the neocortex}

The diversity of inhibitory cell types is key to orchestrate numerous forms of powerful inhibition (Tremblay et al., 2016). Inhibitory cells exhibit a wide range of morphologies, physiological parameters and connectivity schemes (Ascoli et al., 2008; DeFelipe, 2013). However, when taking molecular markers into consideration, inhibitory neuron diversity becomes far more comprehensible. Especially with the advent of single cell transcriptomics it became possible to analyze molecular subtypes of neurons on a large scale (Poulin et al., 2016). The most recent studies point to a classification of GABAergic

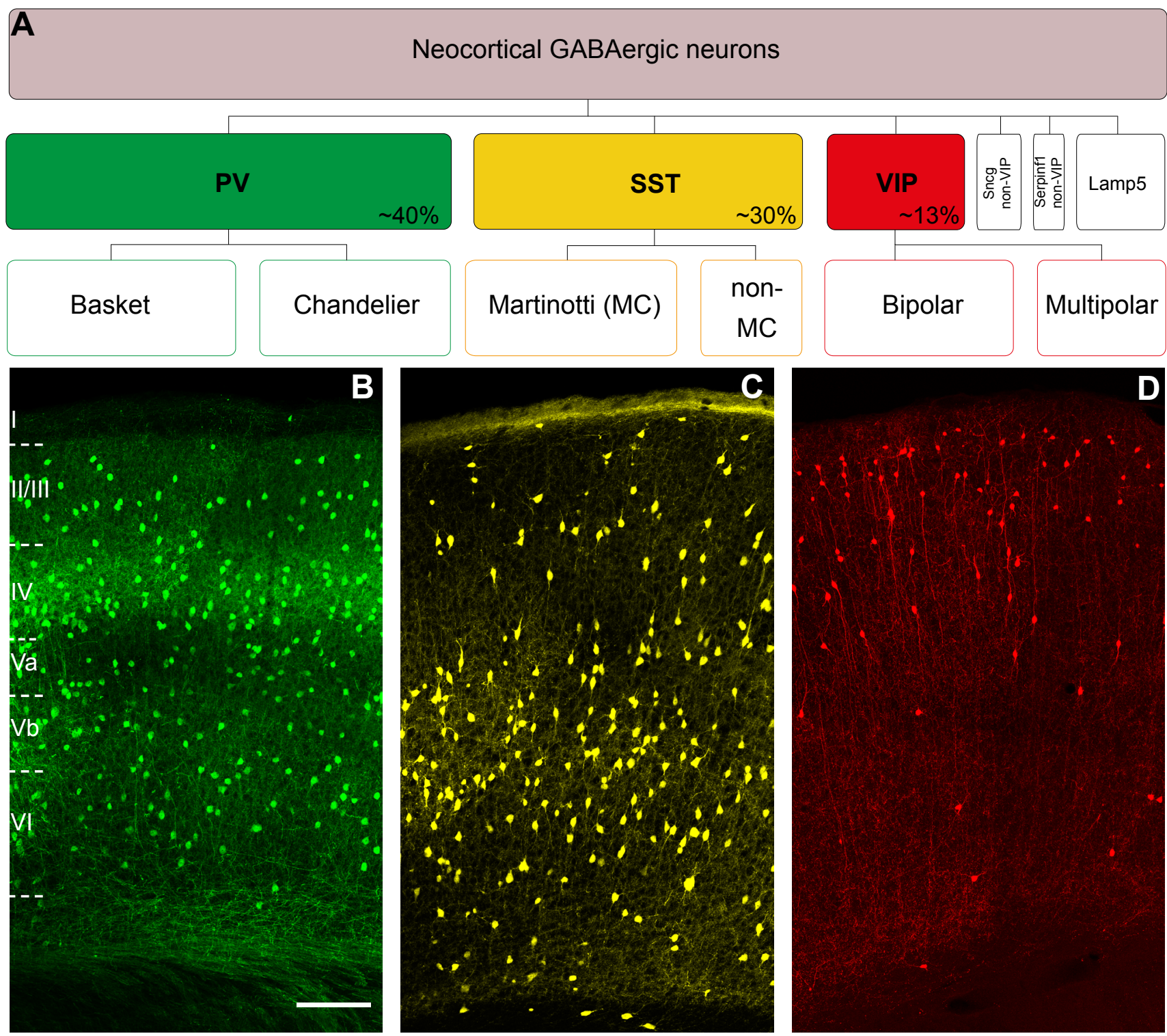

Figure 1: Major groups of molecularly-defined neocortical GABAergic neurons (modified from Staiger et al. (2015))

(A) Parvalbumin (PV), somatostatin (SST) and vasoactive intestinal polypeptide (VIP) expressing neurons make up about $83 \%$ of GABAergic cortical neurons. Each group can be divided into further subgroups based on morphology. PV neurons are distinguished into basket cells and axon-targeting chandelier cells. SST cells are distinguished into Martinotti (MC) and non-Martinotti cells. VIP cells are distinguished into bipolar and multipolar cells. The remaining $17 \%$ of cells express the molecular markers Lamp5, Sncg or Serpinf1.

(B-D) Sections through the barrel cortex in which PV, SST and VIP neurons are labeled. Each of these classes has a typical laminar distribution and morphology (scale bar: $100 \mu \mathrm{m}$ ). 
cells into six major subpopulations (Tasic et al., 2016, 2018): parvalbumin (PV), somatostatin (SST), vasoactive intestinal polypeptide (VIP), Sncg, Serpinf1 and Lamp5 expressing inhibitory cells (Figure $1 \mathrm{~A})$. The first three subtypes make up about $83 \%$ of neocortical GABAergic neurons and are the most studied (Rudy et al., 2011). The generation of Cre-lines for these markers has allowed the specific targeting of defined classes of inhibitory cells and has enhanced the understanding of each cell type fundamentally (Taniguchi et al., 2011).

Noteworthy, previous studies have suggested just three cardinal classes of GABAergic neurons, PV, SST and 5HT3a-R (Lee et al., 2010; Rudy et al., 2011; Vucurovic et al., 2010). However, the latter marker has disappeared in the most recent classification schemes, perhaps because it is only transiently expressed during development and might not have a consistent expression during adulthood.

\subsubsection{PV-expressing inhibitory neurons}

PV-expressing inhibitory neurons are the largest class, comprising $40 \%$ of cortical GABAergic neurons (Celio, 1986; Lee et al., 2010; Pfeffer et al., 2013). They include basket cells and chandelier cells (Tremblay et al., 2016; Figure 1B).

PV cell somata are present in all cortical layers except for LI (Hioki et al., 2013). PV cells usually have an aspiny, multipolar dendritic arbor, which for LIV PV cells is mostly confined to the home layer and column (Koelbl et al., 2015). For upper and deeper layer PV cells the dendrites extend across laminar and columnar borders (Bortone et al., 2014; Packer and Yuste, 2011; Wang et al., 2002). Because of their distribution and their dendritic extent they are ideally suited to sample input from different cortical and subcortical areas and integrate it into the local circuitry (Gonchar and Burkhalter, 1999; Staiger et al., 1996a, 2009). PV basket cells direct their output via an extensive axonal arborization that engulfs postsynaptic cells like a basket, inhibiting mainly the cell soma and proximal dendrites of their postsynaptic targets (Favuzzi et al., 2019; Freund and Katona, 2007). The hallmark of the chandelier cells is their extremely selective innervation of the axon initial segment of pyramidal cells (Somogyi, 1977). PV cells are biologically optimized to provide a very fast, strong and precise inhibition to the postsynaptic cells (Hu et al., 2014). They have been shown to mediate feedforward (Beierlein, 2003; Cruikshank et al., 2010; Pouille and Scanziani, 2001; Pouille et al., 2009) and feedback inhibition (Beierlein, 2003), to modulate the gain of sensory responses (Atallah et al., 2012; El-Boustani and Sur, 2014; Lee et al., 2012; Wilson et al., 2012) and to generate gamma oscillations (Cardin et al., 2009; Sohal et al., 2009). A very important feature for the control of the local network is their global innervation of nearby cells to spread a "blanket of inhibition" over the cortical network and dampen its activity (Karnani et al., 2014, 2016a, 2016b). Finally, PV cells have been associated with plasticity and learning (Donato et al., 2013; Kuhlman et al., 2013; Letzkus et al., 2011; Sparta et al., 2014; Yazaki-Sugiyama et al., 2009).

\subsubsection{SST-expressing inhibitory neurons}

The second largest group of GABAergic cortical cells are with about $30 \%$ the SST expressing neurons (Tremblay et al., 2016; Figure 1C). They are more heterogeneous than PV cells but can be divided 
based on morphology in two main subclasses. Martinotti (MC) and non-MC cells (Figure 1B). The hallmark of MCs is their axon that extends up into $\mathrm{LI}$, which is also the reason why they are commonly believed to inhibit the distal dendrites of pyramidal neurons (Markram et al., 2004; Wang et al., 2004). However, at least LV MC also substantially innervate basal dendrites of pyramidal cells (Hill et al., 2012; Kawaguchi and Kubota, 1997)

Non-MC cells are predominantly constituted by the population of X94-SST cells that have a axon mostly confined to the home layer and are different in electrophysiological properties, too (Ma et al., 2006). MC cells occur in LII-VI, while non-MC cells occur mainly in LIV and V (Tremblay et al., 2016). In both cases, the dendritic processes are much less elaborate and mostly restricted to the home layer (Ma et al., 2006; Muñoz et al., 2017; Nigro et al., 2018; Walker et al., 2016; Xu et al., 2006). Therefore, SST neurons in each layer will receive different inputs and only on the population level they will sample the input to the whole column. SST neurons provide dense inhibition to surrounding excitatory and inhibitory neurons (Chen et al., 2015; Fino and Yuste, 2011; Pfeffer et al., 2013). However, SST neurons never inhibit each other showing a high selectivity for their targets (Hu et al., 2011; Jiang et al., 2015; Karnani et al., 2016a; Ma et al., 2013).

An important property is that they receive facilitating inputs (Urban-Ciecko and Barth, 2016). Thus, SST-mediated inhibition becomes more important in shaping neural responses during conditions of prolonged stimulation (Beierlein, 2003; Kapfer et al., 2007; Pfeffer et al., 2013). SST neuron-mediated inhibition can sharpen orientation tuning of pyramidal neurons (Adesnik et al., 2012) and can modulate the gain of sensory responses without altering tuning properties in visual cortex (Lee et al., 2012). Therefore, SST neurons can engage in different functions dependent on the context (Yavorska and Wehr, 2016). The versatility of SST cells in circuits has also been shown for barrel cortex. While earlier studies have reported a general decrease of SST neuron activity during whisking (Gentet et al., 2012; Lee et al., 2013), a closer look at the subtypes of SST cells distinguished layer-specific differences in the up-or downregulation of SST neurons' activity during whisking (Muñoz et al., 2017). Furthermore, LIV SST neurons seem to engage in different circuit motives than MC. While MC often form disynaptic inhibitory circuits with pyramidal cells for feedback (or lateral) inhibition (Fino and Yuste, 2011; Karnani et al., 2016a; Silberberg and Markram, 2007; Zhang et al., 2014), LIV SST cells inhibit mostly PV cells within this layer pointing to a disinhibitory action (Muñoz et al., 2017; Xu et al., 2013). These results hint at a refined spatiotemporal compartmentalization of SST neuron activity.

Although the role of SST neurons in the cortical network is ambiguous, it seems that in contrast to $\mathrm{PV}$ neurons they contribute more to the fine-tuning of pyramidal neuron activity rather than controlling the magnitude of neuronal responses (Wilson et al., 2012).

\subsubsection{VIP-expressing inhibitory neurons}

VIP neurons make up about 13\% of cortical GABAergic neurons (Prönneke et al., 2015; Tremblay et al., 2016; Figure 1D). VIP cells are found in all cortical layers but they exhibit a clear bias towards LII/ III, where approximately $60 \%$ of all VIP cells are located (Hioki et al., 2013; Prönneke et al., 2015). The morphological diversity of VIP neurons has prevented their classification into clear subtypes (Prönneke et al., 2015), but one morphological group stands out: bipolar/bitufted VIP cells (Bayraktar et al., 2000; 
He et al., 2016; Prönneke et al., 2015). This subtype is most prominent in LII/III. It is characterized by a vertically extending axon that spans the whole column and a vertically oriented dendritic arbor that is mostly confined to $\mathrm{LI}$ and LII/III. The less abundant granular and infragranular VIP cells exhibit a completely different morphology (Prönneke et al., 2015). Their dendritic processes often reach out from the deep home layer up to $\mathrm{LI}$, while their axonal branches stay in the deep layers. The fact that most VIP neurons have a dendrite in LI sets them apart from the other two inhibitory neuron populations that lack a dendrite in LI. Therefore, they have the morphological disposition to receive long-range input that arrives in the fibre-heavy LI (Tremblay et al., 2016). Indeed, VIP neurons have been repeatedly suggested as the major integrators of long-range input both on a morphological (Wall et al., 2016) as well as on a functional basis (Fu et al., 2014; Lee et al., 2013; Zhang et al., 2014).

A very consistent finding is that VIP cell activity is upregulated during active exploration like running or whisking (Dipoppa et al., 2016; Fu et al., 2014; Garcia-Junco-Clemente et al., 2017; Gentet et al., 2012; Lee et al., 2013; Pakan et al., 2016). This upregulation is accompanied by a decrease in SST cell activity (Fu et al., 2014; Gentet et al., 2012; Lee et al., 2013). VIP cells preferentially target SST cells (Caputi et al., 2009; Jiang et al., 2015; Pfeffer et al., 2013; Staiger et al., 2004). SST cells strongly innervate pyramidal neurons (Kapfer et al., 2007; Karnani et al., 2016a; Pfeffer et al., 2013). As a result of VIP activation, SST cells would be deactivated, leading to disinhibition of pyramidal cells. Putting the dots together, VIP neurons become activated by long-range input during active states, disinhibit pyramidal cells and thereby open a precisely timed window for integration and plasticity at excitatory synapses (Pfeffer, 2014; Williams and Holtmaat, 2019). Although this disinhibitory motif is well established and consistently found, some studies hint that it is context dependent and not generic (Dipoppa et al., 2016; Pakan et al., 2016). Furthermore, anatomical evidence suggests that VIP neurons do not exclusively target other inhibitory cells but to a great deal excitatory cells, too (Zhou et al., 2017). Garcia-Junco-Clemente et al. (2017) showed that in frontal cortex VIP neurons engage both in disinhibition and direct inhibition of pyramidal cells. Therefore, VIP neurons might have dual action subjecting pyramidal neurons to a push-pull inhibitory-excitatory circuit during arousal (Garcia-Junco-Clemente et al., 2017).

In sum, these three inhibitory neuronal subtypes participate in various cortical operations with overlapping but also distinct forms of inhibition. Which type of neuron is more strongly recruited is highly context dependent. If we want to understand in which context certain classes of inhibitory cells are activated, we first need a comprehensive, brain-wide and cell-type specific map of their potential inputs.

\subsection{Barrel cortex}

These inhibitory neurons, just like the excitatory cells, appear in the whole neocortex. They are the building blocks of circuits in each neocortical brain area. In what numbers they occur and how they are connected generates the functional specialization of a cortical area. Therefore, the circuitry a certain neuronal types is embedded in has to be studied in the scope of its functional area. We focused on the vibrissal area of the primary somatosensory cortex of mice for several reasons.

Active touch is a common behavior to interact with the environment. Mice use whiskers on their 

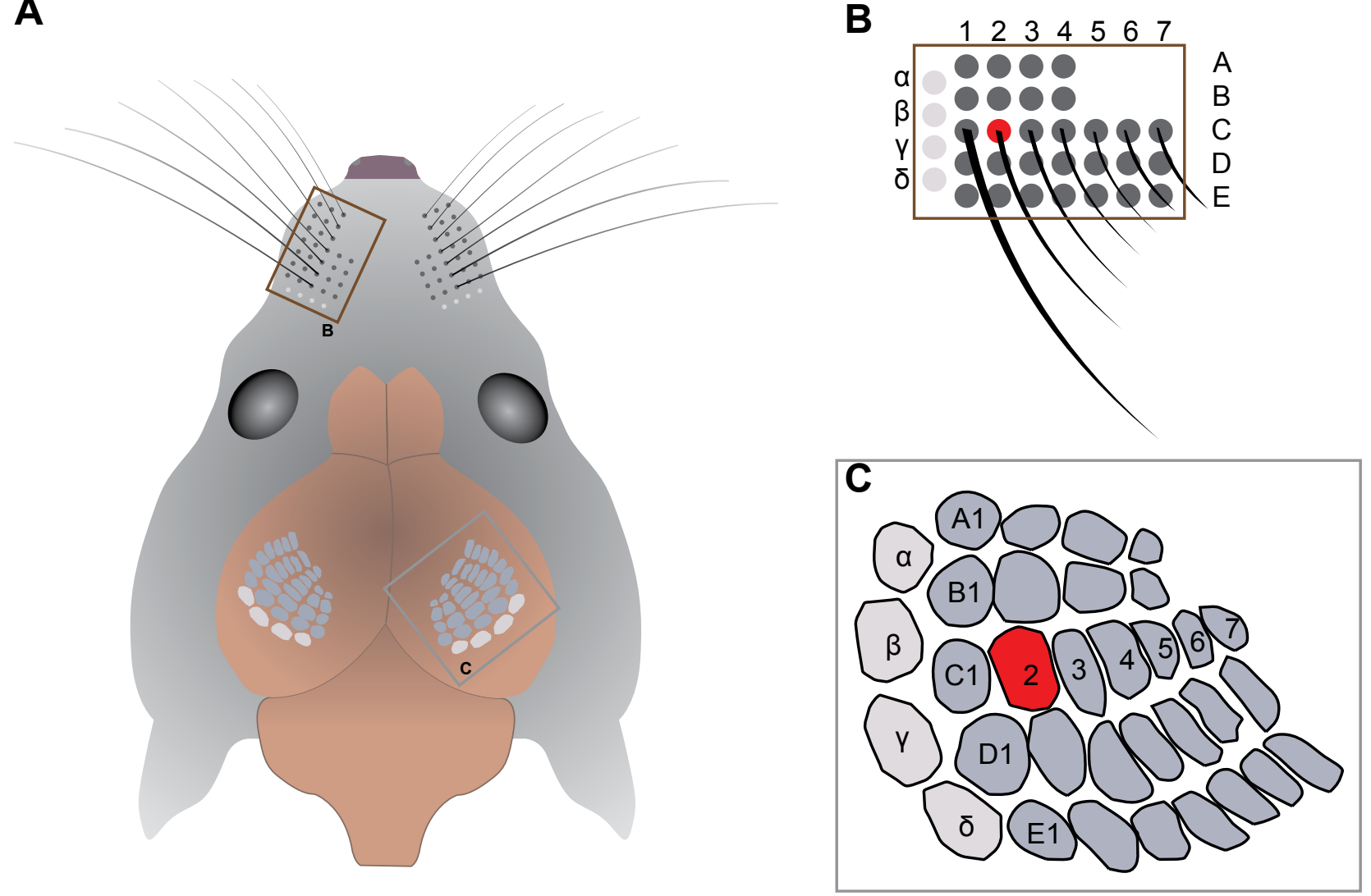

Figure 2: Schematic drawing of the barrel cortex

(A) Drawing of mouse head showing the position of whisker follicles on the muzzle and of the barrel cortex in the brain.

(B) The arrangement of whisker follicles mirrors the arrangement of barrel-related columns in the cortex. Arcs of whiskers are denoted by numbers while rows of whiskers are denoted by letters. The straddlers are denoted by Greek symbols. Similarly, this code is used for the barrel related cortical columns, so that each whisker can be related to its corresponding cortical representation.

muzzle to explore their environment, locate objects and discern their shape, size and texture. Whisker based touch is a very important sensory modality for mice, already indicated by the large size of the respective cortical area dedicated to processing this kind of information (Figure 2A). The so-called "barrel cortex" contains distinct barrel-shaped structures in LIV (Woolsey and Loos, 1970). These barrels are somatotopically arranged mirroring the arrangement of the whiskers (Figure 2B, C). Each barrel represents the input structure for sensory information from one corresponding whisker on the contralateral side. This somatotopy is preserved at every relay station of the whisker-to-barrel pathway. Sensation starts at the whisker follicles. They are loaded with mechanoreceptive nerve endings (Ebara et al., 2002). These sensory neurons synapse onto neurons in the trigeminal pontine nuclear complex, where the neuronal modules representing individual whiskers are arranged into "barrelettes" (Ma and Woolsey, 1984). From there information is relayed via the lemniscal pathway to the map of "barreloids" in the ventral posteromedial (VPM) nucleus of the thalamus (Chmielowska et al., 1989). The thalamocortical terminations form the dense core of the cortical "barrel" which is enclosed by a cell body rich barrel wall (Woolsey and Loos, 1970). The barrel is an anatomical landmark for the barrel-related column that stretches from the pia to the white matter. Neurons within this barrel-related column respond most strongly to stimulation of the corresponding whisker (Simons and Woolsey, 1979). To ease the annotation of whiskers and barrels they have been assigned a code of letters and numbers. 
Each whisker row is labeled from A-E, each whisker arc is numbered. The cortical barrels are labeled the same way (Figure 2B, C).

In the barrel cortex the functional module for processing of sensory information is visible as an anatomical module. Therefore, this model systems is ideal to investigate the relationship between structure and function. The barrel cortex has become a well-studied area so that a large amount of data on cellular composition and interareal connectivity is already available (Brecht, 2007; Feldmeyer et al., 2013, 2017, 2018; Petersen, 2007). For these reasons, it is an excellent area to study the brain-wide circuitry of defined neuronal types.

\subsection{Viral tracing}

Conventional anatomical tracers like biotinylated dextran amines, wheat germ agglutinin, fluoro-gold or cholera toxin B fragment have been used extensively to map the projections of and the inputs to neurons. They have greatly enhanced our understanding about the interconnectivity of brain areas. These tracers have the fundamental limitation that they do not reveal which kinds of cell types these traced axons innervate. Connections among neurons - no matter if they are local or across wide distances - are not random but highly selective for their targets (Brown and Hestrin, 2009; Helmstaedter et al., 2013; Perin et al., 2011; Silberberg et al., 2002; Song et al., 2005). Cellular identity rather than close proximity of processes is relevant for generation of connections (Kasthuri et al., 2015). In consequence, tracers need to have sufficient resolution to probe cell-type specific connections, hence they need to be transneuronal (Callaway, 2008). Neurotropic viruses can be used as transneuronal tracers. They are perfectly adapted to infect neurons, multiply and spread (Kuypers and Ugolini, 1990). Among transneuronal tracers rabies virus (RV) has built a reputation as the most specific one (Ugolini, 2010).

$\mathrm{RV}$ is a $180 \mathrm{~nm}$ small, bullet shaped virus (Dietzschold et al., 2008). It has several features that make it the ideal retrograde, transsynaptic tracer. (I) RV spreads selectively between synaptically connected neurons and does not label axons of passage (Callaway and Luo, 2015; Ugolini, 1995). (II) RV labels presynaptic cells regardless of the distance of their soma location making it an ideal tracer for long-range projections (Callaway, 2008; Kelly and Strick, 2000; Wickersham et al., 2007a). (III) RV amplifies very strongly within the cell and strongly labels its whole volume, even if the cell is a weakly connected input (Loewy, 1998; Wickersham and Feinberg, 2012). (IV) RV has - compared to other neurotropic viruses - a surprisingly low cytotoxicity. For up to 12 days the viability of the neuron stays unharmed (Osakada et al., 2011; Wickersham et al., 2007b). This is ample time for RV to spread and brightly label presynaptic partners.

These features motivated scientists to tame RV and use it as a transneuronal tracer. Deletion of its glycoprotein $(\mathrm{G})$ allowed RV to be safely used in a laboratory environment and to restrict its spread to first-order presynaptic neurons (Mebatsion et al., 1996). The combination of RV with Cre-dependent AAV helper viruses allowed to exploit the vast number of available Cre-mouse lines for monosynaptic circuit tracing of distinct cell types (Callaway and Luo, 2015; Wickersham et al., 2007a). In consequence, RV tracing constitutes the ideal approach to visualize brain-wide inputs to a defined class of neurons. 


\subsection{Tracing brain-wide inputs to cortical GABAergic neurons}

Do PV, SST and VIP cells in barrel cortex receive different sources of brain-wide inputs? This was a central question at the beginning of this $\mathrm{PhD}$. It was motivated by the fact that each of these neuron types shows a different morphology, laminar distribution, intrinsic properties and putative function in a given behavioral context. Therefore, it was speculated that these neurons might receive input from different areas or in different proportions. With the development of the RV-tracing approach it became feasible to study this question (Wall et al., 2010; Wickersham et al., 2007a). One study in particular has looked at the brain-wide inputs to PV, SST and VIP cells in barrel cortex during the time of this thesis work (Wall et al., 2016). The authors found no qualitative difference in the sources of inputs. However, they found that VIP neurons receive more long-range input per cell and more long-range input from the infragranular cortical layers than PV or SST cells. These results demonstrated that the quantitative differences in input can be very subtle. Therefore, we emphasize in this work the importance of very specific and effective tools to trace inputs so that the very minute differences in anatomical long-range connectivity can be revealed. In cooperation with others we generated very specific tools for Cre-dependent and for Cre- and Flp-dependent RV-tracing. These tools allowed us to tackle two very different projects concerned with the topic of inputs to GABAergic cells.

The first project was motivated by our finding that the neurons labeled in the conventionally used PV-Cre line are not exclusively inhibitory. This has been noticed by others (Madisen et al., 2015; Wall et al., 2010) but was never thoroughly examined nor made a point of concern for experiments. In our pursuit of a truly cell-type specific tracing we used an intersectional RV-tracing approach to isolate the GABAergic population of PV cells and label their inputs.

The second project revolved around the question if the structural organization of the cortex into layers is a necessity to properly wire neurons into their circuits. We focused on VIP neurons because of their distinct laminar arrangement and labeled their inputs with an improved Cre-dependent RV tracing approach in wildtype (WT) and reeler mutant mice.

\subsection{Reeler mouse mutant}

A fundamental feature of cortical organization is the arrangement of neurons into distinctive layers. Most of the neocortex can be divided into six layers, each of them defined by a unique composition and density of cell types. Furthermore, each layer has a distinct input-output connectivity so that the flow of information runs across the layer compartments in a stereotypical fashion (Douglas and Martin, 2004). It is unclear what exactly the significance of this layer arrangement is for cortical processing. Cortical computations require foremost a functional neuronal circuit between defined types of cells (Guy and Staiger, 2017; Harris and Shepherd, 2015). The connectivity rules might stay conserved no matter if the cell classes are arranged into layers or intermingled (Harris and Shepherd, 2015).

The reeler mouse mutant has become a model that questions the necessity of layers (Guy and Staiger, 2017). The reeler mutation appeared spontaneously in a stock of inbred mice and induces a loss of reelin protein expression. Homozygous mice have a characteristic reeling gate resulting from an 
uncoordinated movement of their hindquarters (Falconer, 1945). While this phenotype is due to a severe atrophy of the cerebellum, the reeler mutation also affects the lamination of the cortex (Hamburgh, 1960, 1963). During cortical development, reelin is secreted by Cajal-Retzius cells in the marginal zone (Bock and May, 2016; Lee and D'Arcangelo, 2016). It guides the proper migration of neurons to form layers in an inside-out pattern (Kubo and Nakajima, 2003). In the absence of reelin the migration is perturbed and layers do not form. Instead, cells end up in ectopic positions (Boyle et al., 2011; Dekimoto et al., 2010; Pielecka-Fortuna et al., 2014; Wagener et al., 2010). The pattern of disorganization even varies among cortical areas. Motor cortex and rostral S1 show a mixture of chaotic dispersion of cells and normal migration (Boyle et al., 2011; Dekimoto et al., 2010). Caudal S1 shows a complete disorganization of layer fate neurons (Boyle et al., 2011; Wagener et al., 2010). Visual cortex shows a tendency towards inversion of layers (Boyle et al., 2011; Pielecka-Fortuna et al., 2014).

Despite this disorganized arrangement of cells, the reeler mouse shows no severe decline in cognitive abilities. They have normal sensory function in the olfactory, auditory and visual modality (Pielecka-Fortuna et al., 2014; Salinger et al., 2003). They have equal learning ability when using visual information to form behavioral decisions (Pielecka-Fortuna et al., 2014). They have reduced spatial memory and abnormal executive function (Salinger et al., 2003). Despite some deficits, the reeler mouse shows much better abilities than one would expect from a mouse with a completely disorganized cortex.

This has raised the question if neuronal circuits are composed similarly in the absence of layers and if so how this is achieved. Several studies have investigated the connectivity scheme in the reeler mouse. Especially for the cortico-thalamic fibers it has been consistently shown that they find their ectopic cortical targets (Caviness and Frost, 1983; Guy et al., 2016; Harsan et al., 2013; Wagener et al., 2015). Similarly, cortical neurons maintain proper projections as has been shown for the projection from entorhinal cortex to the hippocampal formation (Stanfield and Cowan, 1979), the projection from piriform cortex to the medial prefrontal cortex and the entorhinal cortex (Diodato et al., 2016), as well as callosal connections between homotopic areas. During tactile exploration the same cell types in reeler and WT are activated suggesting the activation of the same functional network (Wagener et al., 2015). Even in a dislaminated cortex, neurons become incorporated in similar circuits and integrate the same sources of local and long-range inputs (Caviness, 1976).

However, none of these studies have assessed if the same number of connections or the same proportions of different input sources on individual cell types are maintained. Therefore, we wanted to investigate if the cell-type specific long-range inputs to VIP neurons in the barrel cortex of reeler mice are qualitatively and quantitatively similar to WT. We decided to probe VIP neurons because they have this very typical laminar bias towards the supragranular layers as described above. Therefore, we first confirmed that VIP neurons in reeler mice lack this laminar bias and instead are distributed fairly homogenously throughout the cortical thickness. In consequence, we could use RV tracing to study the question if the VIP neurons are still embedded in the same circuits despite their malposition. 


\subsection{Scope of the thesis}

The purpose of this thesis was to gain knowledge about brain-wide inputs to GABAergic neurons in the barrel cortex. With the advent of viral tracing approaches it became possible to asses connectivity on a cell-type specific and brain-wide level. We made use of this approach to study two questions:

1) What are the brain-wide monosynaptic inputs to GABAergic PV neurons in the mouse barrel cortex?

2) Is the long-range connectivity of VIP neurons different in reeler mouse mutants who are devoid of layers?

The main approach we took for each project was RV-tracing. In both cases we designed new viral tools in cooperation with others that allowed us to trace cell-types with unprecedented specificity and were optimally tuned to study our questions.

Furthermore, we will discuss the question if the three main types of GABAergic neurons - PV, SST, VIP-expressing - receive different long-range inputs. This was a central question at the beginning of this thesis, which has been addressed by another study (Wall et al., 2016). We will put our findings in the context of this and several other cell-type specific tracing studies of inhibitory cortical neurons. 


\section{MATERIALS AND METHODS}

\subsection{Animals}

We crossed Vgat-ires-Cre mice (Slc32a1tm2(cre)Lowl/J, The Jackson Laboratory, Bar Harbor, USA) with Pvalb-T2A-FlpO-D mice (B6.Cg-Pvalbtm4.1(flpo)Hze/J, The Jackson Laboratory) to generate Vgat-Cre/PV-FIp mice for intersectional RV tracing. We crossed Vgat-Cre/PV-Flp mice with the Creand Flp-dependent Ai65 tdTomato reporter line (B6;129S-Gt(ROSA)26Sortm65.1(CAG-tdTomato) $\mathrm{Hze} / \mathrm{J}$, The Jackson Laboratory), to visualize the intersectional population.

For anterograde tracing of LIV cells, we used Scnn1a-Tg3-Cre (B6;C3-Tg(Scnn1a-cre)3Aibs/J, The Jackson Laboratory).

For Cre-dependent tracing experiments we used B6 PV-Cre mice (B6;129P2-Pvalbtm1(cre)Arbr/J, The Jackson Laboratory). For glutamate uncaging, 3-6 weeks-old offspring of PV-Cre mice crossed to the Ai9 tdTomato reporter mice (B6.Cg-Gt(ROSA)26Sortm9(CAG-tdTomato)Hze/J, The Jackson Laboratory) were used.

For Cre-dependent tracing in reeler mice, we crossed the B6C3Fe line (The Jackson Laboratory) with the VIP-Cre (VIPtm1(cre)Zjh, The Jackson Laboratory) to breed VIP-Cre/reeler animals heterozygous for reelin mutation and homozygous for Cre. These animals were crossed to generate VIP-Cre/ reeler animals homozygous for reelin. WT littermates or animals from the VIP-Cre line were used for comparison in tracing experiments.

For control experiments, we used either single transgenic mice Vgat-Cre and PV-FIp or C57BL/6J wildtype mice (The Jackson Laboratory).

All mice were kept in standard cages in a 12h light/dark cycle and with ad libitum access to food and water. All experimental procedures were performed in accordance with German laws on animal research (TierSchG und TierSchVersV 2013). All tracing experiments were performed with 12-20 weeksold mice of either gender.

\subsection{Viral constructs}

AAV8-nEF-Con/Fon-TVA-mCherry and AAV8-EF1a-Con/Fon-oG were used together with RV-SAD $\Delta$ GEGFP (EnvA). Cre-and-Flp-dependent versions of oG and TVA-mCherry were developed using previously described methods (Fenno et al., 2014, 2017) by Lief Fenno, Charu Ramakrishnan, Yoon S. Kim and, Karl Deisseroth (Stanford University). For cortical injections 200-300 nl of AAVs were injected with a $1: 1$ ratio using a titer of $5^{\star} 10^{\wedge} 12 \mathrm{IU} / \mathrm{ml}$.

We tested several Cre-dependent AAV helper viruses who were kindly donated by our collaborators mentioned below. They were used together either with RV-SAD $\triangle$ G-EGFP (EnvA) if they express mCherry or together with RV-SAD $\Delta$ G-mCherry (EnvA) if they express EGFP. For both RVs we used titers of $1^{*} 10^{\wedge} 7 \mathrm{IU} / \mathrm{ml}$. RV was kindly donated by Karl-Klaus Conzelmann (LMU Munich). 
AAV1-FLEX-TVA-EGFP-G, AAV1-FLEX-tTA-EGFP-TVA and AAV1-TREtight-BFP-G were donated by lan Wickersham (Massachusetts Institute of Technology). For cortical injections 200-300 nl of AAVs were injected. AAV1-FLEX-TVA-EGFP-G was used at a titer of $4{ }^{*} 10^{\wedge} 12 \mathrm{lU} / \mathrm{ml}$. AAV1-FLEX-tTAEGFP-TVA and AAV1-TREtight-BFP-G were injected at a $1: 1$ ratio with a titer of $2^{*} 10^{\wedge} 12 \mathrm{IU} / \mathrm{ml}$.

AAV1-FLEX-TVA-mCherry and AAV8-FLEX-oG were donated by Karl Deisseroth (Stanford University). For cortical injections $200-300 \mathrm{nl}$ of AAVs were injected at a $1: 1$ ratio with a titer of $5^{\star} 10^{\wedge} 12$ $\mathrm{IU} / \mathrm{ml}$.

AAV1/2-FLEX-mCherry and AAV1/2-FLEX-G-ires-TVA were donated by Martin Schwarz (University of Bonn). For cortical injections $200-300 \mathrm{nl}$ of AAVs were injected at a 1:1 ratio with a titer of $5^{\star} 10^{\wedge} 11 \mathrm{IU} / \mathrm{ml}$.

AAV8-FLEX-TVA ${ }^{66 T}$-EGFP-oG, AAV1-FLEX-TVA ${ }^{66 T}$ and AAV8-FLEX-oG were donated by Edward Callaway (SALK Institute). For cortical injections 30-300 nl of AAVs were injected. AAV8-FLEX-TVA ${ }^{66 T}$ EGFP-oG was used at a titer of $1.6^{*} 10^{\wedge} 13 \mathrm{IU} / \mathrm{ml}$.

AAV2/1-CAG-FLEX-tdTomato for visual cortex injections was obtained from PENN Vector core. $400 \mathrm{nl}$ with a titer of $1^{*} 10^{\wedge} 13 \mathrm{IU} / \mathrm{ml}$ were injected.

\subsection{Surgery and viral injection}

All animals subjected to RV tracing underwent surgery for intrinsic signal optical imaging (ISOI), with subsequent mapping of the $\mathrm{C} 2$ whisker-related column.

For sedation and analgesia, the mouse was injected intraperitoneally with $10 \mu \mathrm{g} / \mathrm{g}$ xylazine (Xylariem, Ecuphar) and $0.065 \mu \mathrm{g} / \mathrm{g}$ buprenorphine (Temgesic, Individor UK Limited) in sterile saline. Anesthesia was induced with $3 \%$ isoflurane ( $\mathrm{vol} / \mathrm{vol}$ ) and maintained between 0.5 and $1 \%$ throughout the surgery (Harvard Apparatus, USA). The mouse was mounted on a custom-built frame with rigid earbars. A mixture of $2 \mu \mathrm{g} / \mathrm{g}$ bupivacaine/lidocaine (Astra Zeneca) was injected subcutaneously under the scalp for local anesthesia. Body temperature was maintained at $37^{\circ} \mathrm{C}$ with a heating pad (ATC 1000, World Precision Instruments, Florida). A small incision was made in the scalp to expose the right hemisphere of the skull. The bone over the somatosensory area was thinned to transparency with a dental drill (OS-40, Osada Electric Company, Japan). Then the location of the C2-related column was determined and mapped on the blood vessel pattern as described below. The bone above the target area was removed with a syringe tip. A glass injection pipette cut to $20 \mu \mathrm{m}$ tip diameter (Drummond Scientific Co, USA) was backfilled with mineral oil (Sigma-Aldrich) and front filled with AAV helper virus(es). The pipette was fitted into a Nanoject III (Drummond Scientific Co, USA), which was attached to a micromanipulator (Luigs \& Neumann, Germany). The pipette was inserted the target location into the brain in an approximately $45^{\circ}$ angle, orthogonal to the curvature of the cortex. 200-300 $\mathrm{nl}$ of AAV mixture was injected portioned across three depths $(750 \mu \mathrm{m}, 500 \mu \mathrm{m}$, and $250 \mu \mathrm{m}$ below pia). The needle was left in place at each depth for at least three minutes to reduce backflow. The scalp was sutured and the mouse received a subcutaneous injection of $5 \mu \mathrm{g} / \mathrm{g}$ Carprofen (Pfizer) for prolonged pain relief. 15-20 days later, the mouse was injected with RV without prior imaging. The injection was guided based on the blood vessel pattern and landmarks from the previous surgery. 
For control injections in barrel cortex and for visual cortex injections, mice underwent stereotaxic surgery based on Atlas coordinates (Paxinos and Franklin, 2001). The surgery was performed as for ISOI, with the following changes. The mouse was mounted on a stereotaxic frame (Kopf, Germany). The injection pipette was targeted at $3.3 \mathrm{~mm}$ lateral and $-1.55 \mathrm{~mm}$ caudal from Bregma for barrel cortex and at 2.0 and $2.8 \mathrm{~mm}$ lateral and 3.5 caudal from Bregma for visual cortex.

\subsection{Intrinsic signal optical imaging (ISOI)}

The whiskers on the left side of the snout were clipped, sparing solely C2. This whisker was inserted into a glass capillary glued to a piezo actuator, which was connected to a computer-controlled amplifier (E-650 LVPZT, Physik Instrumente, Germany). The optical system consisted of a CCD camera (Adimec-1000m, Adimec, Netherlands) attached to an objective (Nikon, Japan) composed of two lenses, a $50 \mathrm{~mm}$ bottom lens and a $135 \mathrm{~mm}$ top lens. This system yielded a magnification of 2.7 fold. The camera had a resolution of $1000 \times 1000$ pixels each sized $2.8 \times 2.8 \mu \mathrm{m}$. The Imager 3001 data acquisition system (Optical Imaging, Israel) was connected to the optical system, the piezo amplifier and a computer, controlling the timing of stimulation and data acquisition. Localization of the $\mathrm{C} 2$ column was performed similar to Guy et al. (2014). The thinned skull was moistened with a $0.9 \%$ saline and covered with a glass coverslip (Thermo Scientific Menzel-Gläser, Germany). The camera was focused on the pial surface and the skull was evenly illuminated with green light at $546 \mathrm{~nm}$ wavelength from a $100 \mathrm{~W}$ halogen lamp (Kepco ATE 15-15 M, Kepco, Japan) to visualize the blood vessel pattern. For functional imaging, the plane of focus was moved $300 \mu \mathrm{m}$ below the pial surface, the illumination was switched to red light at $630 \mathrm{~nm}$ wavelength by changing the filter, and the light intensity was adjusted to almost saturate the camera. Stimulation of the $\mathrm{C} 2$ whisker was achieved by bending a piezo element 5 times per second for $10 \mathrm{~ms}$ with an amplitude of approximately $1 \mathrm{~mm}$. $40 \mathrm{~ms}$ long frames were acquired after stimulus onset for $3 \mathrm{~s}$. 30 trials were averaged to improve signal to noise ratio. The blood vessel pattern and the intrinsic signal were overlaid to guide the subsequent injection. The peak of the signal was assumed to correspond to the center of the cortical C2 representation.

\subsection{Fixation and tissue processing}

Eight to ten days after RV injection, mice were sacrificed with an overdose of ketamine (100 $\mu \mathrm{g} / \mathrm{g}$; Medistar) and perfused transcardially with $10 \%$ sucrose solution followed by $4 \%$ paraformaldehyde (PFA) in $0.1 \mathrm{M}$ phosphate buffer saline (PB). The brain was postfixed in $4 \%$ PFA for $4 \mathrm{~h}$ and drowned in $20 \%$ sucrose in $0.1 \mathrm{M}$ PBS overnight. Fixed brains were quick-frozen in $-40^{\circ} \mathrm{C}$-cold isopentane and stored at $-80^{\circ} \mathrm{C}$, until they were embedded in Tissue-Tek O.C.T. compound (Sakura Finetek) and sectioned on a cryostat (CM3050S, Leica). Whole brains were cut into $100 \mu \mathrm{m}$-thick coronal sections rostral and caudal to barrel cortex, while barrel cortex was cut into 40 or $50 \mu$ m-thick sections. All sections spanning the barrel cortex were subjected to immunohistochemistry or fluorescence in-situ hybridization, while all other sections were stained for 4‘,6-diamidino-2-phenylindole (DAPI) only. 


\subsection{Immunohistochemistry on tissue sections}

Barrel cortex sections were washed in TRIS buffer (TB) for $15 \mathrm{~min}$, TRIS-buffered saline (TBS) for 15 min and TBS $+0.5 \%$ Triton X-100 (TBST) for $2 \times 15$ min, all at $\mathrm{pH} 7.6$. Blocking was done for $90 \mathrm{~min}$ at room temperature in $0.25 \%$ bovine serum albumin/10\% goat serum/TBST (Jackson Immuno Research). Sections were incubated for $48-72 \mathrm{~h}$ at $4^{\circ} \mathrm{C}$ with primary antibodies (i) chicken anti-GFP (Aves) diluted 1:500, (ii) mouse anti-RFP (Rockland) diluted 1:1000 (or rabbit anti-RFP (Rockland), and (iii) rabbit anti-PV (Swant) diluted 1:5000 in blocking solution. After washing 4×15 min with TBST, secondary antibodies (i) Alexa Fluor 488-conjugated goat anti-chicken IgG, (ii) Alexa Fluor 568-conjugated goat anti-mouse IgG2a (or Alexa Fluor 594-conjugated goat anti-rabbit), and (iii) Alexa Fluor 633-conjugated goat anti-rabbit (Molecular Probes) were diluted 1:500 in TBST and sections were incubated for $4 \mathrm{~h}$ at room temperature. After washing $2 \times 15$ min with TBST and $1 \times 15$ min with TBS, sections were stained with DAPI, diluted 1:1000 in TBS. After several washes in TB, sections were mounted in Aqua-Poly-Mount (Polysciences).

\subsection{Fluorescence in-situ hybridization (FISH) on tissue sections}

rRNA probes were generated as described in (Prönneke et al., 2015), based on published primers: Slc17a7(vGluT1, vesicular glutamate transporter 1), SST, VIP (Prönneke et al., 2015), and GAD67 (glutamic acid decarboxylase 1; Weissbourd et al., 2014).

Sections spanning the injection site were selected and treated with $1 \% \mathrm{H} 2 \mathrm{O} 2$ in methanol for 20 min, rinsed in PBS, quenched in $0.2 \mathrm{M} \mathrm{HCl}$ for $8 \mathrm{~min}$, rinsed with PBS, incubated in Proteinase $\mathrm{K}$ (10 $\mu \mathrm{g} / \mathrm{mL}$, Roche) in TRIS-HCl/EDTA (50 mM/5 mM, pH 8.0) for $8 \mathrm{~min}$, rinsed with PBS and fixed again in $4 \%$ PFA for $20 \mathrm{~min}$. Sections were directly transferred to $0.25 \%$ acetic anhydride $(2.4 / \mu \mathrm{L}$ per $\mathrm{mL}$ $0.1 \mathrm{M}$ triethanolamine $/ \mathrm{HCl})$ for $10 \mathrm{~min}$, rinsed twice in $2 \times$ standard saline citrate $(1 \times \mathrm{SSC}: 0.15 \mathrm{M} \mathrm{NaCl}$, $0.015 \mathrm{M}$ sodium citrate, $\mathrm{pH} 7.0$ ) and pretreated in hybridization buffer (HB; 50\%formamide, $4 \times$ SSC, $250 \mu \mathrm{g} / \mathrm{mL}$ of denaturated salmon sperm DNA, $100 \mu \mathrm{g} / \mathrm{mL}$ of tRNA, $5 \%$ dextransulfate, and $1 \%$ Denhardt's solution) diluted $1: 2$ with $2 \times$ SSC for 15 min followed by $1 \mathrm{~h}$ of prehybridization at $55^{\circ} \mathrm{C}$ in pure HB. DIG- labeled RNA probes $\left(200 \mathrm{ng} / \mathrm{mL}\right.$ ) were heated to $95^{\circ}$ for $5 \mathrm{~min}$ and added to each well. After hybridization over night at $55^{\circ} \mathrm{C}$, section were washed in $2 \times \mathrm{SSC}$ for $15 \mathrm{~min}$ at RT, 2× SSC containing $50 \%$ formamide for $30 \mathrm{~min}$ at $65^{\circ} \mathrm{C}$, and $2 \times \mathrm{SSC}$ for $5 \mathrm{~min}$ at $65^{\circ} \mathrm{C}$, always twice, followed by RNase $\mathrm{A}$ treatment (Roche, $4 \mu \mathrm{g} / \mathrm{mL}$ ) and washes in $2 \times$ SSC for $5 \mathrm{~min}$ at RT, $2 \times$ SSC containing $50 \%$ formamide for $30 \mathrm{~min}$ at $65^{\circ} \mathrm{C}, 0.1 \times$ SSC containing $50 \%$ formamide for $15 \mathrm{~min}$ at $65^{\circ} \mathrm{C}$, and finally $0.1 \times \mathrm{SSC}$ for $2 \times 15 \mathrm{~min}$ at $65^{\circ} \mathrm{C}$. The hybridized probe was detected using a using a Tyramide Signal Amplification Kit (TSA Biotin System NEL700001KT; PerkinElmer). First, sections were blocked in $0.5 \%$ blocking agent and $4 \%$ sheep serum in $0.01 \mathrm{M}$ TBS, $\mathrm{pH} 7.5$, followed by incubation with sheep anti-DIG-POD FAB fragments, conjugated to peroxidase (Roche) diluted 1:2000 in blocking agent overnight. After biotinylation in biotinyl tyramide working solution for $2 \mathrm{~h}$, rinsing in TBS, Streptavidin Alexa Fluor 633 (Life technologies; diluted 1:400 in TBS, 15min) was used, to fluorescently label the mRNA of interest.

Subsequent immunoamplification of the GFP and mCherry signals was done with goat anti-GFP (Abcam) diluted 1:1000 and rabbit anti-RFP (Rockland) diluted 1:250, following the protocol for immu- 
nohistochemistry as outlined above. The only differences were the exclusion of Triton- $X$ and the use of Alexa Fluor 488-conjugated donkey anti-goat IgG (Molecular Probes) and Alexa Fluor 594-conjugated donkey anti-rabbit (Molecular Probes) diluted 1:500 as secondary antibodies.

\subsection{In vitro electrophysiology and glutamate uncaging}

To locate inhibitory inputs to PV cells from LI, we combined whole-cell patch clamp with focal photolysis of caged glutamate (Godwin et al., 1997; Schubert et al., 2007; Walker et al., 2016). Animals were deeply anaesthetized with isoflurane and sacrificed by decapitation. Thalamocortical slices of $300 \mu \mathrm{m}$ thickness containing the barrel cortex were prepared with a vibratome (VT1200S; Leica, Germany). The cooled $\left(4^{\circ} \mathrm{C}\right)$ and oxygenated $(95 \% \mathrm{O} 2$ and $5 \% \mathrm{CO} 2)$ cutting solution containing the following (in $\mathrm{mM}$ ) was used: 75 sucrose, $87 \mathrm{NaCl}, 2.5 \mathrm{KCl}, 0.5 \mathrm{CaCl}, 7.0 \mathrm{MgCl} 2,26 \mathrm{NaHCO}, 1.25 \mathrm{NaH} 2 \mathrm{PO}$, and 10 glucose, $\mathrm{pH}$ 7.4. Slices were incubated in oxygenated artificial cerebrospinal fluid (ACSF) (in $\mathrm{mM}): 125 \mathrm{NaCl}, 2.5 \mathrm{KCl}, 2 \mathrm{CaCl}, 1 \mathrm{MgCl}$, $26 \mathrm{NaHCO}$, $1.25 \mathrm{NaH} 2 \mathrm{PO} 4$ and 25 glucose, $\mathrm{pH} 7.4$ ) for $0.5-1 \mathrm{~h}$ at $32^{\circ} \mathrm{C}$ and kept at room temperature until further processing. Slices were transferred to a fixed-stage recording chamber in an upright microscope (Axio Examiner, Zeiss, Germany) and continuously perfused at a rate of $2 \mathrm{ml} / \mathrm{min}$ at $32{ }^{\circ} \mathrm{C}$ with ACSF. Whole-cell patch-clamp recordings from $\mathrm{PV}$ interneurons in LII/III, identified by tdTomato fluorescence, of the barrel cortex were performed in current clamp as well as voltage clamp. Filamented borosilicate glass capillaries (Science Products, Germany) of 5-8 $\mathrm{M} \Omega$ resistance were filled with a cesium-based internal solution (in $\mathrm{mM}$ ): 135 CsMeSO4, 5 CsCl, 0.5 EGTA, 10 HEPES, 4 Mg-ATP, 0.3 Na-GTP, 10 Na-phosphocreatine phosphate; $\mathrm{pH}$ : 7.4 and $0.3-0.5 \%$ biocytin.

For photostimulation, a 405nm diode laser (DL-405, Rapp OptoElectronic, Wedel, Germany) was coupled via a $200 \mu \mathrm{m}$ liquid fibre to the epifluorescence path of the microscope and guided into the $40 \mathrm{x}$ water immersion objective (Olympus, Germany). The laser beam was focused on a $50 \times 50 \mu^{2}$ area on the plane of the brain slice. Caged glutamate (CNB-caged-L-glutamate, Molecular Probes, Carlsbad, USA) was added to the ACSF perfusion with a final concentration of $330 \mu \mathrm{M}$. To reduce detection errors of IPSCs, laser stimulus ( $6 \mathrm{~ms}$ duration) was repeated three times per field at an interval of $10 \mathrm{~s}$.

In principle, glutamate release could activate all types of neurons, which could lead to disynaptically evoked inhibitory inputs. In a previous study in our laboratory a series of calibration experiments was set up to determine an energy level at which the laser, with its beam centered on the soma, generated spikes in the recorded cell (Walker et al. 2016). Here, we performed additional current clamp recordings of $\mathrm{LI}$ neurons in each slice, with potassium-based internal solution (in mmol: $135 \mathrm{~K}$-gluconate, $5 \mathrm{KCl}, 0.5$ EGTA, 10 HEPES, $4 \mathrm{Mg}$-ATP, $0.3 \mathrm{Na}$-GTP, $10 \mathrm{Na}$-phosphocreatine phosphate; pH: 7.4 ) and induced spiking by glutamate uncaging. A laser energy level of $\sim 120 \mu \mathrm{J}$ triggered spikes in all recorded LI neurons, which is the same level as for other interneurons in the barrel cortex (Walker et al. 2016). In subsequent mapping experiments, the PV cells were held at $+10 \mathrm{mV}$ and IPSCs were only accepted as stimulus-evoked if their amplitude exceeded the mean baseline $\pm 3 S D$, they were detected in at least two out of three stimulus repetitions and they appeared within a 10-20 ms time window after stimulus offset. The laser was moved from pia to LIV over an area covering three adjacent barrel-related columns (the middle one containing the recorded PV cell). Scanning was carried 
out systematically along rows with alternating directions (50 $\mu \mathrm{m}$ per step) controlled by the Morgentau M1 software (Morgentau Solutions $\mathrm{GmbH}$, Germany). Thus up to 162 different fields were stimulated without any intermittent gaps. Layer and column borders were estimated from the brightfield overview picture $(2.5 \mathrm{x})$ and aligned with the scanned cortical area.

Inhibitory maps were created, representing the average IPSC amplitude in fields containing sources of presynaptic inhibitory cells for the recorded LII/III PV cell. These maps were then converted into binary ones and aligned in relation to the layer borders and horizontal position of the recorded cell. From the overlay of all individual binary maps we calculated an average map depicting the confidence level (" 1 " responses in all individual maps, " 0 " no responses in all individual maps) for the position of inhibitory fields only in LI, for better visualization. Data were acquired using a SEC-05L amplifier (NPI Electronics, Germany) in discontinuous mode with a switching frequency of $50-60 \mathrm{kHz}$. The signals were filtered at $3 \mathrm{kHz}$ and digitized at 10-25 kHz using a CED Power1401 (CED Limited, England). Data were collected, stored, and analyzed with Signal 5 software (CED Limited).

\subsection{Image acquisition and processing}

Two different microscopes were used for acquiring overviews of tissue sections. First, an upright epifluorescence microscope (Axiolmager.M2, Zeiss, Germany) with a 10x objective (NA=0.3). The system was controlled by Neurolucida software (MBF Bioscience, USA). Tiles were stitched automatically during imaging. Second, an inverted epifluorescence microscope (Axio Observer, Zeiss) with a $10 x$ objective $(N A=0.3)$. The system was controlled by Zeiss software. Tiles were stitched after imaging in ZEN Blue software (Zeiss). Injection sites were imaged on an inverted confocal microscope (TCS $S p 5$, Leica, Germany) at 20x magnification (NA=0.75). High resolution images to probe putative synaptic contacts between fibres and cell somata were acquired on an inverted microscope (LSM 880, Zeiss, Germany) at $63 x$ magnification $(N A=1.4)$ in Airy Scan mode. Images were processed using Fiji software (Schindelin et al., 2012), to enhance contrast and brightness.

\subsection{Quantification and statistical analysis}

Brain regions were identified using the Paxinos mouse atlas (Paxinos and Franklin, 2001) and the Allen Brain Atlas (http://mouse.brain-map.org/experiment/thumbnails/100048576?image type=atlasand) as well as based on cytoarchitectonic landmarks visible in nuclear staining and by autofluorescence. Retrogradely labeled cells on all sections spanning from Bregma +3 to $-4.5 \mathrm{~mm}$ were counted manually in Neurolucida (MBF Bioscience). Layers were identified based on (i) the different cell densities and (ii) their relative thickness as measured in published data (Lefort et al., 2009; Prönneke et al., 2015). For a layer independent analysis we marked the pial surface and the LVI - white matter boarder and divided the space in between in 20 equally sized bins. We counted the proportion of cells in each of these bins. Cell counts were exported with Neurolucida Explorer to Excel. R software was used to sort data and perform statistical test. Additional statistical analysis was carried out in Sigma Plot (Systat Software Inc, Germany). Data was tested for normality (Shapiro-Wilk test) and equal variance (Brown-Forsythe 
test). Dependent on the distribution, we used parametric or non-parametric tests as indicated. All values are given as mean \pm SD. Graphs were produced using Origin software (Origin Lab, USA). Adobe Illustrator and InDesign were used for arrangement of pictures. 


\section{RESULTS}

\subsection{Intersectional rabies virus tracing of Parvalbumin express- ing GABAergic neurons}

The main aim of this project was to study the brain-wide, afferent inputs to PV expressing GABAergic neurons in the mouse barrel cortex. We utilized novel tools for intersectional rabies virus tracing to isolate the GABAergic population of PV cells. In addition, we used anterograde tracing and electrophysiology to verify connections revealed with RV tracing.

\subsubsection{Motivation to use intersectional RV tracing}

RV tracing usually combines a modified RV, Cre-dependent helper viruses and a Cre-driver mouse line to visualize inputs to a Cre-expressing cell class (Wall et al., 2010; Wickersham et al., 2007a). The specific and exhaustive Cre-expression in the target cell type is a prerequisite for the specificity of the tracing (Wall et al., 2010). To trace the inputs to PV cells, we wanted to use the widely common PV-Cre line (Hippenmeyer et al., 2005). Our initial tracing experiments using the PV-Cre line, however, revealed an uptake of virus into pyramidal shaped neurons in LV that were negative for PV antibody (Figure 3A, B). Fluorescent in-situ hybridization for vGlut1 in the PV-Cre/tdTomato line revealed that $12.2 \pm 5.6 \%$ of Cre-expressing cells in LV were excitatory (Figure $3 \mathrm{C}, \mathrm{n}=2$ mice, 8 sections), presumably due to a low-level expression of PV (van Brederode et al., 1991). To avoid tracing partly excitatory cells we developed an intersectional approach to isolate the inhibitory population of PV cells (Fenno et al., 2014; Madisen et al., 2015). We bred Vgat-Cre/PV-Flp mice that co-express Cre and Flp in all GABAergic PV neurons. After crossing with the tdTomato reporter (Ai65) we confirmed the almost complete absence of vGlut1 signal in tdTomato positive cells in LV $(0.1 \pm 0.4 \%$; Figure $3 C)$. These results suggest that the Vgat-Cre/PV-FIp line allows to trace the GABAergic population of PV expressing cells with a very high specificity.

To use this mouse line for tracing experiments, we applied two intersectional viruses (Figure 4A, E): (1) AAV8-Con/Fon-TVA-mCherry (from here on: AAV-TVA-mCherry), expressing the cell surface receptor TVA, required for uptake of modified RV, and the red fluorophore mCherry. (2) AAV8-Con/FonoG (from here on: $A A V-o G$ ), expressing the optimized rabies glycoprotein (Kim et al., 2016), required for spread of RV to presynaptic terminals. The constructs were validated in vitro, showing that their expression depends on the recombination by both Cre and Flp (Figure 4B-D, F, G).

Two weeks after delivery of helper viruses into Vgat-Cre/PV-Flp mice, RV- $\Delta$ G-EGFP (EnvA) (from here on RV-EGFP) was injected at the same location. It was pseudotyped with the avian sarcoma leucosis virus coat protein EnvA, the ligand of TVA receptor to restrict its transduction to TVA expressing cells. Its glycoprotein was deleted from its genome and replaced with the sequence for EGFP. In cells containing AAV delivered oG, trans-complementation of oG allowed RV-EGFP to spread to first-order presynaptic cells. Because this RV expressed EGFP, the presynaptic neurons appeared green. The starter cell population from which RV had spread, appeared yellow due to the mixture of EGFP and 
mCherry (Figure 5). This system should ensure that we selectively trace the monosynaptic inputs to GABAergic PV cells.
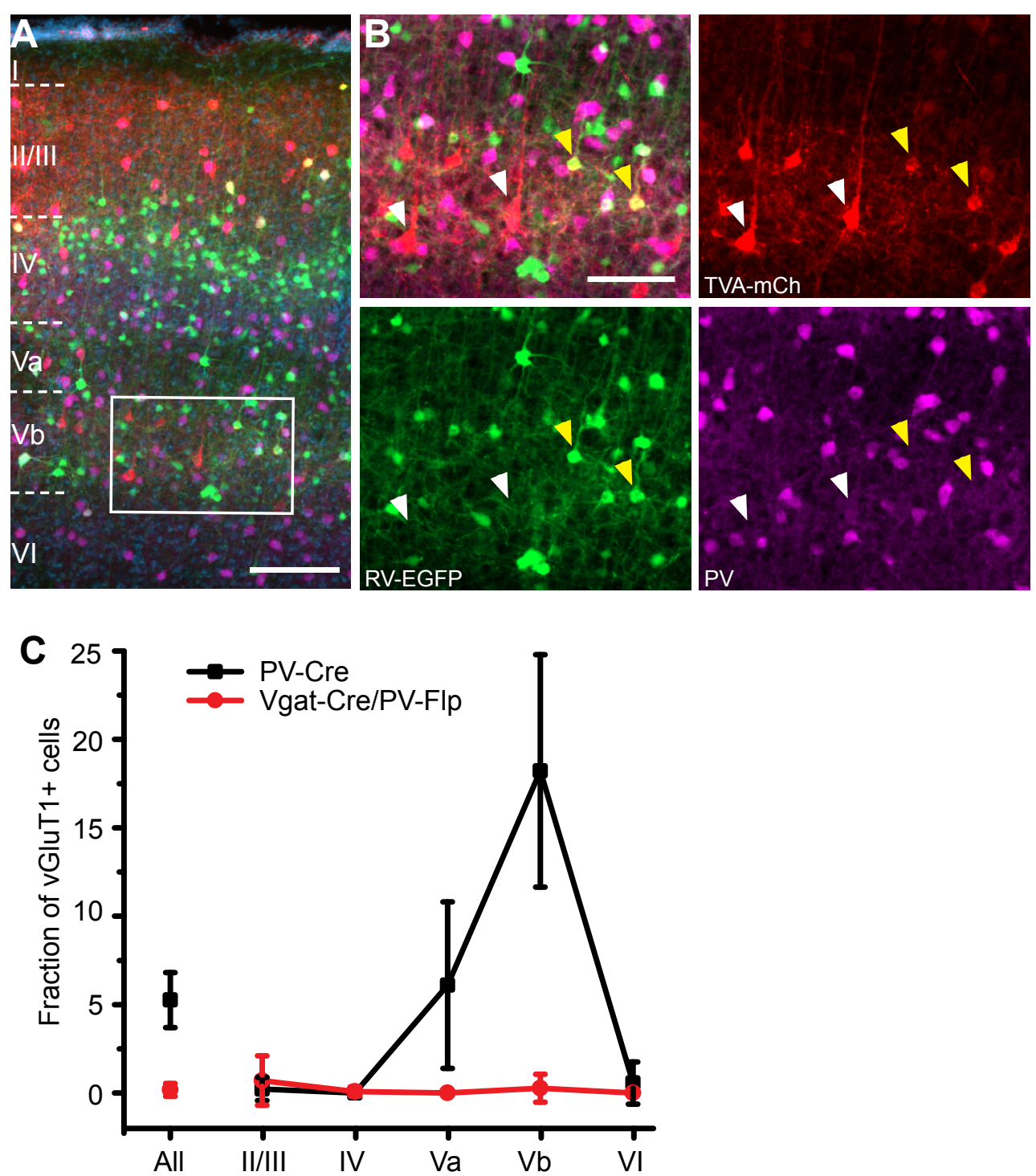

Figure 3: RV-tracing in PV-Cre line includes excitatory starter cells

(A) Injection of Cre-dependent helper viruses AAV-FLEX-TVA-mCherry and AAV-FLEX-oG, followed by RV-EGFP into PV-Cre mice revealed a reliable transduction of pyramidal-shaped neurons in LV.

(B) Insert in A. White arrowheads mark pyramidal-shaped, PV-immunonegative cells that express TVA-mCherry probably due to a low-level expression of PV protein, sufficient to activate Cre. Yellow arrowheads mark cells that additionally took up RV and are potential excitatory starter cells.

(C) Fraction of excitatory marker vGluT1-RNA-positive cells among all tdTomato-labeled cells in the PV-Cre/ tdTomato and Vgat-Cre/PV-Flp/tdTomato mouse line across layers ( $\mathrm{n}=$ each line 2 mice, 8 sections). In the PV-Cre line, about $12.2 \pm 5.6 \%$ of tdTomato-positive cells were vGluT1-positive in LV, while in the Vgat-Cre/ PV-FIp line this excitatory marker was virtually absent.

Bettina Pater collected the data for the PV-Cre line, Friedrich Krohn collected the data for the Vgat-Cre/PVFlp line. 
A
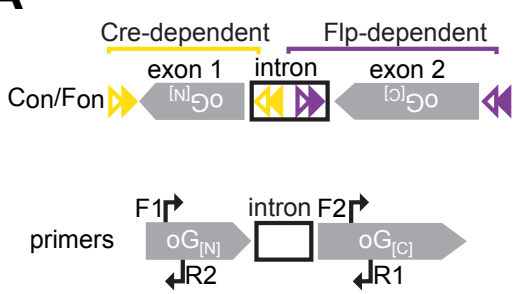

C

\section{a-Rabies Glycoprotein}

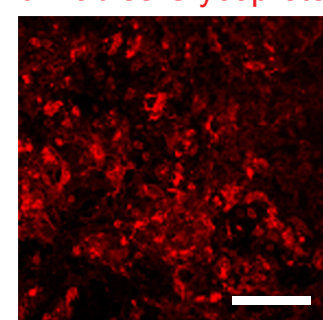

ATG OG [full length] TAA
exon 1 exon $\overline{2}$

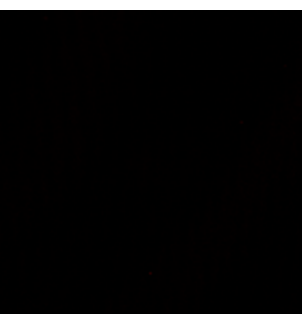

ATG oG [exon 1] TAA
B

500 bp

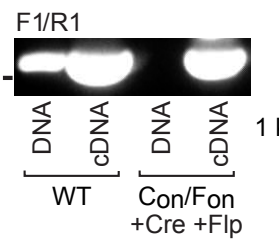

oG Map GCAGGGGC

Con/Fon-oG cDNA GCAGGGGC exon 1 /exon 2

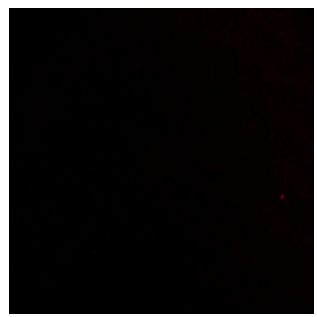

OG [exon 2] TAA
D

Con/Fon-oG
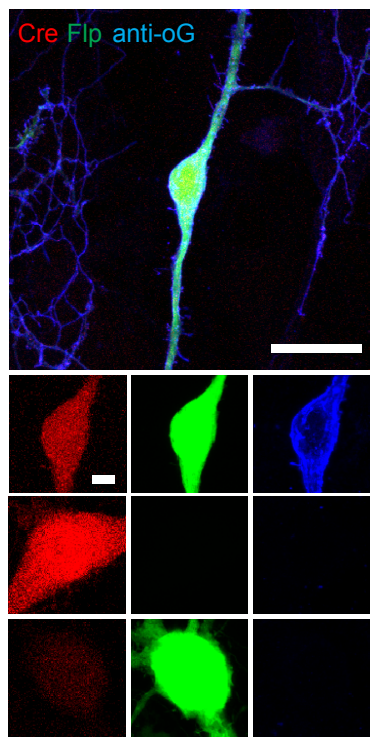

G

Con/Fon-TVA-mCherry

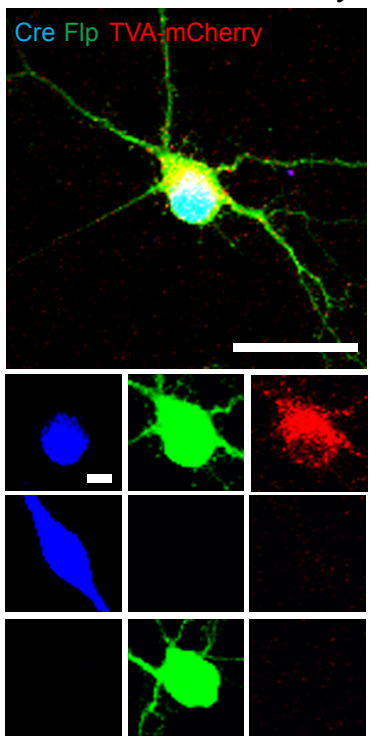

$\mathbf{F}$

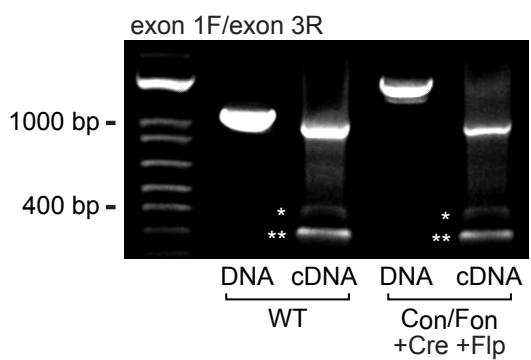

TVA-mCherry map GCAGGACT CCAGGACT

Con/Fon CDNA GCAGGACT CCAG|GACT

exon 1/exon 2 exon 2/exon 3

TVA-mCherry map ACGGGGAG TGCAGGAC

* TVA-mCherry cDNA ACGG

* Con/Fon cDNA ACGG

mid-exon 1

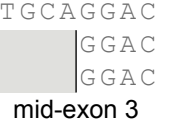

** mis-primed

Figure 4: Engineering and validation of Cre- AND Flp-dependent AAV-Con/Fon-TVA-mCherry and AAV-

\section{Con/Fon-oG}

(A) Molecular design (top) of Cre- (yellow) and Flp-dependent (purple) exons of oG (gray), created through the introduction of a central artificial intron (open box) and (bottom) primers used for PCR and RT-PCR.

(B) PCR (lanes 1 and 3) and RT-PCR (lanes 2 and 4) using noted primers of wild-type oG (left) and Con/FonoG (right), showing expected band for Con/Fon-oG confirming proper exon re-orientation after recombinase activity and intron splicing. Splicing was further validated by sequencing of the Con/Fon-oG cDNA band (bottom). PCR of Con/Fon-oG DNA using primers recognizing the exons in the initial, reverse complement orientation gives a larger band including introns (right). 
(C) Wildtype oG (left) but not fragments used for exon 1 or exon 2 (center, right) encodes functional rabies glycoprotein, as assayed by antibody staining in HEK293 cells.

(D) Con/Fon-oG only encodes functional protein in the presence of both Cre and Flp while neither Cre nor Flp in isolation is sufficient to produce functional glyco $\neg$ protein, as assayed by flow cytometry on HEK293 cells co-transfected with Con/Fon-oG and indicated recombinases (scale bars: big panel: $50 \mu \mathrm{m}$, small panels: 5 $\mu \mathrm{m})$.

(E) Molecular design (top) of Cre- (yellow) and Flp-dependent (purple) exons of TVA-mCherry created through the introduction of two introns (open boxes) and (bottom) primers used for PCR and RT-PCR.

(F) PCR (lanes 1 and 3) and RT-PCR (lanes 2 and 4) using noted primers of wild-type TVA-mCherry (left) and Con/Fon-TVA-mCherry (right), showing expected band for Con/Fon-TVA-mCherry cDNA and confirming proper exon re-orientation after recombinase activity and intron splicing. Splicing was further validated by sequencing of the WT- and Con/Fon-TVA-mCherry cDNA bands, including minor ones (bottom). The major band represents ideal splicing of the intron and recombinase components, while the smaller bands in both the wild-type and INTRSECT versions are either non-specific or represent inherent splicing separate from the introns introduced during INTRSECT molecular engineering.

(G) Cultured neurons express functional Con/Fon-TVA-mCherry (red) only when co-transfected with Cre (blue) and Flp (green) (scale bars: big panel: $50 \mu \mathrm{m}$, small panels: $5 \mu \mathrm{m}$ ).

The figure was made by Lief Fenno. The data shown in this figure as well as the viral constructs were generated by Lief Fenno, Charu Ramakrishnan, Yoon S. Kim and Karl Deisseroth and shared with us in the framework of a collaboration.

\subsubsection{Targeting of the $\mathrm{C} 2$ column}

We directed all our virus injections to the barrel cortex, the vibrissal area of the somatosensory cortex. Each whisker has a corresponding columnar representation in the barrel cortex but the cytoarchitecture of each whisker related column is unique and slightly different from each other (Meyer et al., 2013). To achieve a highly homogenous population of PV starter cells that is well comparable among animals, we centered our injection on the C2 column, which we targeted using ISOI (Grinvald et al., 1986; Guy et al., 2015). Stimulation of the C2 whisker elicited a highly localized hemodynamic signal at the corresponding location of the $\mathrm{C} 2$ column in barrel cortex, which we mapped on the blood vessel pattern (Figure 6A, B). In a proof of principle experiment we localized the C2 column in a Scnn1a-Cre/ tdTomato mouse, which labels LIV cells of barrel cortex with tdTomato. The injection with DiO was well targeted towards the $\mathrm{C} 2$ barrel (Figure $6 \mathrm{C}$ ). For virus injections, we did not attempt to restrict out starter cell population to just one column but intended to center it on $\mathrm{C} 2$.

\subsubsection{Control experiments reveal a leak of "invisible" TVA}

To verify that not just our mouse line but also our viruses have high specificity, we performed several important control experiments. First, we injected RV-EGFP without prior injection of the AAVs ( $n=4$ hemispheres) to verify that the transduction of RV-EGFP exclusively depends on the interaction with TVA. We did not detect EGFP labeled cells indicating that RV-EGFP failed to transduce TVA-negative cells (Figure 7A). Because we made sure that this particular batch of RV-EGFP was not contaminated 


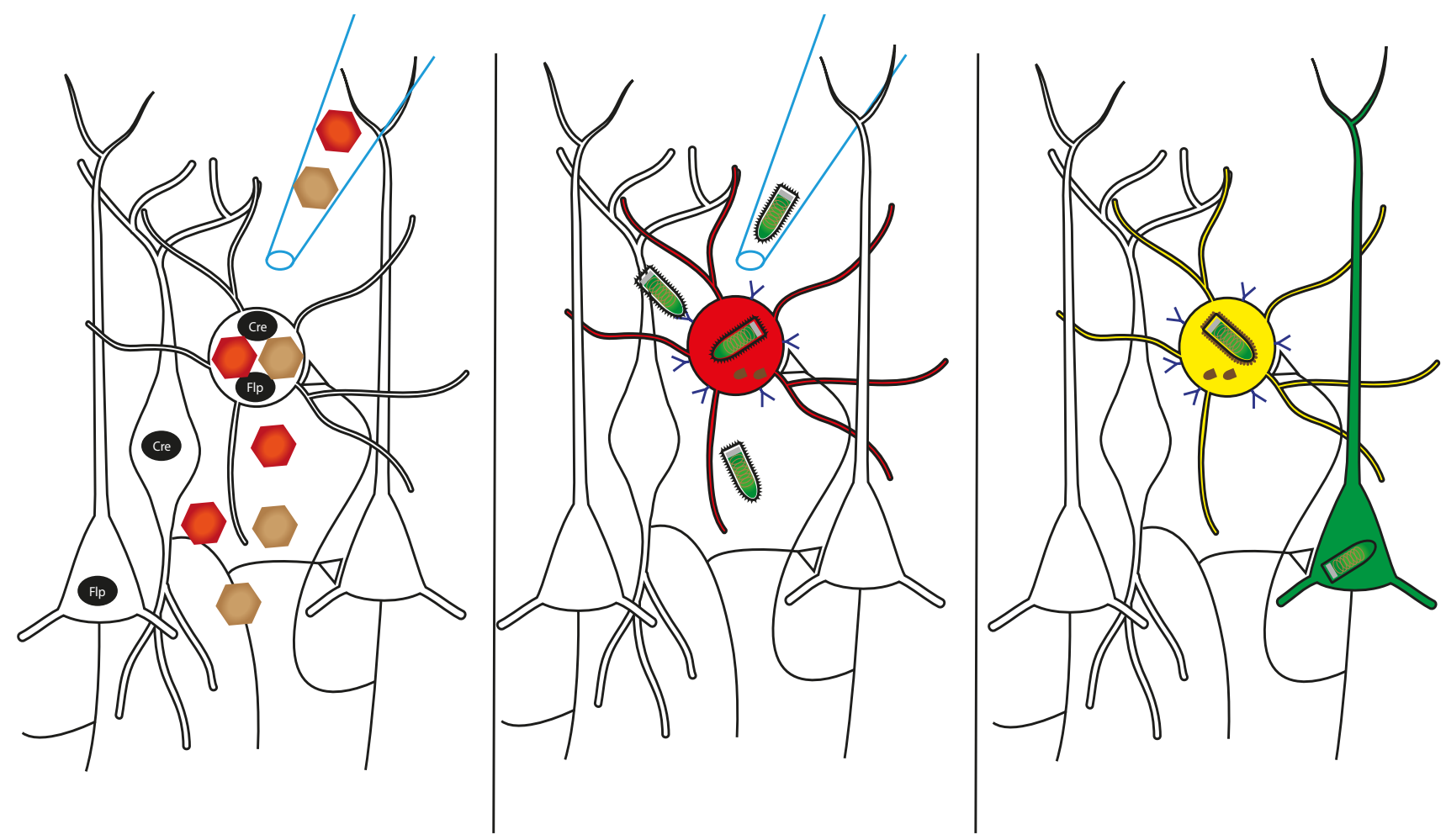

AAV8-Con/Fon-TVA-mCherry

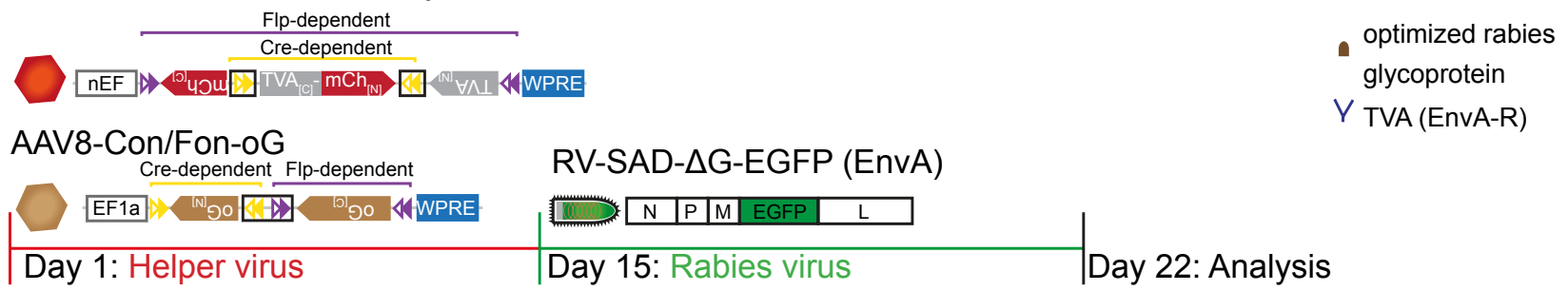

Figure 5: Experimental workflow of intersectional RV-tracing of GABAergic PV cells

On day 1, AAV8-Con/Fon-TVA-mCherry and AAV8-Con/Fon-oG are co-injected into the barrel cortex of VgatCre/PV-Flp mice. The expression of TVA-mCherry and $O G$ is conditional on the activity of both Cre and Flp. Cutting by recombinases prompts the correct orientation of the constructs. Subsequent splicing of artificial introns (black boxes) enables the assembly of the constructs. Because in Vgat-Cre/PV-Flp mice co-expression of Cre and Flp is exclusive to the intersectional population of GABAergic PV cells, TVA-mCherry and oG expression is restricted to this cell-type.

RV-SAD- $\triangle$ G-EGFP (EnvA) (RV-EGFP) is injected 14 days later at the same location. EnvA protein conditions RV transduction to TVA-exposing cells. Upon uptake into GABAergic PV cells, it incorporates the oG into its envelope, which allows the spread to first-order presynaptic neurons. The absence of a glycoprotein in these neurons prohibits further spread. RV-EGFP expression mixes with AAV-mCherry expression to label starter cells yellow, while the presynaptic neurons exclusively express RV-EGFP and thus appear green.

with RV particles coated by the endogenous glycoprotein, we used only this batch for subsequent experiments.

Second, we investigated if the recombination of the AAVs only happens in the presence of both recombinases. We injected BL6 mice, which express neither Cre nor Flp, with experimental titers of AAV-TVA-mCherry and AAV-oG before injection of RV-EGFP ( $n=2$ mice). We observed weakly mCherry-positive cells as well as a low number of EGFP-positive cells at the injection site (Figure 7B). This was also the case when we injected the same virus combination in either Vgat-Cre ( $\mathrm{n}=2$ mice) or PV- 

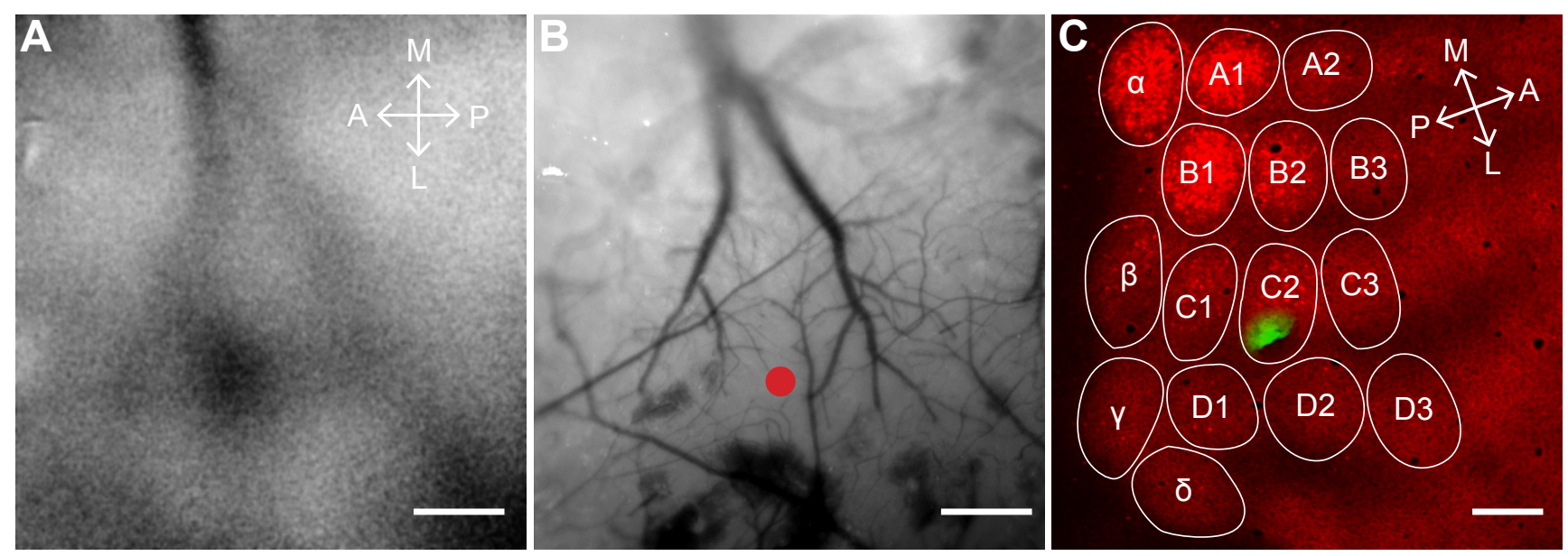

Figure 6: Mapping the $\mathrm{C} 2$ whisker representation in barrel cortex

(A) In a proof-of-principle experiment we showed that intrinsic signal optical imaging can be used to target injections to a defined column of the barrel cortex. We illuminated the exposed cortical surface above the barrel cortex with red light, while stimulating the $\mathrm{C} 2$ whisker and recording the reflectance with a CCD-camera. 30 frames were recorded and averaged. Repetitive whisker stimulation led to a localized change in blood flow, which induced a change in light reflectance visible as a dark dot.

(B) Surface vasculature was overlaid with image $A$ and the location of the highest change in reflectance was marked with a red dot. The blood vessels were used as landmarks to guide the injection pipette.

(C) Tangential section through the barrel cortex of a Scnn1a-Tge-Cre/tdTomato mouse. Injection of DiO crystals was guided by the intrinsic signal elicited upon $\mathrm{C} 2$ whisker stimulation shown in A. This experiment demonstrated the accuracy of the injection procedure (scale bars: $200 \mu \mathrm{m}$ ).

Flp ( $n=2$ mice) mice, where one recombinase was present, except that the number of EGFP-positive cells was slightly higher (Figure 7C, D). However, we never observed EGFP-positive cells outside of the injection site in any mouse line. These results lead us to the following considerations:

(I) TVA-mCherry can be expressed in the absence of one or two recombinases at a very low level. However, when we injected AAV-TVA-mCherry into Vgat-Cre/PV-Flp animals, we found that about 97\% ( $n=4$ mice, 11 sections) of TVA-mCherry positive cells were co-labeled with PV-immunostaining, indicating a high specificity of the virus (Figure $7 \mathrm{E}$ ). We do not have a clear explanation for this discrepancy but assume that under our experimental conditions TVA-mCherry labeling is mostly directed to PV cells.

(II) Our virally delivered oG did not have a fluorescent tag. An indicator that oG is expressed in the absence of recombinases would be transsynaptic spread of RV-EGFP. We saw EGFP-positive, mCherry-negative cells at the injection site but never outside of it. We closely looked at the ventral posteromedial nucleus of the thalamus (VPM), which provides reliable long-range input to the barrel cortex (Figure 7B-D). We never found any labeling there. The cells at the injection site could be transsynaptically labeled but then we would expect at least a few cells in distant areas as well. Instead, the restriction to the injection site could be due to a local leak of TVA (Do et al., 2016; Faget et al., 2016; Kim et al., 2018; Mijamichi et al., 2013; Schwarz et al., 2015; Watabe-Uchida et al., 2012; Weissbourd et al., 2014). EnvA-pseudotyped RV is highly sensitive to TVA (Mijamichi et al., 2013). Therefore, a small number of TVA molecules can already permit RV entry into cells. In case that TVA is expressed in a recombinase-independent way, RV-EGFP could enter these cells directly making them appear as transsynaptically labeled. 

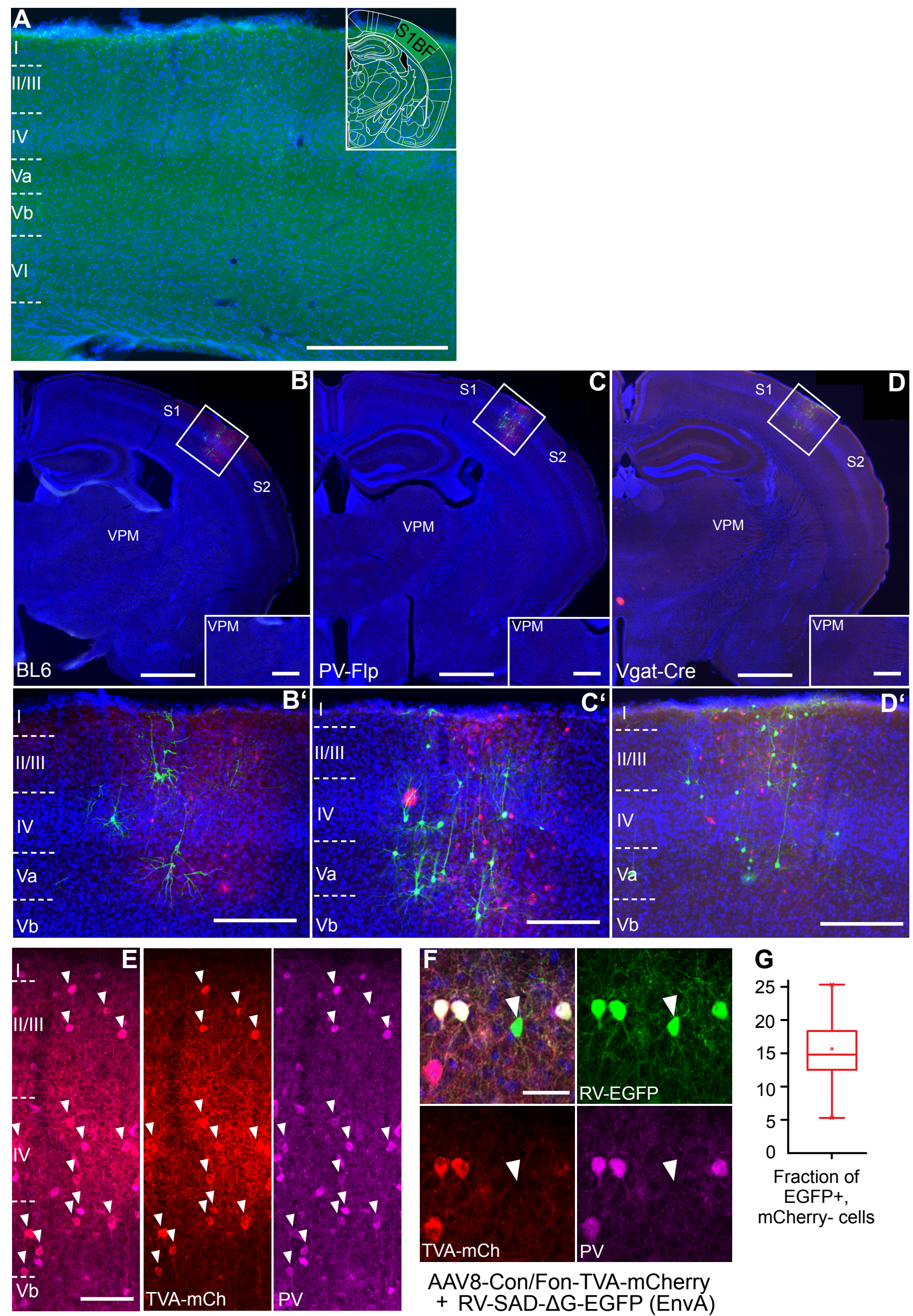


\section{Figure 7: Validating intersectional constructs for cell-type specific RV-tracing in vivo}

(A) Coronal section through the barrel cortex of a BL6 mouse after injection of RV-EGFP, without prior injection of helper AAVs. No transduced cells were detected, demonstrating that RV-EGFP transduction is TVA dependent (scale bar: $500 \mu \mathrm{m}$ ).

(B-D) Coronal sections of BL6, PV-FIp, Vgat-Cre mice after the injection of AAV-Con/Fon-TVA-mCherry and AAV-Con/Fon-oG into barrel cortex and subsequent RV-EGFP injection two weeks later. oG was not expressed, preventing RV-EGFP from spreading trans-synaptically to cells outside the injection site, as indicated by the absence of labeled cells in e.g. the ventral posteromedial nucleus of the thalamus (VPM; scale bar: overview $1000 \mu \mathrm{m}$, insert $200 \mu \mathrm{m})$.

(B'-D') Magnification of injection site. Even in the absence of both or one recombinase, TVA was expressed, allowing RV-EGFP to enter cells at the injection site directly. However, most RV-labeled cells were TVA-negative although (in principle) mCherry-expressing cells were visible. This means that RV can enter cells without a mCherry signal, most likely due to very low levels of leaky TVA (scale bar: $200 \mu \mathrm{m}$ ).

(E) Section through the barrel cortex of a Vgat-Cre/PV-FIp mouse after injection of AAV-Con/Fon-TVA-mCherry. Immunostaining for PV revealed that AAV-transduced cells virtually all co-localize with PV signal showing a high specificity of the TVA signal in this mouse line (scale bar: $100 \mu \mathrm{m}$ ).

(F) Injection of AAV-Con/Fon-TVA-mCherry alone (no AAV-oG that enables trans-synaptic spread) into a VgatCre/PV-FIp animal, followed by RV-EGFP injection two weeks later. Injections yielded mostly double-labeled, PV-positive cells, but also a few mCherry-negative, EGFP-positive cells. These exclusively green cells are a result of direct RV-EGFP entry, due to low-level expression of TVA in the absence of Cre/FIp. Because these cells do not have a mCherry signal, we termed this phenomenon "invisible TVA" (scale bar: $50 \mu \mathrm{m}$ ).

(G) On average $16 \%$ of cells ( $n=4$ hemispheres, 8 sections) at the injection site had levels of invisible TVA high enough for direct RV-EGFP entry.

(III) To investigate the extent of possible TVA leak, we injected AAV-TVA-mCherry without AAV-oG into Vgat-Cre/PV-FIp mice and two weeks later RV-EGFP ( $n=4$ hemispheres, Figure 7F). About 15.7 $\pm 6.1 \%$ of the transduced cells were exclusively EGFP-positive, mCherry-negative cells (Figure 7G). Because no oG was provided, RV must have transduced them directly due to leaky expression of TVA. These cells displayed no mCherry signal making the TVA "invisible". This suggests that RV-EGFP-positive, mCherry-negative cells at the injection site in our control experiments result most likely from direct RV uptake. Therefore, recombinase-independent expression of "invisible" TVA and not oG is responsible for the exclusively green cells at the injection site in our control experiments.

In sum, these control experiments indicate that our intersectional viruses allow the specific tracing of long-range projections to GABAergic PV cells. Tracing of local circuits is slightly confounded because transsynaptically labeled, RV-EGFP positive cells are indistinguishable from cells transduced by RV-EGFP directly due to "invisible" TVA leak.

\subsubsection{Starter cells are PV-positive and occur in layers II-VI}

Vgat-Cre/PV-Flp mice $(n=13)$ were injected with a 1:1 ratio of AAV-TVA-mCherry and AAV-oG in the $\mathrm{C} 2$ column of the right barrel cortex and two weeks later with RV-EGFP at the same location. After one 
more week, animals were sacrificed and the brain sectioned coronally (Figure 8A). Cells double labeled with mCherry and EGFP were considered putative starter cells. At least three sections through the injection site for each brain were subjected to PV antibody staining. Almost all double-labeled starter cells were PV positive $(96.6 \% \pm 3.89 \%$; Figure $8 \mathrm{~B})$ demonstrating a high specificity of the intersectional viruses. Starter cells were present in all cortical layers expect for $\mathrm{LI}$ (Figure 8C).

It is important to mention that we are likely to overestimate the number of starter cells for three reasons. First, because AAV-oG did not carry a fluorescent tag, we cannot say with complete certainty that all double-labeled cells contained oG. Co-injection of AAV-TVA-mCherry and AAV-Con/Fon-EGFP at equal titers yielded a co-transduction rate of just $31 \%$ ( $n=6$ sections, 1 mouse; data not shown). Second, oG needs to build up to a critical concentration to allow for the assembly of retrogradely infectious particles so that the mere presence of oG does not necessarily guarantee trans-synaptic spread (Weible et al., 2010). Third, PV cells are highly interconnected among each other (Karnani et al., 2016a; Tamás et al., 1998). Therefore, PV cells that took up TVA-mCherry can either be directly transduced by RV-EGFP or retrogradely if they connect to a real starter PV cell. Because of these confounding factors we abstained form calculating a ratio between starter cells and presynaptic cells as a means of normalization as exercised by others (Do et al., 2016).
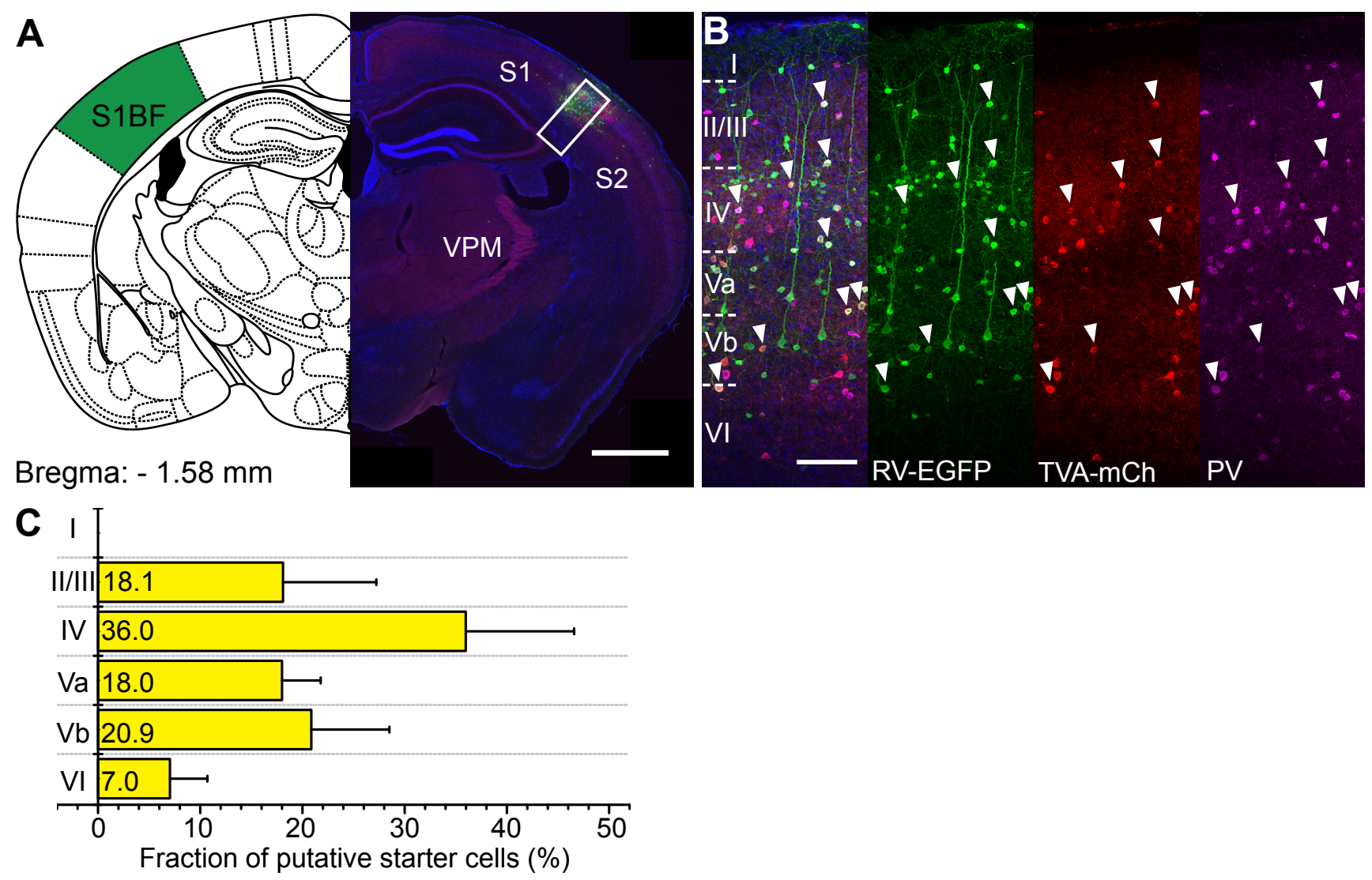

\section{Figure 8: Identification of starter cells in Vgat-Cre/PV-FIp mice}

(A) Coronal section through an injection site in right barrel cortex with the corresponding atlas section (scale bar: $1000 \mu \mathrm{m})$.

(B) Insert in A. Cells marked by white arrowheads are AAV-Con/Fon-TVA-mCherry and RV-EGFP co-transduced, putative starter cells. They are almost exclusively positive for PV protein (scale bar: $100 \mu \mathrm{m}$ ).

(C) Distribution of putative starter cells across cortical layers ( $n=13$ mice; $\pm S D$ ). 


\subsubsection{The population of PV cells receives predominantly local excitatory inputs}

We manually counted all retrogradely labeled cells on all sections throughout the rostro-caudal extent of the brain from Bregma +3 to -4.5 . Each section was overlaid with the corresponding section of the Paxinos mouse atlas (Paxinos and Franklin, 2001). EGFP positive cells were assigned to specific are-
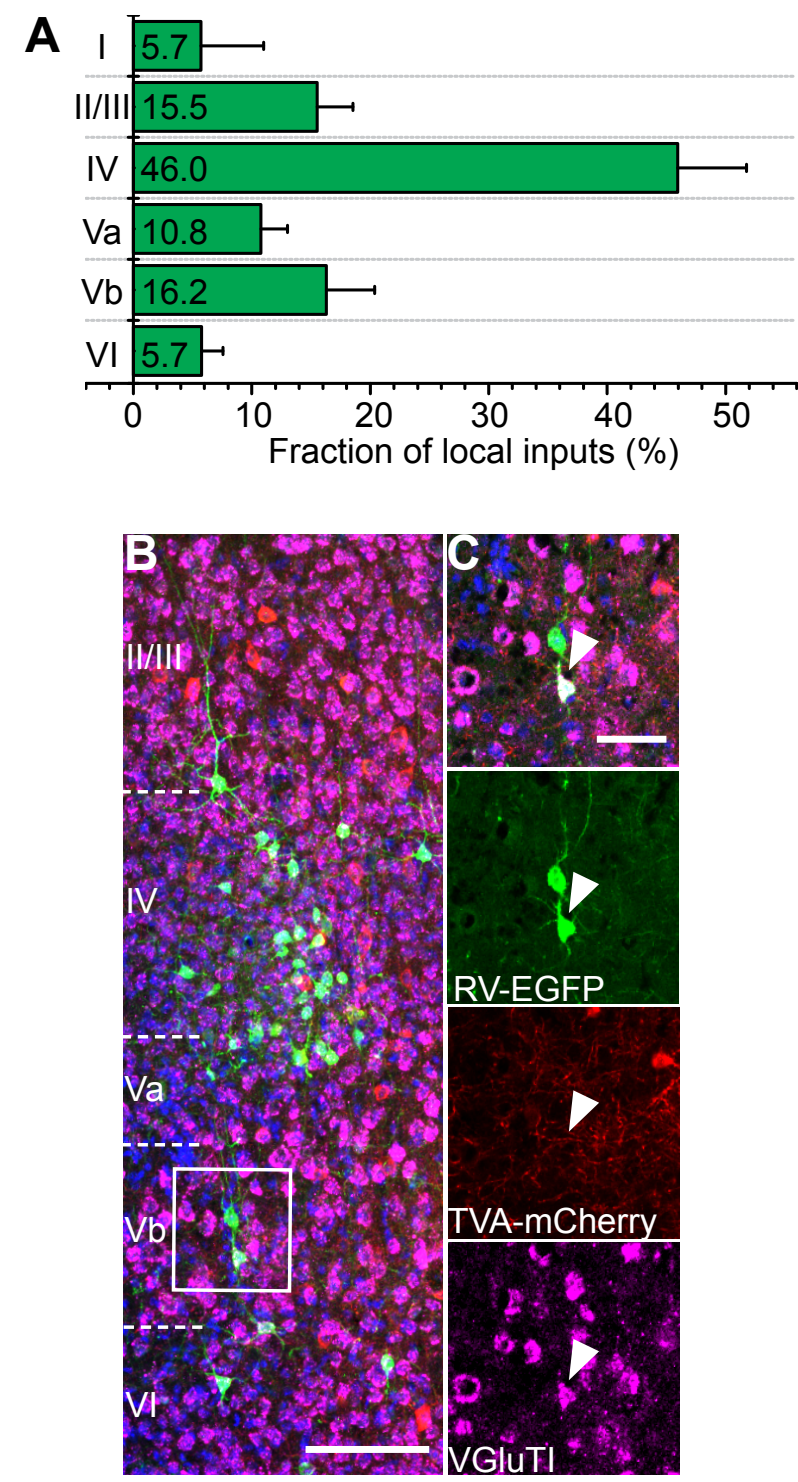

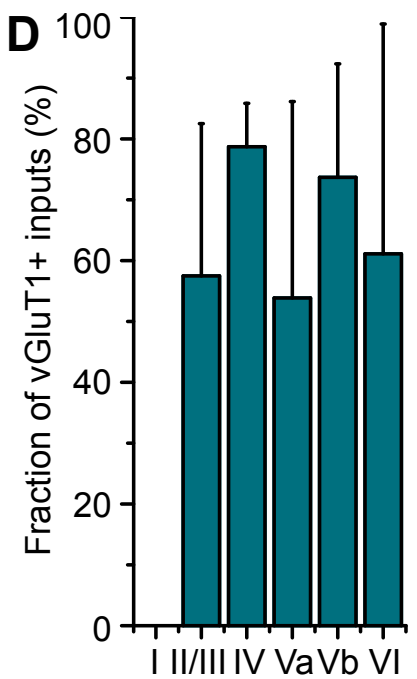

vGluT1+ inputs per layer/ vGluT1+ inputs per layer/ total inputs in layer
E

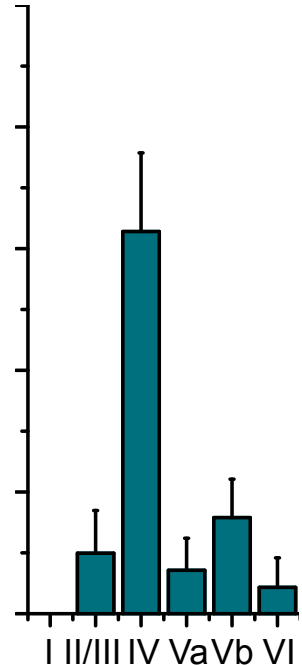

total VGluT1+ inputs
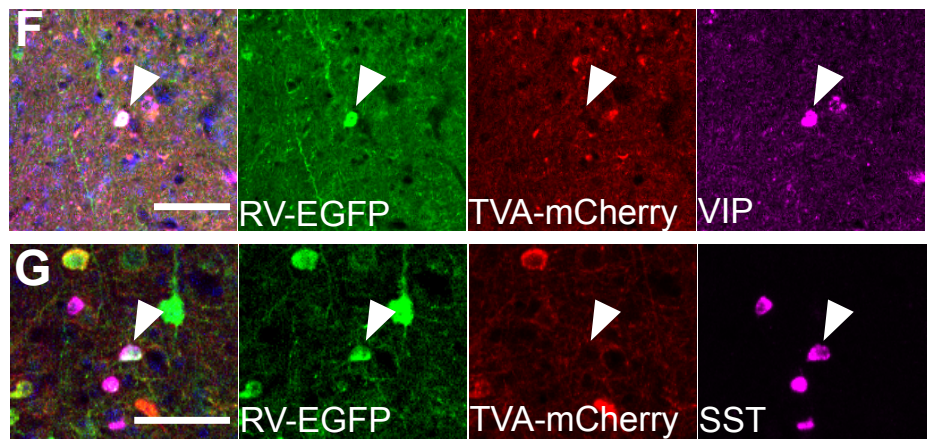

\section{Figure 9: Local inputs to PV cells}

(A) Distribution of RV-EGFP-positive cells across cortical layers (13 mice, mean \pm SD).

(B) Section through the injection site that underwent fluorescent in-situ hybridization for the excitatory neuron marker vGluT1 (scale bar: $200 \mu \mathrm{m}$ ).

(C) Insert of B. White arrowheads mark RV-EGFP-positive cells co-localizing vGluT1 probe-derived fluorescence (scale bar: $50 \mu \mathrm{m}$ ).

(D, E) Fraction of vGLUT1-positive inputs in each layer and fraction of vGluT1-positive inputs among all local inputs in each layer $(n=4$ mice, 16 sections; mean $\pm S D)$.

(F, G) Examples for RV-EGFP-positive cells co-localizing VIP and SST RNA-probe-derived fluorescence (scale bar: $50 \mu \mathrm{m})$. 
as based on the overlay and cytoarchitectonic features discernable based on DAPI counterstain.

We discriminated between local input and long-range inputs. Inputs were classified as local if they were with the barrel cortex as defined by the Paxinos mouse atlas and classified as long-range if they were outside. Local inputs made up $92.7 \% \pm 3.1 \%$ of total inputs. This dominance of local inputs suggests that the activity within the barrel cortex has much greater influence on the activity levels of PV cells than long-range inputs. We calculated the proportion of local inputs in each layer, as defined by the soma position of the presynaptic neuron (Figure 9A). Almost half of the presynaptic cells were located in LIV, where cells mostly lacked an apical dendrite suggesting them to be spiny stellate cells. It is important to keep in mind that these numbers are subject to the error caused by leak expression of TVA as mentioned earlier. However, the relative proportion of each layer should be mainly unaffected because leak expression is a random phenomenon.

To investigate the balance of excitatory and inhibitory inputs to PV cells, we performed fluorescent in-situ hybridization for vGlut1, SST and VIP on sections spanning the injection site. $70 \%$ of local inputs were positive for the excitatory marker vGlut1 (Figure 9B, C). This is also approximately the proportion of excitatory cells within each layer (Figure 9D). This means that PV neurons, as a population, receive mostly excitatory inputs. These excitatory inputs originated mostly from L4 (Figure 9E) because the largest fraction of local inputs was located in LIV. The stainings for SST and VIP yielded such low number of co-localization rates with EGFP positive cells (2.1\% for VIP, $0.7 \%$ for SST, Figure $9 F, G)$ that we abstained from a further analysis. We did not stain for GADI because PV cells itself are part of the GADI population. As RV can spread among the interconnected PV cells, starter cells and retrogradely labeled cells become intermingled. Because PV cells that appear as starter cells constitute an unknown fraction of inhibitory inputs converging on PV cells, GADI staining could not yield an unbiased value about the fraction of inhibitory inputs innervating PV cells.

\subsubsection{LI interneurons inhibit PV neurons in upper layer II/III}

Interestingly, we found that $5.7 \% \pm 5.3 \%$ of local inputs originated from LI. We performed in-situ hybridization for GADI to confirm that all LI cells were inhibitory (Figure 10A). The axonal outreach of most $\mathrm{LI}$ neurons is predominantly confined to $\mathrm{LI}$ but some cell types do extend their axon into deeper layers (Abs et al., 2018; Jiang et al., 2015; Schuman et al., 2018). We correlated the number of LI neurons with the number of PV starter cells in LII/III, LIV and LVIVI. The correlation was only significant between LI neurons and PV starter cells in LII/III (LII/III: Spearman correlation $\rho=0.81, p<0.001$; LIV: $\rho=0.297$, $p=0.31$; LV/VI: $\rho=0.54, p=0.051$ ), suggesting that LII/III PV cells are the major population presynaptic to $\mathrm{LI}$ neurons (Figure 10B). We performed glutamate uncaging experiments to provide independent evidence for a direct connection of LI interneurons to PV cells in LII/III. We produced thalamocortical slices through barrel cortex of mice in which PV cells were labeled with tdTomato. First, we recorded from LI neurons and activated them with photolysis of caged glutamate. This allowed us to calibrate the laser power to a level at which $\mathrm{LI}$ interneurons would be activated. The laser power was in a range that also activated other inhibitory neurons (Walker et al., 2016). Then we recorded from single PV cells throughout the depth of LII/II, while laser stimulating cells in LI-LIV. 6/10 PV cells showed inhibitory synaptic responses upon stimulation of LI. An activation map of an example cell is depicted in Figure 
10C, D. We averaged the activation maps of single cells and found a reliable probability of LI input to LII/II PV cells (Figure 10E). These experiments demonstrate a functional connection between LII/III PV cells and LI inhibitory neurons, revealing another disinhibitory motif in the barrel cortex.
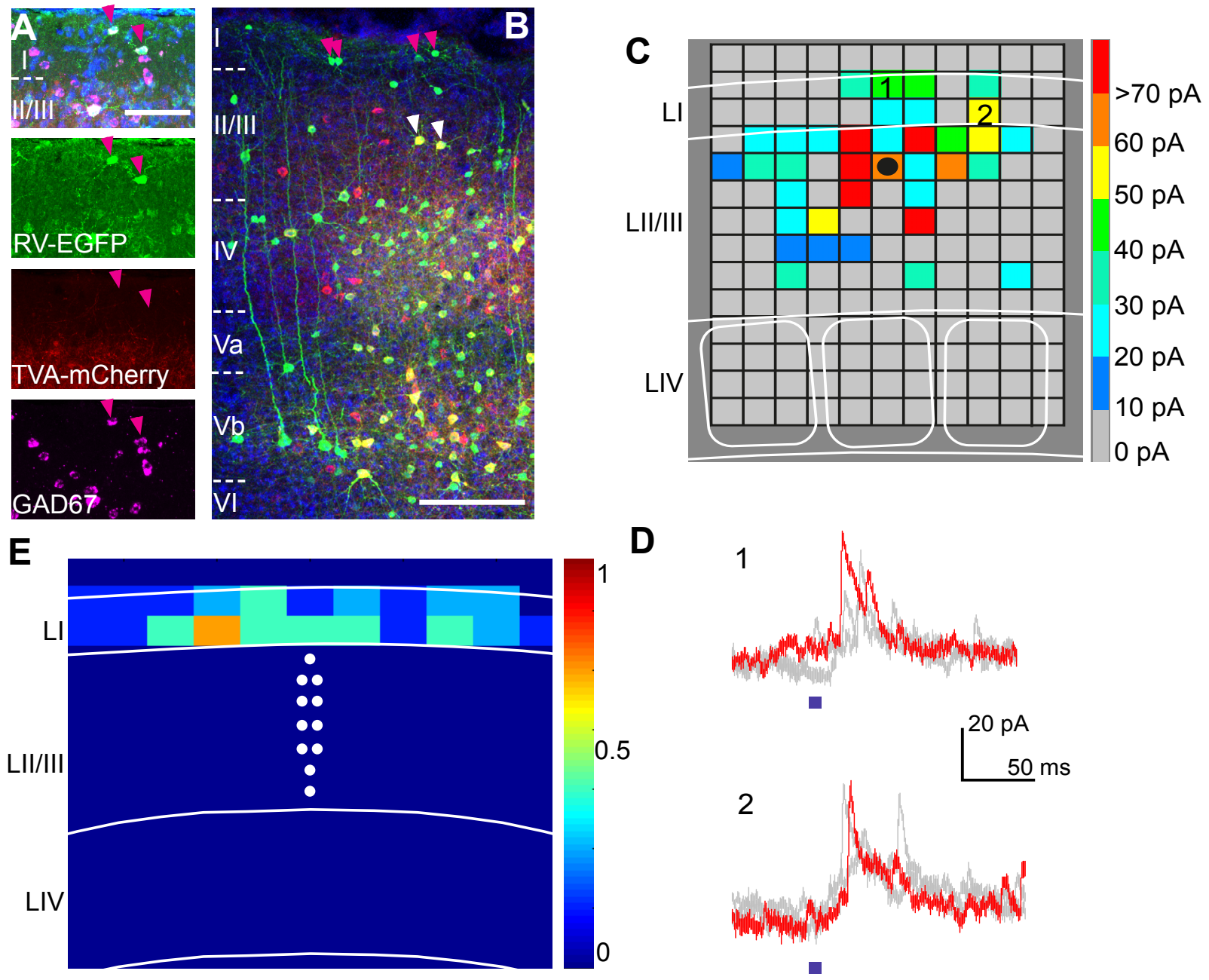

Figure 10: LI interneurons provide input to LII/III PV cells

(A) RV-EGFP-labeled cells in LI at the injection site are all positive for GAD1 mRNA, as demonstrated with fluorescent in-situ hybridization ( $n=4$ mice, 15 sections). Red arrowheads mark co-localized fluorescence (scalebar: $50 \mu \mathrm{m}$ ).

(B) Cross-section through the injection site. Red arrowheads mark RV-EGFP-positive cells in LI, while white arrowheads mark starter cells in LII/III that could potentially be postsynaptic to LI neurons (scalebar: 200 $\mu \mathrm{m})$.

(C) Example map of synaptic responses in a whole cell-recorded LII/III PV cell caused by glutamate uncaging. The color code represents the strength of the monosynaptic inhibitory postsynaptic response. Soma position is indicated by a black circle.

(D) Inhibitory postsynaptic responses recorded after photostimulation at the two squares indicated on map in C. Each field was stimulated three times. One trace is highlighted in red the other two in gray. The blue square indicates the time of laser stimulation onset.

(E) Average map ( $n=10$ cells) of postsynaptic responses onto PV cells elicited upon photostimulation in LI. White circles mark the PV neurons' soma positions across the depth of LII/III.

The electrophysiological data shown in this figure were generated by Nidhi Subhashini and analyzed with scripts written by Martin Möck. 


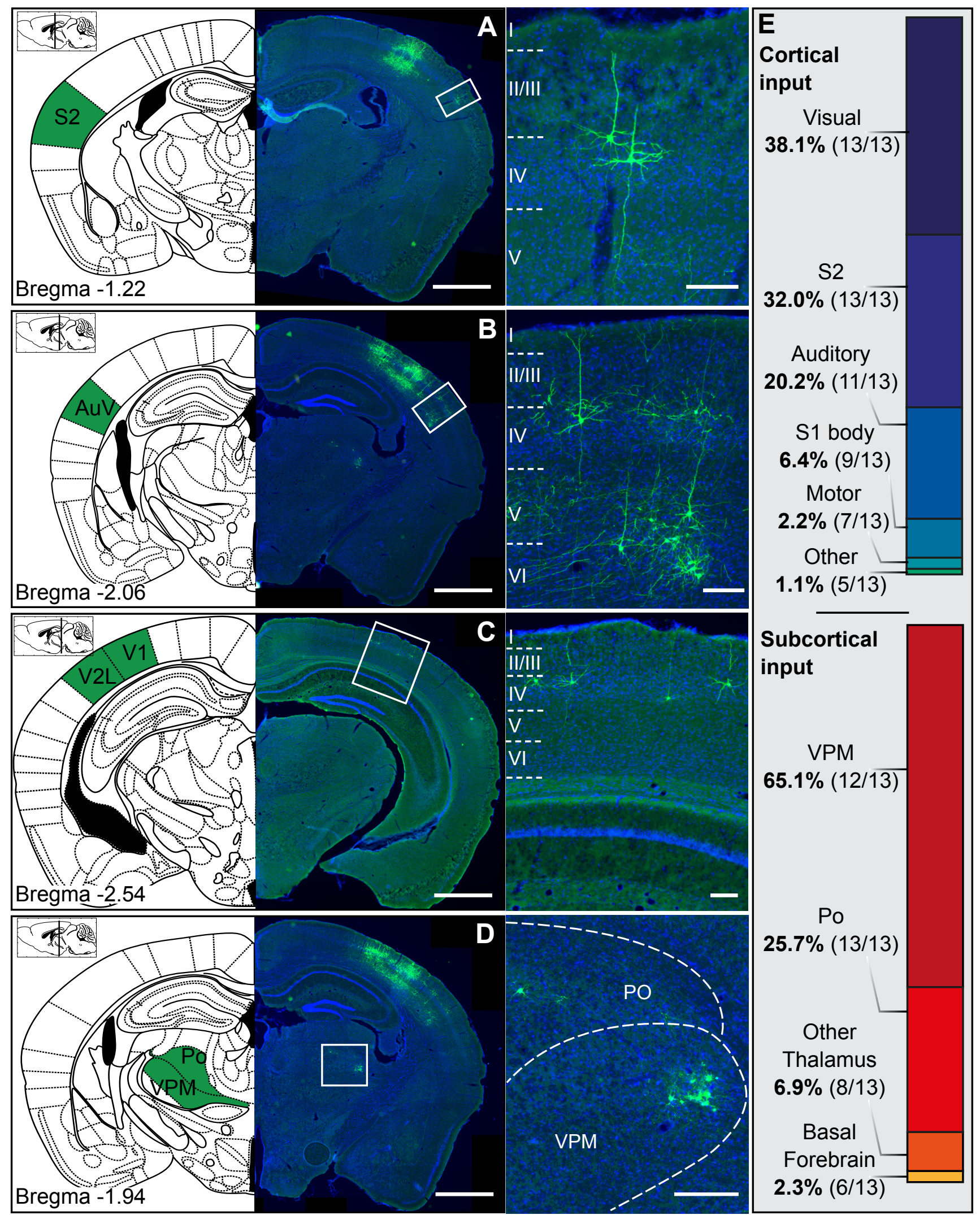

Figure 11: Long-range inputs to PV cells

(A-D) Images with corresponding Paxinos mouse atlas section showing the location of long-range inputs to PV cells in barrel cortex. The schematic sagittal section in the top left corner marks the distance from bregma. Examples are from secondary somatosensory cortex (S2; A), secondary auditory cortex (ventral area, AuV; B), primary and secondary visual cortex (V1, V2L; C), and ventral posteromedial nucleus and posterior nucleus of the thalamus (VPM, PO; D). Cortical neurons predominantly had a pyramidal morphology, suggesting an excitatory nature of the cortical long-range inputs (scalebar overview: $1000 \mu \mathrm{m}$; insert: $200 \mu \mathrm{m}$ ). 
(E) Proportion of cortical and subcortical long-range input areas. Cells in areas of similar functionality (i.e. primary and secondary visual cortex) were pooled together. Numbers in brackets indicate in how many brains (from the total sample of $n=13$ ) this area contained labeled cells.

\subsubsection{PV cells receive long-range cortical and subcortical input}

Next, we analyzed the fraction of long-range inputs originating from outside the barrel cortex. The main sources of long-range input were visual cortex, secondary somatosensory cortex, auditory cortex and the thalamus (Figure 11A-D). For quantification, we distinguished cortical $(65.1 \pm 12.7 \%$ of total longrange inputs) and subcortical (34. $9 \pm 12.71 \%$ of total long-range inputs). We calculated the percentage of inputs from each area as a fraction of the total cortical or subcortical inputs, respectively (Figure $11 \mathrm{E})$. The most numerous cortical input stemmed from visual areas, which made up almost $40 \%$ of all cortical inputs. The dominant subcortical input was with approximately $65 \%$ the ventral posteromedial nucleus of the thalamus (VPM).

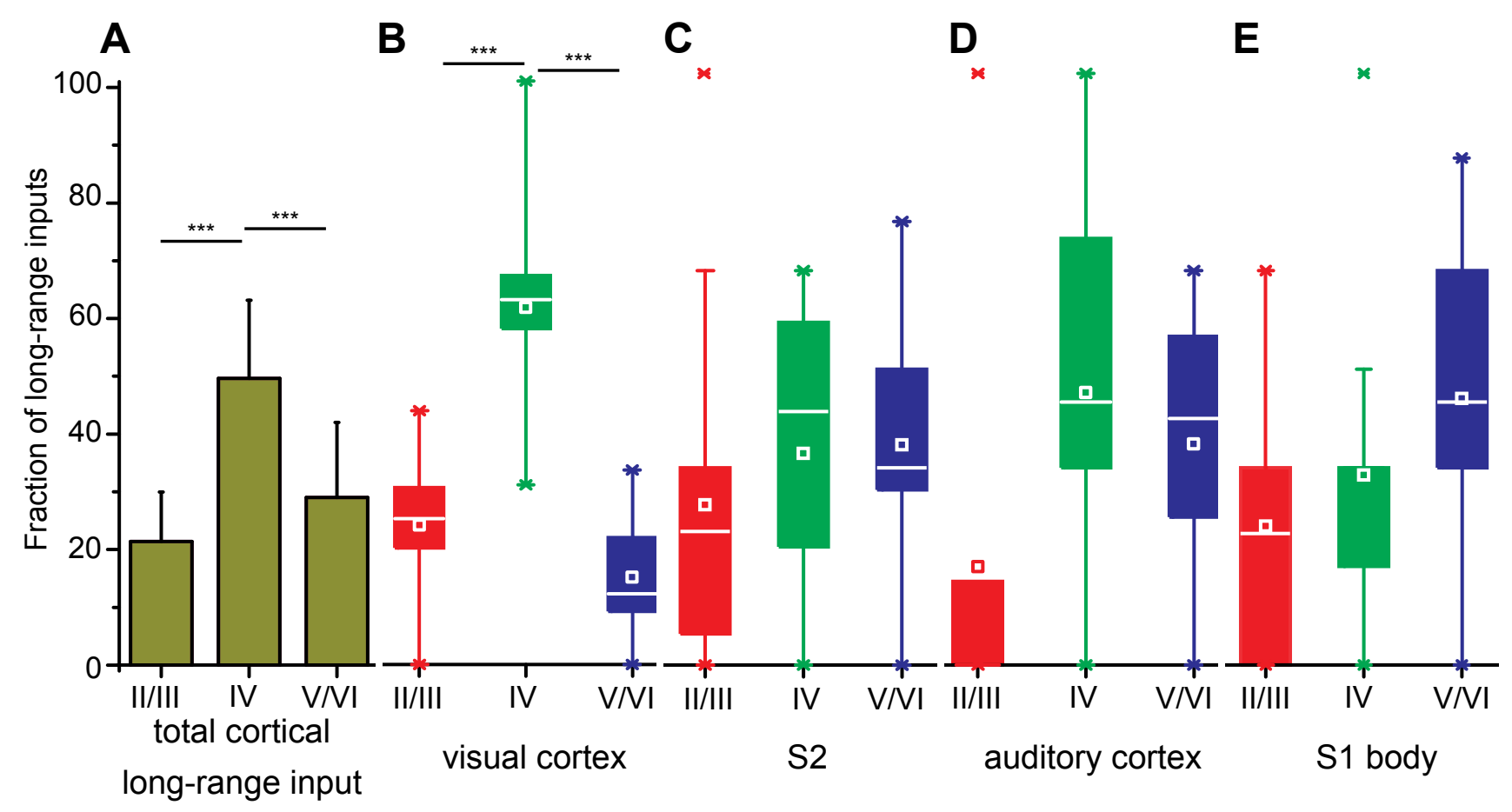

Figure 12: Laminar analysis of cortical long-range inputs to PV cells

(A) Fraction of long-range inputs by layer compartment from all cortical areas, except motor cortex (ANOVA, $F=19.625, p<0.001$, post-hoc Tukey analysis; mean \pm SD).

(B-E) Fraction of long-range inputs by layer compartment from visual cortex, secondary somatosensory cortex (S2), auditory cortex, and primary somatosensory cortex (S1) body representation. For visual cortex there was a significant difference among layer compartments, with LIV containing the highest number of labeled projection neurons (ANOVA, $\mathrm{F}=34.84, \mathrm{p}<0.001$, post-hoc Tukey analysis). $* * * p<0.001$ 

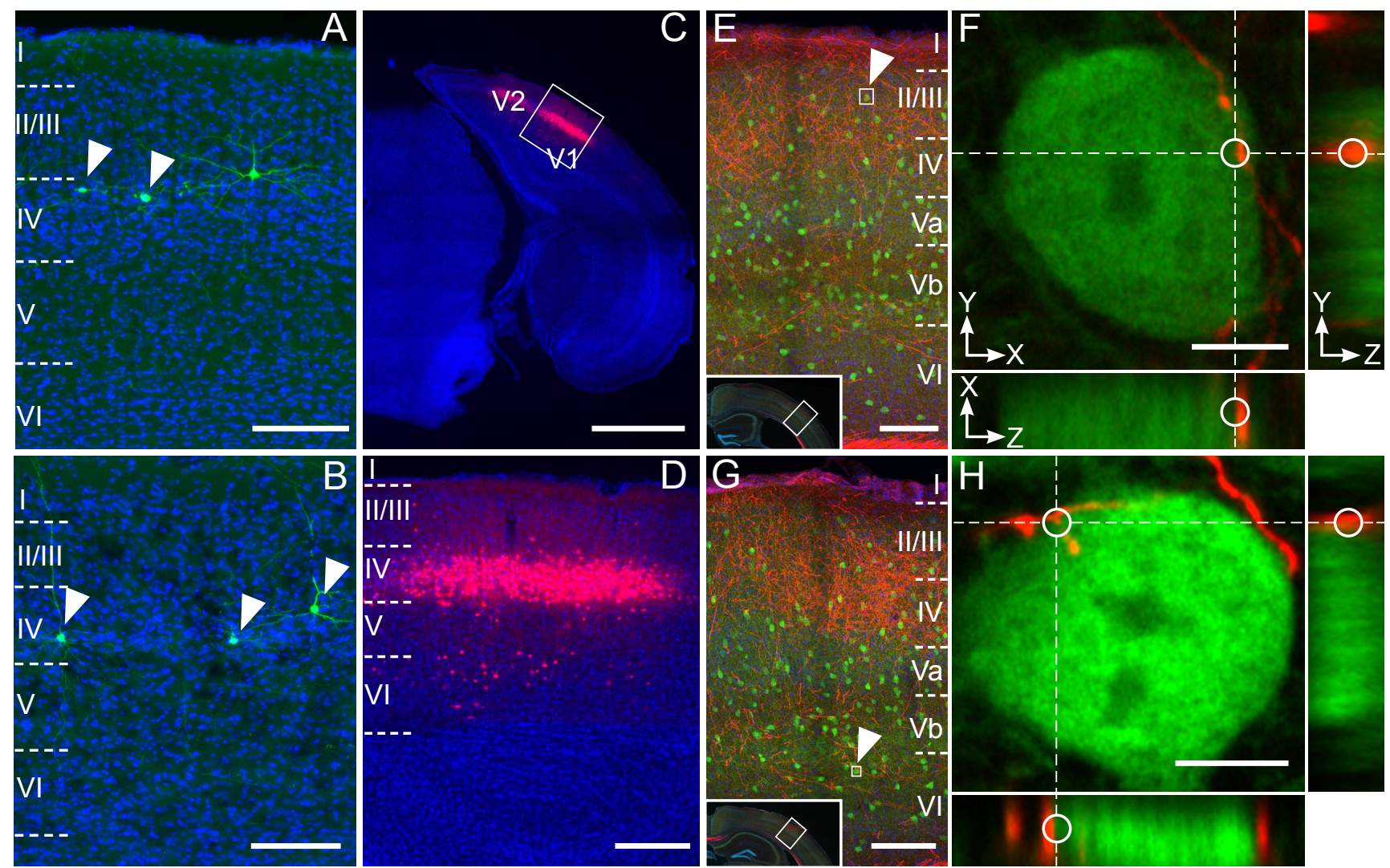

Figure 13: LIV neurons in visual cortex form putative synapses with PV cells in barrel cortex

(A, B) Additional examples for retrogradely labeled cells in visual cortex LIV (white arrowheads), which were often located at the border between LIII and LIV (scale bar: $200 \mu \mathrm{m}$ ).

(C) Coronal section through visual cortex of LIV-specific mouse line Scnn1a-Tg3-Cre, injected with AAV-FLEX-tdTomato (scale bar: $1000 \mu \mathrm{m}$ ).

(D) Insert of C with close-up of injection site. LIV cells are strongly labeled but a few transduced LV/VI cells are visible, too (scale bar: $200 \mu \mathrm{m}$ ).

$(\mathbf{E}, \mathbf{G})$ Cross-sections through barrel cortex of injected animal in $C / D$, immunostained for PV in green (scale bar: $100 \mu \mathrm{m})$.

$(\mathbf{F}, \mathbf{H})$ Insert of E/G, respectively. tdTomato-positive axons originating from visual cortex were in close apposition to PV-stained cell bodies in barrel cortex. Boxes indicate the putative contact sites in the XY, XZ, and YZ planes (scale bar: $5 \mu \mathrm{m}$ ).

\subsubsection{LIV visual cortex projects to PV neurons in the barrel cortex}

We analyzed the distribution of cortical inputs across layers, excluding inputs from motor cortex because of the nearly absent LIV. We calculated the proportion of retrogradely labeled, long-range projection neurons localized in LII/III (20.9 +/-8.4\%), LIV (50.3+/-13.8\%) and LV/VI (28.8+/-13.0\%) (Figure $12 \mathrm{~A}$ ). The number of projection neurons in LIV was significantly higher than in LII/III or LV/VI (ANOVA, $F=19.625 ; p<0.001$ ). Next, we calculated the proportion of projection neurons in each layer compartment for the main input areas individually. We found no difference among the layer compartments, except for visual cortex (Figure 12B-E; visual cortex: ANOVA, F=34.84, $p<0.001$; S2: ANOVA, F=0.62, $p=0.54$; $S 1$ body: ANOVA, $F=1.59, p=0.23$; auditory cortex: Kruskal-Wallis test, $H=5.72, p=0.06$ ). Again, 
LIV in visual cortex contained significantly more projection neurons than upper or deep layers (LII/III: $23.88 \pm 12.63 \%$, LIV=59.64 $\pm 17.71 \%$, LV/VI: $16.49 \pm 11.11 \%$, Tukey Test, $p<0.001$ for both LIV vs. LII/ III and LIV vs. LV/VI).

We observed that retrogradely labeled projection neurons in visual cortex LIV were often at the border between LIII and IV opening the possibility that these are deep LIII cells (Figure 13A, B). To provide additional evidence for this unexpected connection motif from LIV visual cortex to barrel cortex, we injected an anterograde tracer AAV2/1-FLEX-tdTomato into visual cortex of LIV-specific Scnn1aTg3-Cre mice $(n=2)$ to visualize fibres from LIV cells reaching barrel cortex (Figure 13C, D). The fibre density was highest in LII/III of barrel cortex but we could find putative synaptic contacts between fibres and PV neurons throughout all layers of the barrel cortex (Figure 13E-H). This experiment adds evidence that LIV cells in visual cortex maintain long-range connections to barrel cortex, of which at least a fraction are targeting $\mathrm{PV}$ cells. 


\subsection{Comparison of brain-wide inputs to VIP neurons in barrel cortex of WT and reeler mice}

The main aim of this project was to compare the brain-wide long-range inputs to VIP neurons in WT and reeler mice. The project was motivated by the question if VIP neurons in reeler are embedded in the same long-range circuitry as in WT animals despite the absence of cortical layers. We optimized and then applied Cre-dependent RV tracing to visualize the brain-wide monosynaptic inputs to VIP neurons in both genotypes.

\subsubsection{VIP neurons are misarranged in the reeler mouse}

Cortical layers do not form in reeler (D'Arcangelo and Curran, 1998). In previous studies, staining for laminar fate markers revealed that excitatory neurons with a molecular fate for a particular layer are malpositioned (Boyle et al., 2011; Dekimoto et al., 2010; Wagener et al., 2010). On the contrary, the position of inhibitory neurons in the reeler mouse has only been minimally investigated (Boyle et al., 2011; Hevner et al., 2004; Pielecka-Fortuna et al., 2014; Yabut et al., 2007). Unlike excitatory cells,
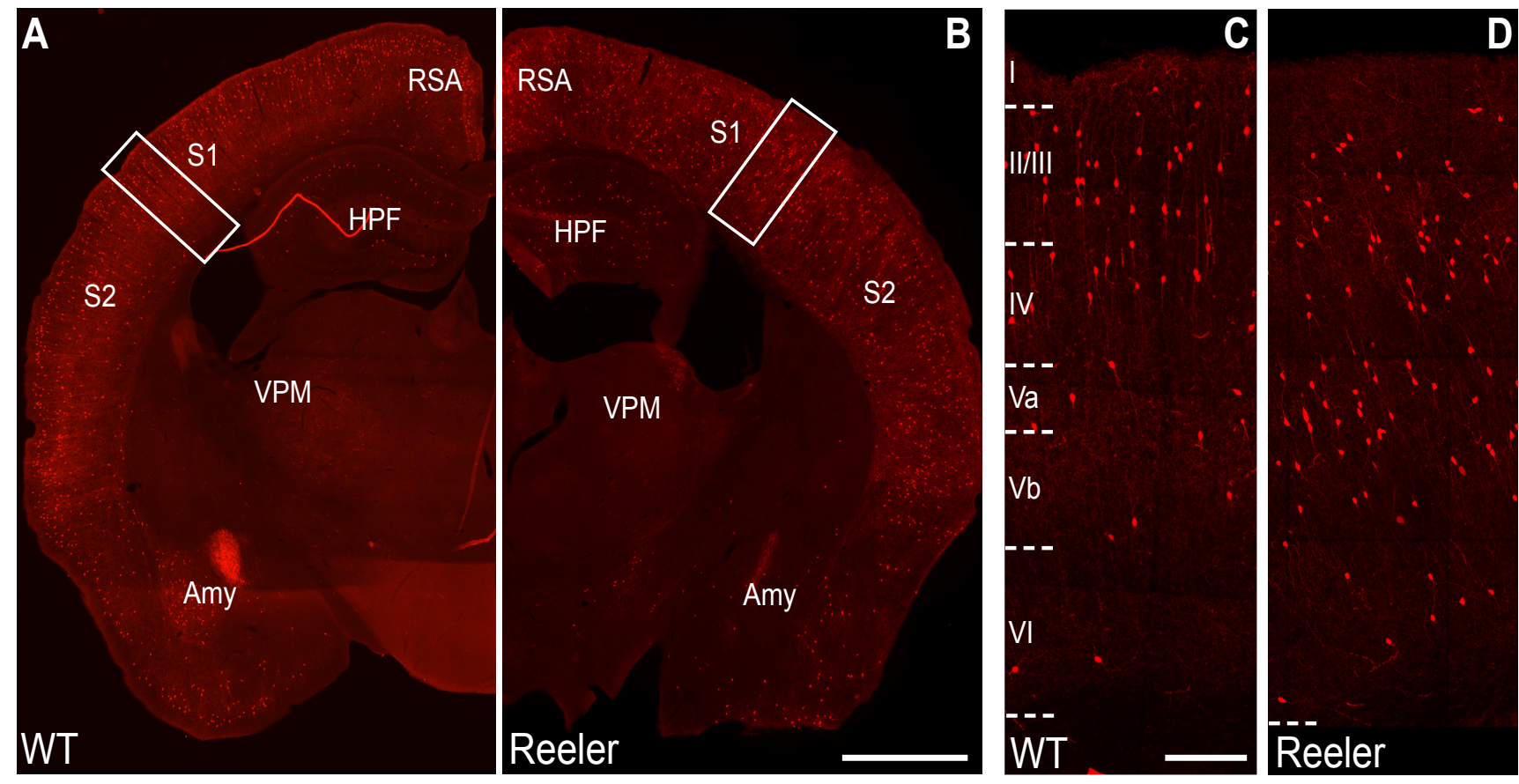

Figure 14: Distribution of VIP cells in WT and reeler mice

(A, B) Coronal section at the level of the barrel cortex of a VIP-Cre/tdTomato and VIP-Cre/tdTomato/Reeler mouse (scalebar: $1000 \mu \mathrm{m}$ ).

(C, D) Insert in A, B. Close-up of cortical tissue in WT and reeler mouse. VIP neurons labeled with tdTomato show a stronger bias towards upper layers in WT and a more dispersed arrangement in reeler (scale bar: 100 um; Abbr.: Amy, Amygdala; HPF, hippocampal formation, RSA, retrosplenial agranular cortex; S1, primary somatosensory cortex; S2, secondary somatosensory cortex).

The tissue sections were obtained from Julien Guy. 
they do not have a layer fate marker, although each interneuron subtype has a fairly distinct laminar profile in WT cortex (Hioki et al., 2013). Among the three main types of interneurons, VIP neurons have the most biased laminar distribution. About $60 \%$ of VIP neurons in barrel cortex are in LII/III (Prönneke et al., 2015). We investigated if VIP neurons in the reeler mouse have such a bias, too, or if they are arranged differently.

We generated VIP-Cre/tdTomato and VIP-Cre/tdTomato/reeler mice to visualize VIP neurons. In reeler mice, VIP neurons appear homogeneously distributed throughout the thickness of the cortex and are not biased towards a certain layer (Figure 14). Given this dispersion, we focused on the question if VIP neurons in reeler are able to integrate long-range input from the same sources and with the same magnitude.

We used Cre-dependent RV tracing to map the monosynaptic long-range inputs to VIP cells in barrel cortex of WT and reeler mice (Figure 15). We injected helper AAV vector AAV8-FLEX-TVA ${ }^{66 T}$ EGFP-oG (from hereafter AAV-TVA ${ }^{66 T}$-EGFP-oG) into the barrel cortex of VIP-Cre and VIP-Cre/reeler mice. It carries the sequence for three proteins separated by $2 A$ elements to allow for their expression in equal amounts (Minskaia et al., 2013): The cell surface receptor TVA (here we used a mutated version, TVA $^{66 T}$, which we will explain later), the optimized version of the rabies glycoprotein (oG) and EGFP as a fluorescent marker. Modified rabies virus RV- $\Delta \mathrm{G}-\mathrm{mCherry}$ (EnvA) (from hereafter RV-mCherry) was injected two weeks later at the same location. Because it is coated with EnvA, the ligand for the TVA receptor, it can only transduce cells presenting TVA on their surface. Furthermore, RV-mCherry is G-deleted, so that it needs the glycoprotein provided in trans from the helper virus to spread to presynaptic neurons. Its mCherry expression labels these presynaptic neurons red. The starter cells appear yellow due to the mixture of EGFP and mCherry. This two-virus system allows the visualization of brain-wide monosynaptic inputs to unequivocally identifiable starter cells.

\subsubsection{Mutated version of TVA abolishes leak expression of TVA}

There are two main prerequisites for a truly cell-type specific RV tracing. (1) Cre-expression must occur only and in all cells of the target cell type. (2) A solely RV positive cell must be without doubt presynaptic to a Cre-expressing cell.

Previous studies have demonstrated a very high specificity of the VIP-Cre line (Prönneke et al., 2015; Taniguchi et al., 2011). In fact, $99 \%$ of Cre-expressing cells are immunopositive for VIP and there are basically no VIP cells that do not express Cre (Prönneke et al., 2015). Thus, this mouse line fulfills the requirement for cell-type specific tracing.

To check if our viral constructs allow for cell-type specific input tracing we performed control experiments in BL6 animals that do not express any Cre. First, we injected RV-mCherry or RV-EGFP into BL6 animals without prior injection of helper AAVs. We did not see any mCherry or EGFP positive cells, respectively, at the injection site indicating that RV failed to transduce cells in the absence of TVA (Figure 16A, B).

Next we tested different AAV constructs. We injected the AAVs into the barrel cortex of BL6 animals and two weeks later RV at the same location. After termination of the experiments we looked for 


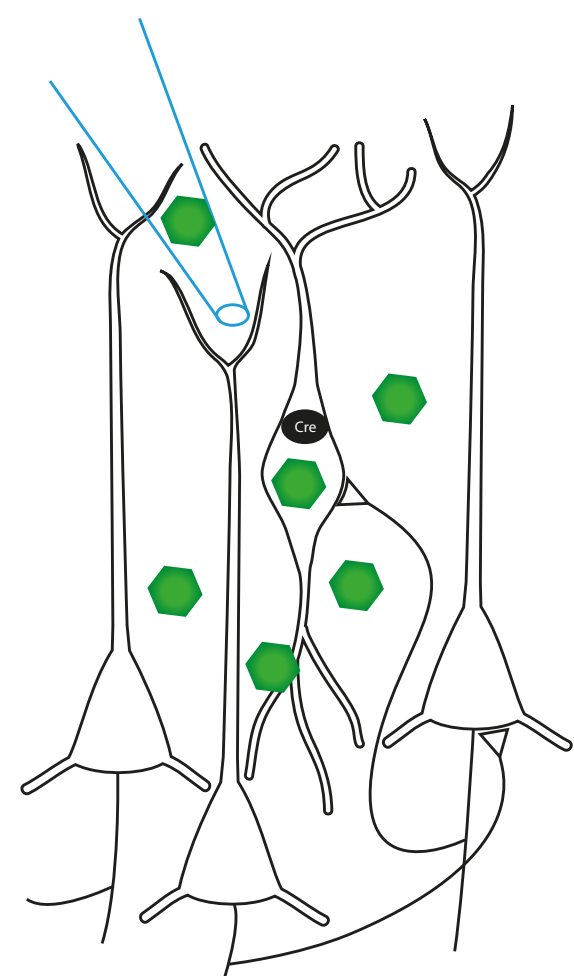

AAV8-FLEX-TVA ${ }^{66 T}$-EGFP-oG

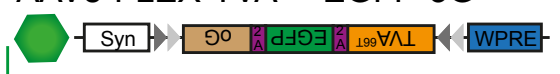

Day 1: Helper virus

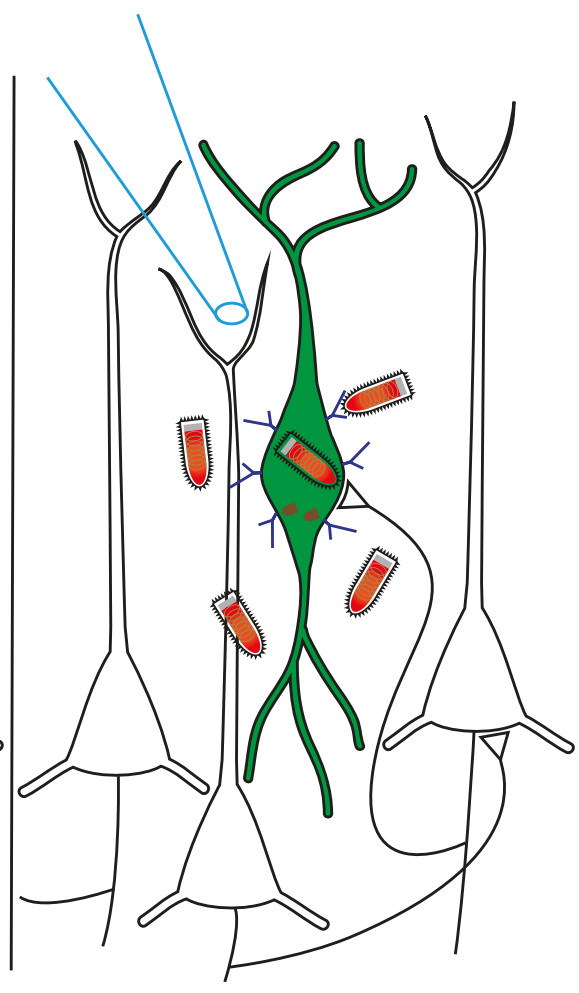

RV-SAD- $\triangle$ G-mCherry (EnvA)

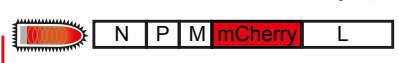

Day 15: Rabies virus

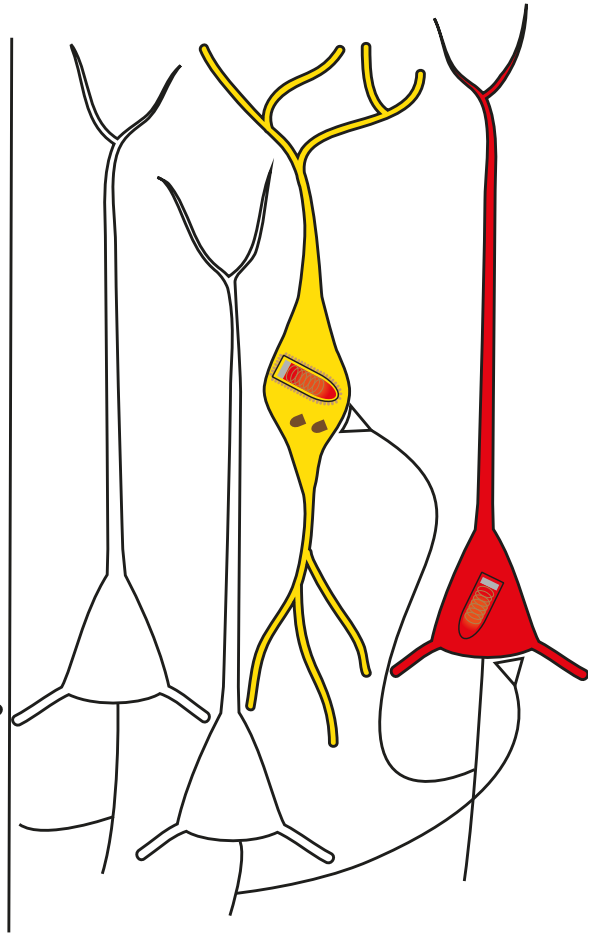

- optimized rabies glycoprotein TVA $^{66 \mathrm{~T}}$

$Y$ (mutated EnvA-R)

Figure 15: Experimental workflow of Cre-dependent RV-tracing of VIP neurons

On day 1, AAV8-FLEX-TVA ${ }^{66 T}$-EGFP-oG is injected into the barrel cortex of a VIP-Cre mouse. TVA66T is a mutated version of TVA, to which EnvA has a reduced affinity. RV-mCherry is injected 14 days later at the same location. This RV is pseudotyped with the TVA-ligand EnvA. Only cells expressing a high amount of TVA66T will be transduced by RV-mCherry. This will only be the case for Cre-expressing VIP cells. Because VIP cells co-express oG, RV-mCherry can incorporate the glycoprotein into its envelope and spread to first-order presynaptic neurons but not any further. While starter VIP cells appear yellow due to the mixture of mCherry and EGFP, presynaptic neurons appear exclusively red.

labeling at the injection site as well as in the VPM. The VPM reliably sends projections to the barrel cortex. Labeling in the VPM would indicate transsynaptic spread of RV.

Upon injection of AAV1-FLEX-TVA-EGFP-G and RV-mCherry we observed moderate amounts of RV-mCherry positive cells at the injection site and sparse labeling in the VPM (Figure 16C) The labeling in the VPM can only be explained by a Cre-independent leak expression of both TVA and G protein, so that RV could enter and spread from a cortical cell that receives thalamic input. The labeling at the injection site can be explained both by leak of TVA and $G$, as well as just TVA. If both proteins are leaky, the RV-mCherry positive cells are transsynaptically infected cells. However, leak expression of TVA alone could promote direct uptake of RV (Mijamichi et al., 2013). Therefore, at the injection site RV-mCherry positive cells are either directly or transsynaptically labeled. Because leak expression of both TVA and $G$ would confound local and long-range input tracing in Cre-expressing animals, this AAV construct was considered not useful to trace cell-type specific inputs.

Injection of AAV1-FLEX-TVA-mCherry, AAV8-FLEX-oG together with RV-EGFP resulted in sparse 
RV-EGFP labeling at the injection site but no labeling in the VPM (Figure 16D). Therefore, the AAVs do not express oG in the absence of Cre but TVA, so that RV can enter TVA positive cells directly at the injection site. Therefore, these constructs would produce error prone results when analyzing local connectivity but not long-range connectivity.

The combination of AAV1-FLEX-tTA-EGFP-TVA, AAV1-TREtight-BFP-G and RV-mCherry as well as the combination of AAV1/2-FLEX-mCherry, AAV1/2-FLEX-G-TVA and RV-EGFP resulted in strong RV labeling at both the injection site and in the VPM (Figure 16E, F). Therefore, these constructs show a strong leak expression of both TVA and G. They are not suited for cell-type specific tracing.

Considering that the leak of TVA seems to be much stronger and that the leak of $G$ can only take effect if TVA leak occurs, we thought of using a construct, with which the leak of TVA can be reduced.

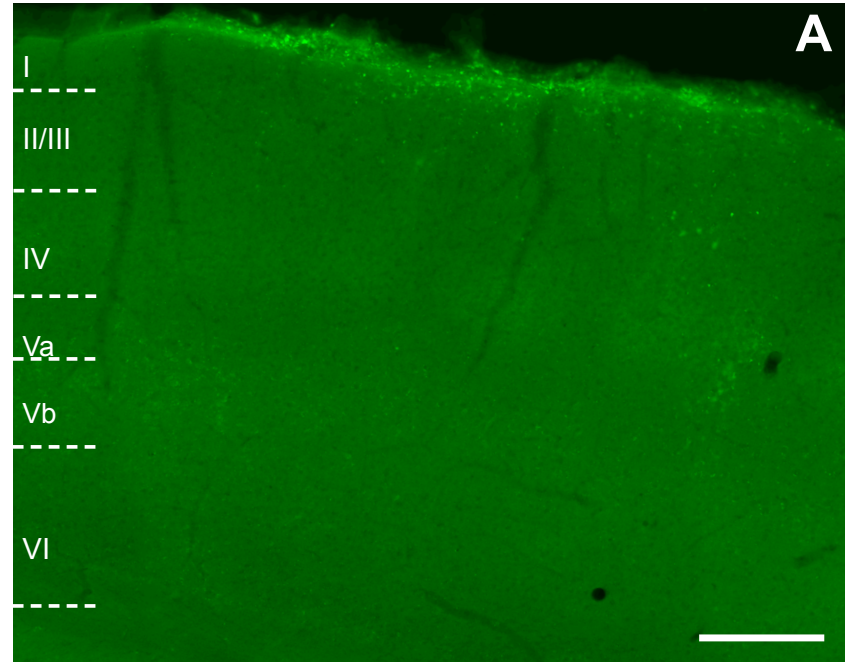

RV-SAD- $\triangle$ G-EGFP (EnvA)

\begin{tabular}{|l|l|l|l|l|}
\hline $\mathrm{N}$ & $\mathrm{P}$ & $\mathrm{M}$ & $\mathrm{EGFP}$ & $\mathrm{L}$ \\
\hline
\end{tabular}

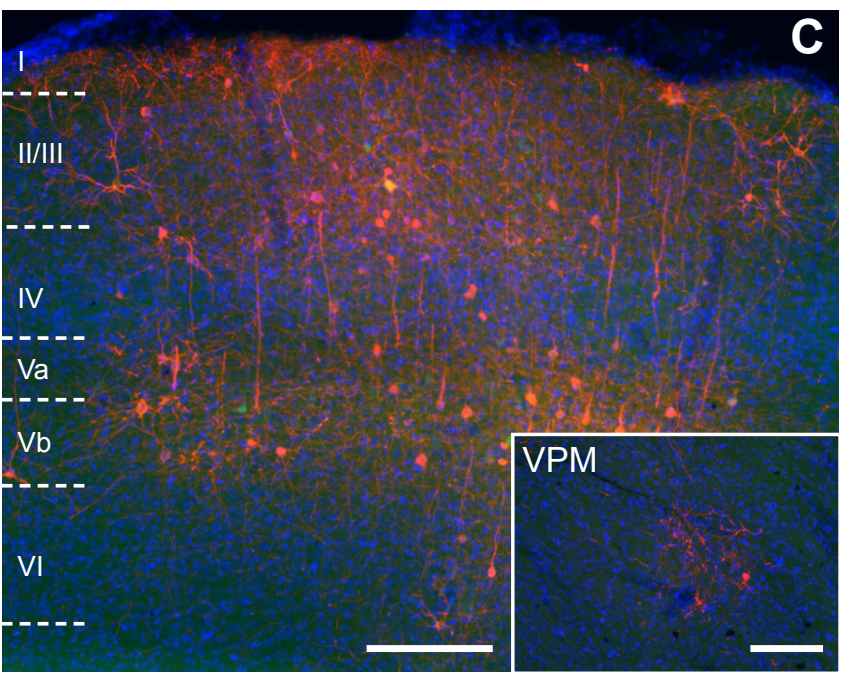

AAV1-FLEX-TVA-EGFP-G

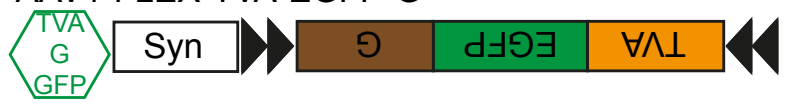

+ RV-mCherry

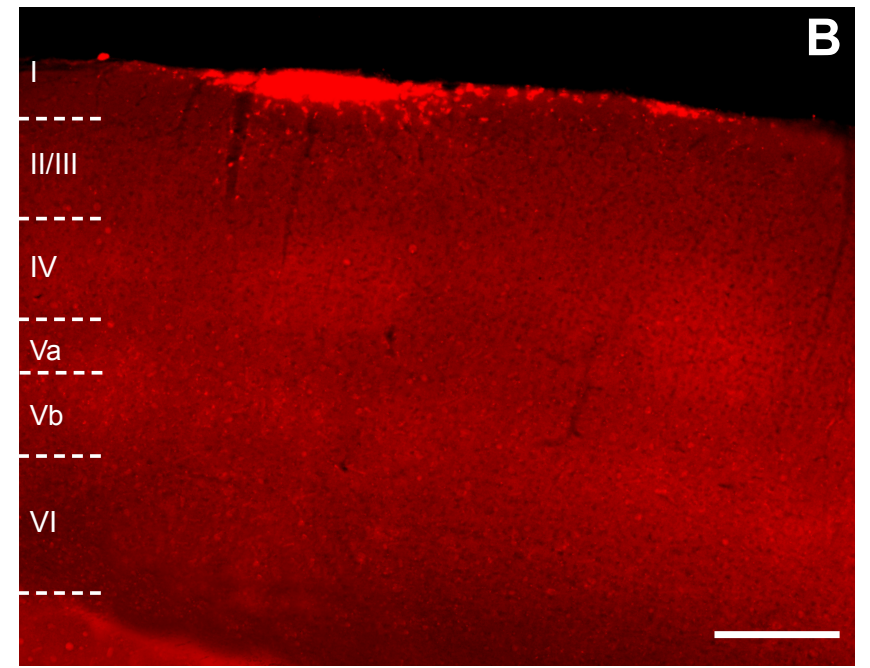

RV-SAD- $\triangle$ G-mCherry (EnvA)
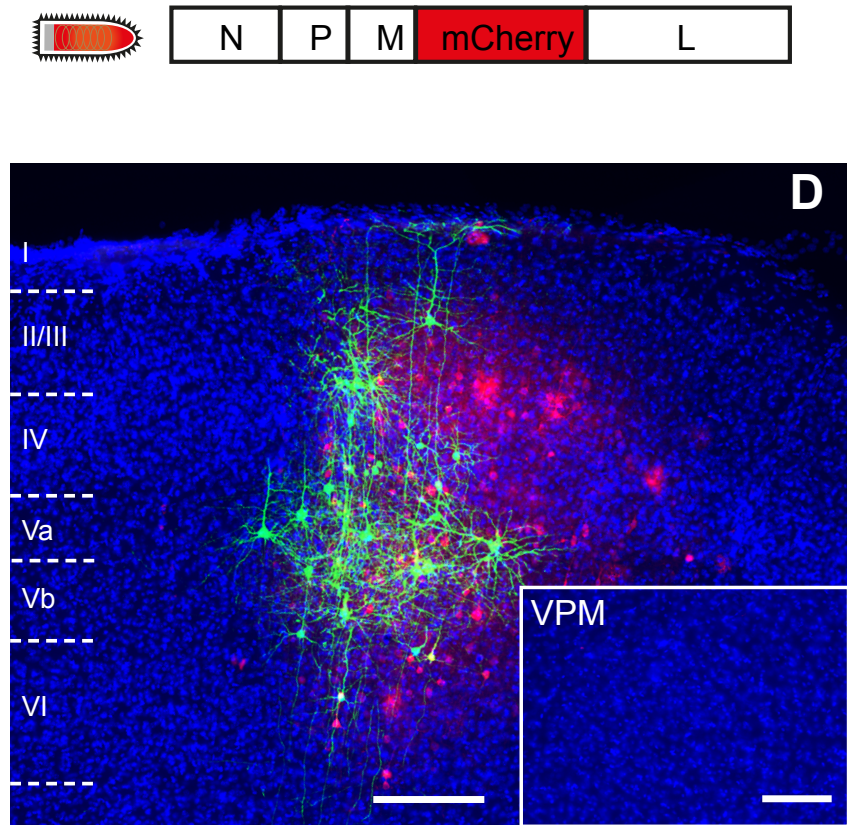

AAV1-FLEX-TVA-mCherry

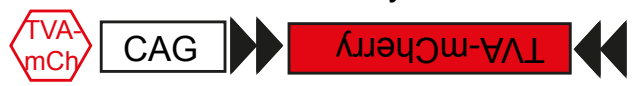

AAV8-FLEX-oG

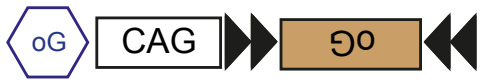

+ RV-EGFP 


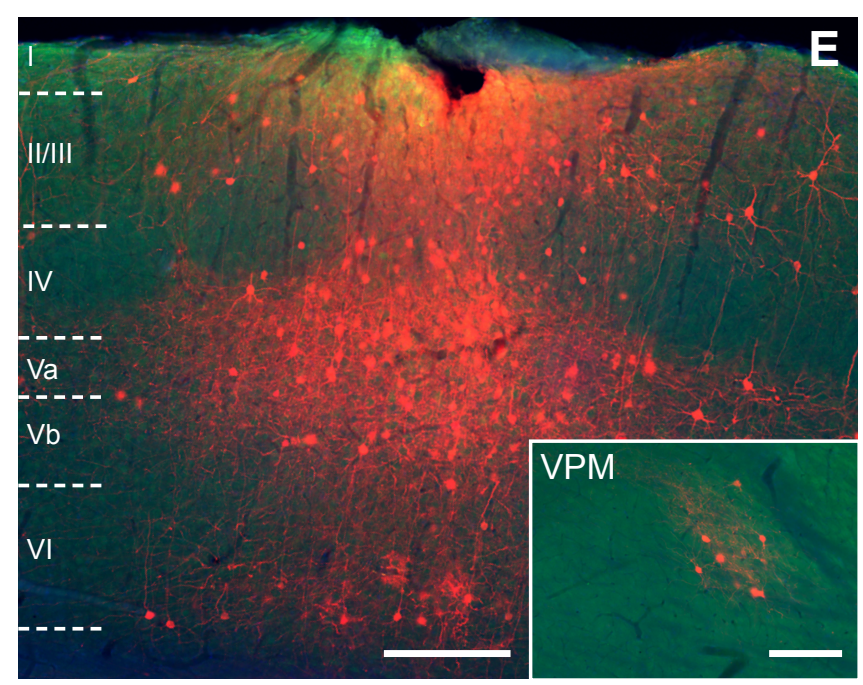

AAV1-FLEX-tTA-EGFP-TVA

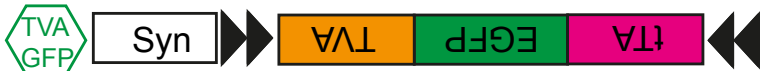

AAV1-TREtight-BFP-G

\begin{tabular}{|c|c|c|c|}
\hline $\begin{array}{c}\text { G } \\
\text { BFP }\end{array}$ & TRE & BFP & G \\
\hline
\end{tabular}

$+\mathrm{RV}$-mCherry

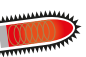

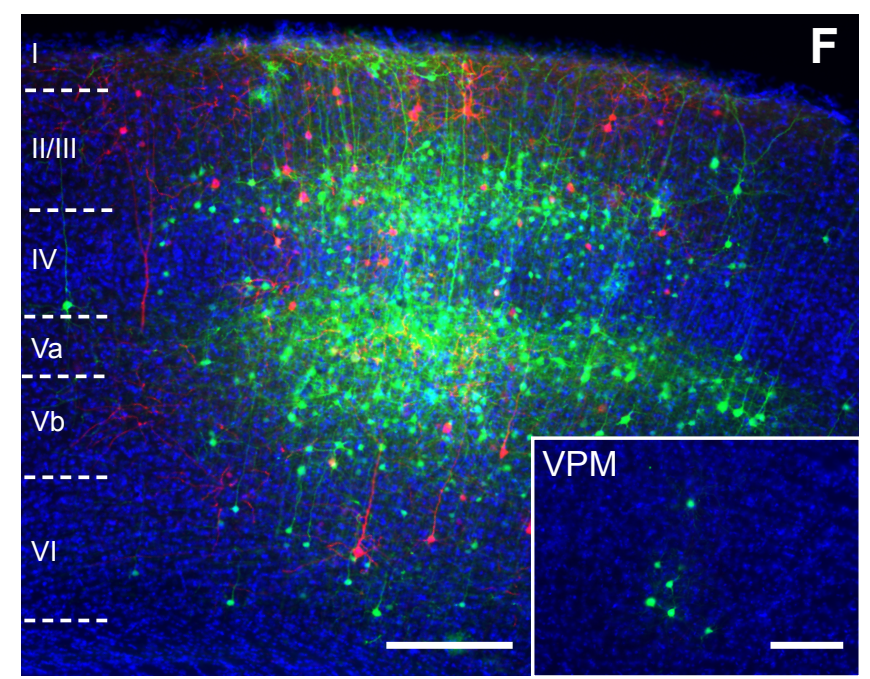

AAV1/2-FLEX-mCherry

mCh EF-1a Кщәчวш

AAV1/2-FLEX-G-TVA

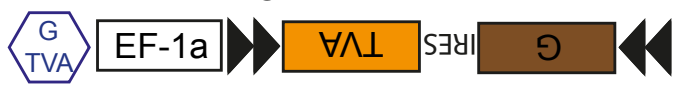

+ RV-EGFP

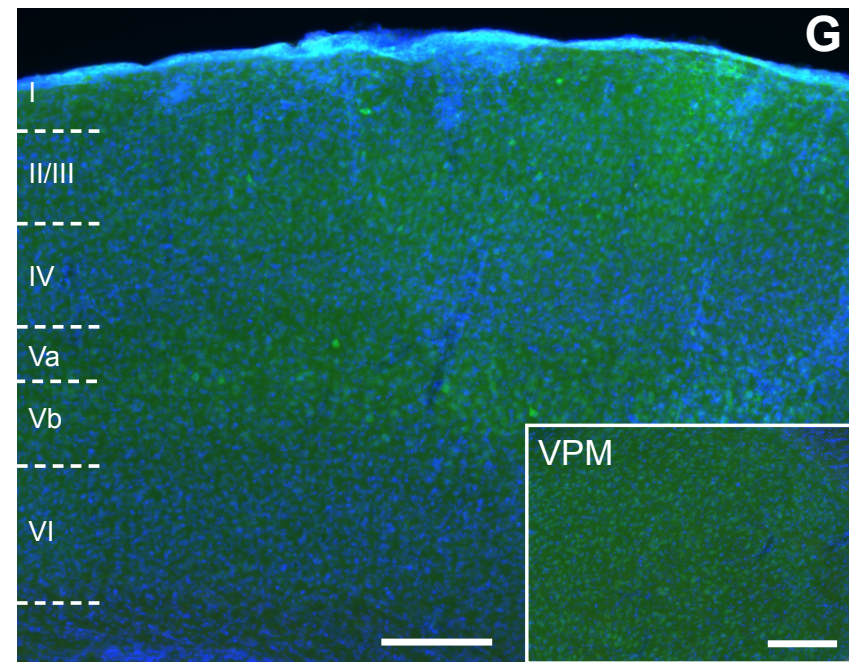

AAV8-FLEX-TVA ${ }^{66 T}$-EGFP-oG

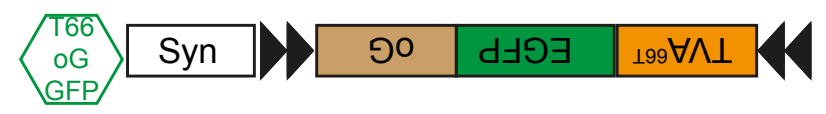

+ RV-mCherry

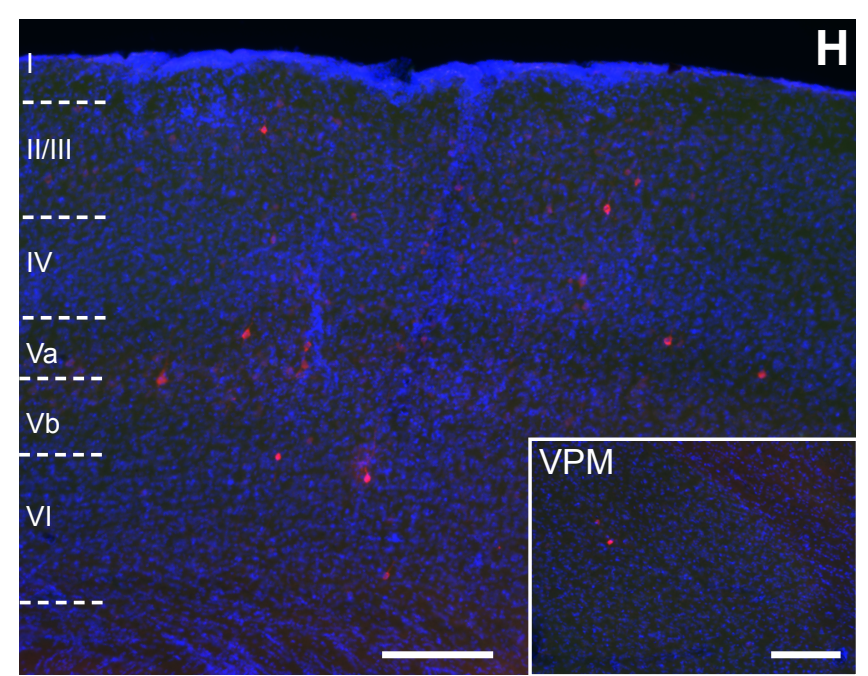

AAV1-FLEX-TVA ${ }^{66 T}$

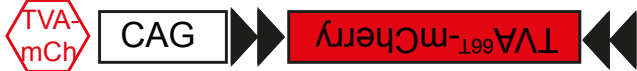

AAV8-FLEX-oG

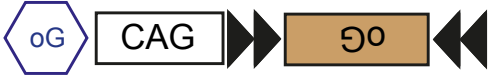

+ RV-EGFP

\section{Figure 16: Validation of Cre-dependent constructs for RV-tracing in BL6 animals}

(A, B) Coronal section through the barrel cortex of a BL6 mouse after injection of RV-EGFP or RV-mCherry, without prior injection of helper AAVs. In both cases, no transduced cells were detected. Uptake of RV into cells strictly depends on the presence of TVA (scale bar: $200 \mu \mathrm{m}$ ). (RVs were kindly provided by Karl-Klaus Conzelmann).

(C-F) We injected various Cre-dependent AAV helper virus constructs into barrel cortex of BL6 animals followed by RV. Although no Cre was expressed in these animals, we saw cellular labeling of helper and/or RV 
at the injection site. This can be mostly attributed to a leak of TVA, which allows for a direct uptake of RV. Labeling outside of the injection site is a sign of leak of rabies glycoprotein and indicative of transsynaptic spread. We looked for labeling in the ventral posteromedial nucleus of the thalamus (VPM), which provides very reliable input to the barrel cortex. We found weak to strong VPM labeling in three out of four tested constructs (scale bar: $200 \mu \mathrm{m}$ overview, $100 \mu \mathrm{m}$ VPM insert). (Viral constructs were kindly provided by lan Wickersham (C, E), Karl Deisseroth (D) and Martin Schwarz (F)).

$(\mathbf{G}, \mathbf{H})$ When using vectors with the mutated version TVA ${ }^{66 T}$ instead of the conventional TVA, no Cre-independent labeling of RV was visible. TVA ${ }^{66 T}$-mCherry packaged into AAV1 showed occasional retrograde transport apparent due to labeling in the VPM. We decided to use for all subsequent experiments TVA ${ }^{66 T-E G F P-o G ~}$ packed into AAV8 because it tags both TVA ${ }^{66 T}$ and $\mathrm{OG}$, and did not show any retrograde transport. (Viral constructs were kindly provided by Edward Callaway)

TVA has a very high sensitivity towards EnvA so that a minimal leak expression of TVA is already sufficient for uptake of RV into the cell (Mijamichi et al., 2013; Rong et al., 1998). Mijamichi et al. (2013) engineered a mutated version of TVA, in which they replaced glutamate at the 66th position of the amino acid chain with threonine. The resulting mutated TVA ${ }^{66 T}$ has a much lower affinity to EnvA and thus cells need to express a higher amount of TVA ${ }^{66 T}$ to allow for RV uptake.

We tested two different viral constructs with TVA ${ }^{66 T}$ in BL6 animals (Figure 16G, H). AAV8-FLEXTVA $^{66 T}$-EGFP-oG and RV-mCherry injection yielded a few weakly EGFP-positive cells at the injection site but no RV positive cells at neither the injection site nor the VPM. Similarly, injection of AAV1-FLEXTVA $^{66 T}$-mCherry, AAV8-FLEX-oG and RV-EGFP resulted in a few mCherry labeled cells but no RV transduction was apparent. However, there were a few mCherry positive cells in the VPM that most likely resulted from a retrograde transport of AAV1-FLEX-TVA-mCherry along thalamocortical fibers. These results clearly demonstrate that the mutated TVA ${ }^{66 \mathrm{~T}}$ abolishes all leak expression and promises to restrict $\mathrm{RV}$ tracing exclusively to Cre-expressing cells.

We decided to use the AAV8-FLEX-TVA ${ }^{66 T}$-EGFP-oG (Figure 16G) for our experiments in VIP-Cre mice for two reasons. First, it allows to unequivocally identify starter cells because TVA and $G$ are in the same vector. Second, it does not show any retrograde transport which otherwise might result in the generation of starter cells outside of the injection site. All subsequent experiments described were carried out with the same batch of AAV8-FLEX-TVA ${ }^{66 T}$-EGFP-oG and RV-mCherry tested in controls.

\subsubsection{Starter cells in reeler do not show a laminar bias}

VIP-Cre and VIP-Cre/reeler mice ( $\mathrm{n}=6$ in each group) were injected with AAV-TVA ${ }^{66 T}$-EGFP-oG and two weeks later with RV-mCherry into the right barrel cortex. The virus injections were centred on the $\mathrm{C} 2$ barrel related column as in previous experiments. The C2 column was localized with ISOI in WT and reeler animals. Despite the absence of layers, the topology of whiskers is preserved in reeler and whisker stimulation in reeler elicits highly localized hemodynamic responses that are comparable in temporal dynamics, size and strength to WT animals (Guy et al., 2015). Therefore, we could target our viral injections to the same functional module of the barrel cortex in WT and in reeler mice to have optimal comparability between genotypes. 

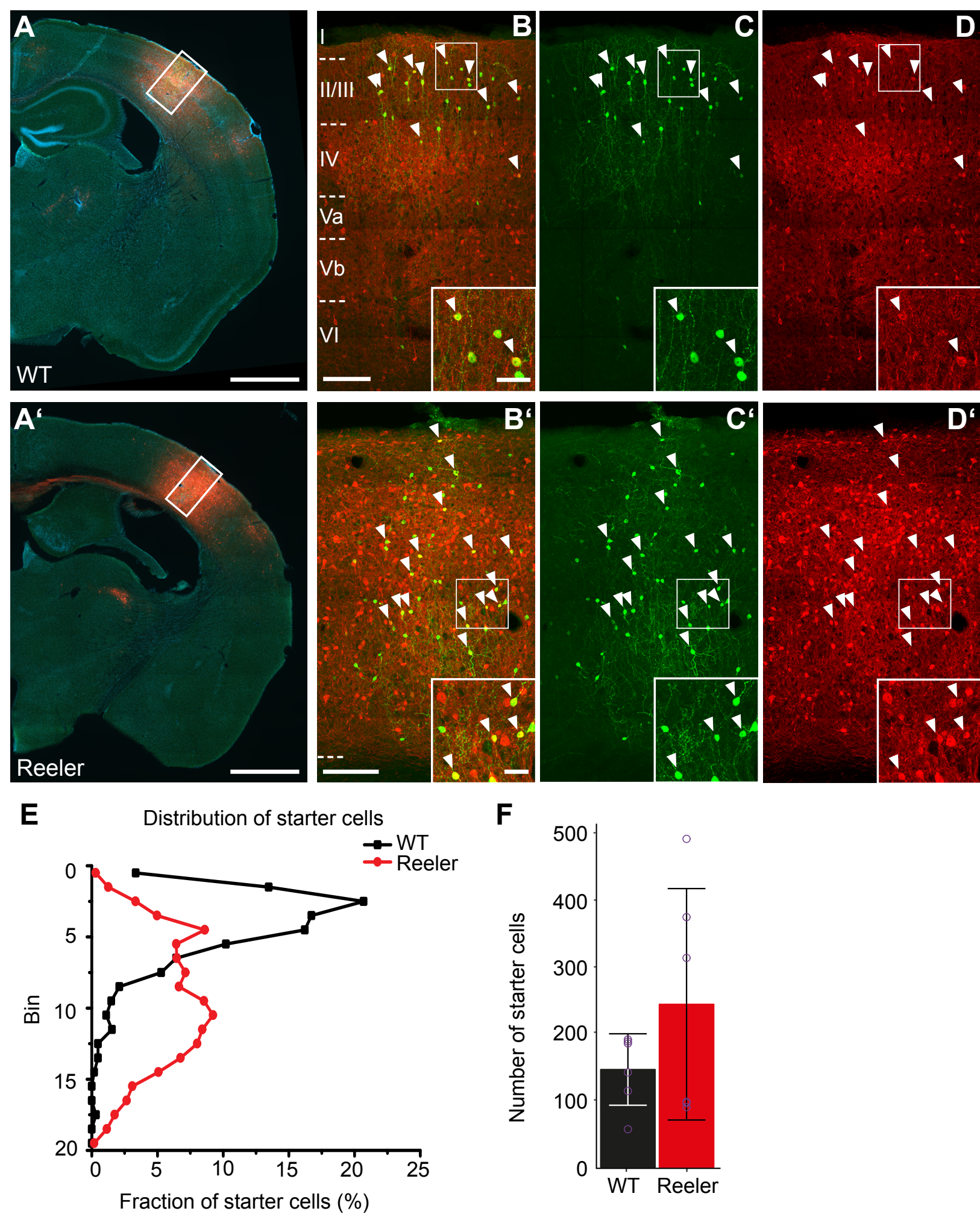

Figure 17: Identification of starter cells in VIP-Cre and VIP-Cre/reeler mice

$\left(\mathbf{A}, \mathbf{A}^{\prime}\right)$ Coronal section through an injection site in right barrel cortex of WT and reeler mice (scale bar: $1000 \mu \mathrm{m})$.

(B, B') Insert in A, A'. Images show maximum projections of confocal stacks. Cells marked by white arrowheads are double-labeled starter cells that have been co-transduced by AAV-TVA66T-EGFP-oG and RV-mCherry. Inserts in B, B' show some of these cells in higher resolution (scale bar overview: 100 $\mu \mathrm{m}$; scale bar insert: $20 \mu \mathrm{m})$.

(C, C') VIP neurons transduced by AAV-TVA66T-EGFP-oG. In WT they show the typical bipolar mor- 
phology of VIP neurons and are most abundant in LII/III. In reeler they have a more multipolar morphology and do not show a clear layer bias.

(D, D') Exclusively RV-mCherry positive cells are local presynaptic cells to the starter cells. These local inputs are very numerous and form a dense network of cell bodies and neuropil. Because of their sheer abundance we did not analyze the local inputs.

(E) Distribution of starter cells across the cortical depth for WT and reeler. Cortical thickness was divided into 20 equal-sized bins. The proportion of starter cells in each bin was plotted. While in WT starter cells were predominantly in the upper third, starter cells in reeler were more dispersed ( $n=6$ per group).

(F) Number of starter cells in each genotype. Purple circles represent individual animals. Number of starter cells are not significantly different ( $n=6$ per group; Mann-Whitney rank sum test, $U=15, p=0.70$; mean \pm SD)

One week after RV-mCherry injection, animals were sacrificed and brains sectioned coronally (Figure 17A, $A^{\prime}$ ). Cells double labelled with EGFP and mCherry were considered starter cells and counted on each section spanning the injection site. As expected from the distribution of VIP cells in reeler we observed no bias towards upper layers of starter cells in reeler mice (Figure 17B'-D'), unlike in WT mice (Figure 17B-D). To visualize the distribution of starter cells in the two genotypes, we divided the cortex in 20 equal bins, each one about $50 \mu \mathrm{m}$ thick and calculated the proportion of starter cells in each bin relative to the total number of starter cells (Figure 17E). While in WT there was a clear bias of starter cells towards the upper third of the cortex, the distribution of starter cells in reeler was broader with the majority of cells in the middle part of the cortex.

When counting the total number of starter cells, we observed a bias with reeler brains having on average more starter cells but the difference was not significant (Figure 17F; mean WT: $146.2 \pm 53.4$; mean Reeler: $234.7 \pm 173.0$; Mann-Whitney rank sum test, $U=15, p=0.70$ ). This difference could arise because brain tissue in reeler might absorb virus solution better due to its lower integrity. The potential confounder of such a bias is addressed below.

\subsubsection{VIP cells receive qualitatively similar long-range input in WT and reeler}

To address the question if the absence of layers impacts the capacity of VIP cells to sample long-range inputs, we analyzed the transsynaptically labeled cells in the entire brain. We manually counted all RV-labeled cells on all sections from Bregma +3 to $-4.5 \mathrm{~mm}$ but omitted cells in the barrel cortex itself because of their extreme abundance. The Allen Brain atlas was used as a reference. Each coronal section was overlaid with the corresponding atlas section. RV-mCherry positive cells were assigned to an area based on the outline of the atlas and the cytoarchitectonic features discernable with nuclear stain. It is important to mention that areal boundaries are preserved in reeler despite the mislamination (Boyle et al., 2011). Thus, the Allen Brain atlas provides a legitimate reference for the reeler brain architecture, too.

Examples for areas in which we found transynaptically labelled cells are presented in Figure 18. 


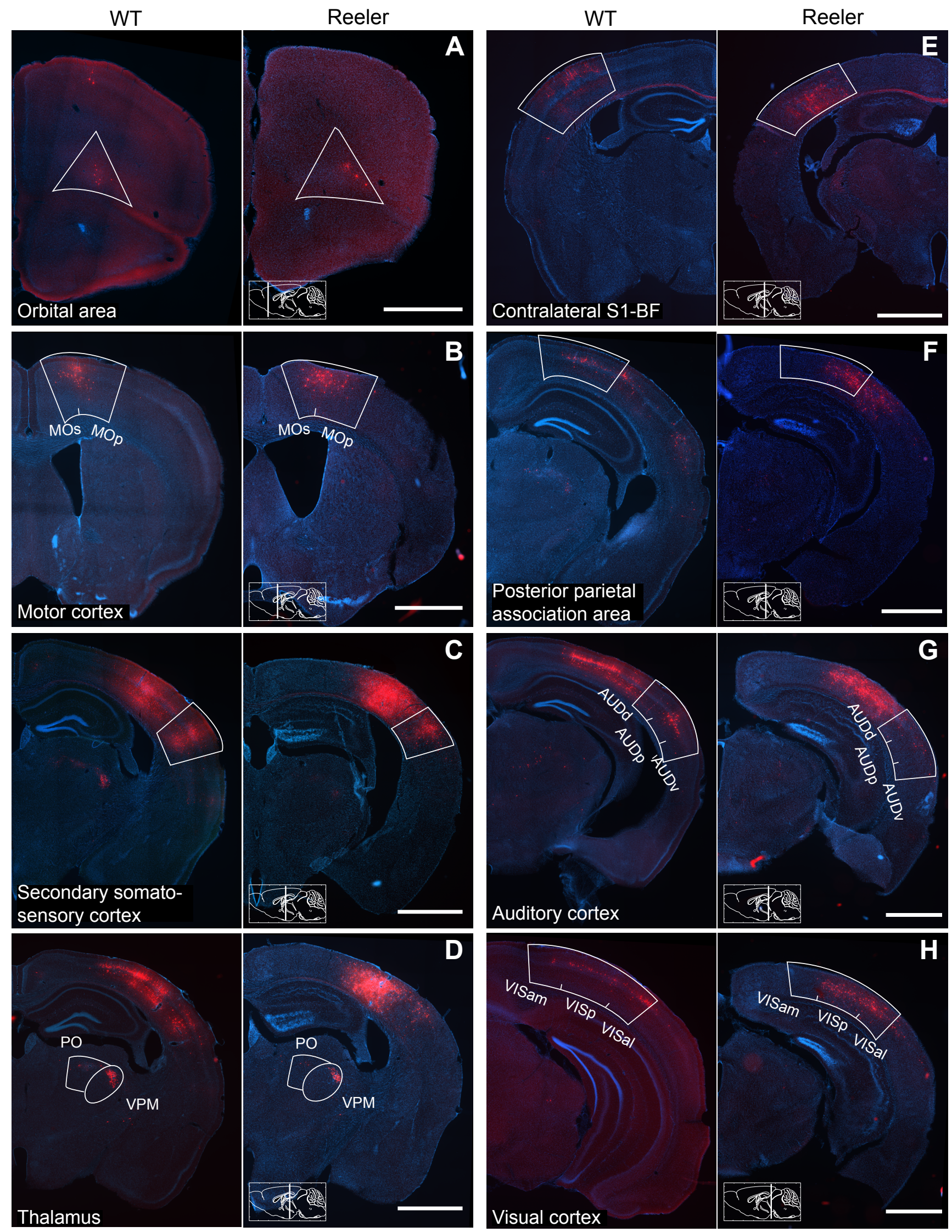




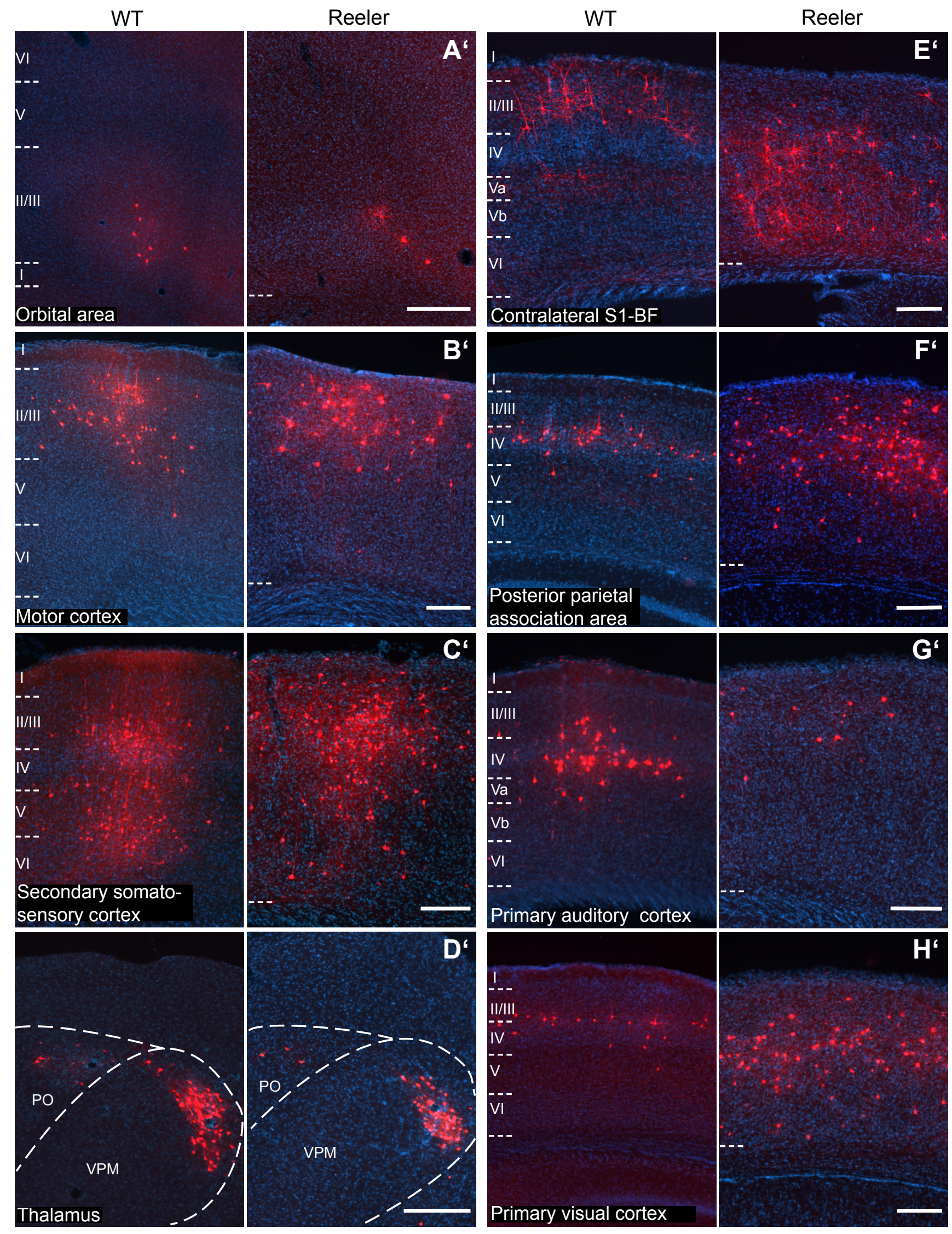


Figure 18: Long-range input to VIP cells in barrel cortex of WT and reeler mice

(A-H) Coronal sections along the rostro-caudal extent of WT (left) and reeler (right) mice. Images show consistently labeled areas with presynaptic partners of VIP cells. The white contour delineates the boarders of the area written in the left bottom corner. Section planes are indicated on the schematic sagittal brain section in the bottom middle (scale bars: $1000 \mu \mathrm{m}$ ).

(A'-H') Close-ups of the overview imaged in A-H. Contralateral barrel cortex contained more cells in reeler that are located in deeper parts of the cortex compared to WT (E'). Primary auditory cortex contained a smaller number of cells in reeler (G'). All other areas showed a comparable number of cells and even a fairly similar distribution. Projection neurons in reeler appeared to have slightly larger somata (scale bars: 200 $\mu \mathrm{m})$.

(Abbr.: AUDd, dorsal auditory area; AUDp, primary auditory area; AUDv, ventral auditory area, MOp, primary motor cortex, MOs, secondary motor cortex; PO, posterior complex of the thalamus; S1-BF, primary somatosensory cortex barrel field; VISal, anterolateral visual area; VISam, anteromedial visual area; VISp, primary visual area; VPM, ventral posteromedial nucleus of the thalamus).

We did not detect inputs from areas that were exclusive to one genotype. Therefore, VIP neurons in reeler mice receive input from the very same areas as WT mice However, we noticed that the number of inputs from the contralateral hemisphere was consistently higher in reeler (Figure 18E, E') and the number of inputs from the auditory cortex was consistently lower (Figure 18G, G'). Therefore, our data suggest that the absence of layers could affect the magnitude and the proportion of long-range inputs to VIP cells originating from specific cortical sources.

\subsubsection{VIP cells in reeler receive less input per cell}

Next we analyzed if there is a quantitative difference in the inputs between genotypes. First we focused on global inputs (Figure 19A, Table 1). We summed up all long-range inputs from ipsilateral and contralateral cortical areas, from the thalamus and from all subcortical areas. We normalized the inputs by dividing the cell count with the number of starter cells to calculate the input magnitude. This is taken as a proxy for the strength of an input. VIP neurons in reeler received less overall inputs per cell. Similarly, they received less cortical inputs and less ipsilateral cortical inputs. However, they received more contralateral inputs per cell. There was no difference for global subcortical or thalamic input.

The input magnitude might be influenced by the number of starter cells. One presynaptic neuron could contact multiple starter cells. This divergent connectivity prompts that with a rising number of starter cells, the count of additionally labeled postsynaptic cells will drop. Hence, the higher the number of starter cells, the lower will be the ratio of starter cells to input cells (input magnitude). The smaller overall input magnitude in reeler might be because this group has more starter cells on average. To investigate this relationship we plotted the number of starter cells against the input magnitude for the two genotypes (Figure 19B). A negative slope of the regression line would indicate a negative relationship between starter cells and input magnitude. The slope was not different between genotypes and close to zero in both cases (WT: Input magnitude $=-0.015^{*}$ starter cells $+35.89, R^{2}=0.006, p=.89$; Reeler: Input magnitude $=-0.00085^{*}$ starter cells $+20.42, R^{2}=0.0004, p=0.97$ ). In consequence, there is no indication 

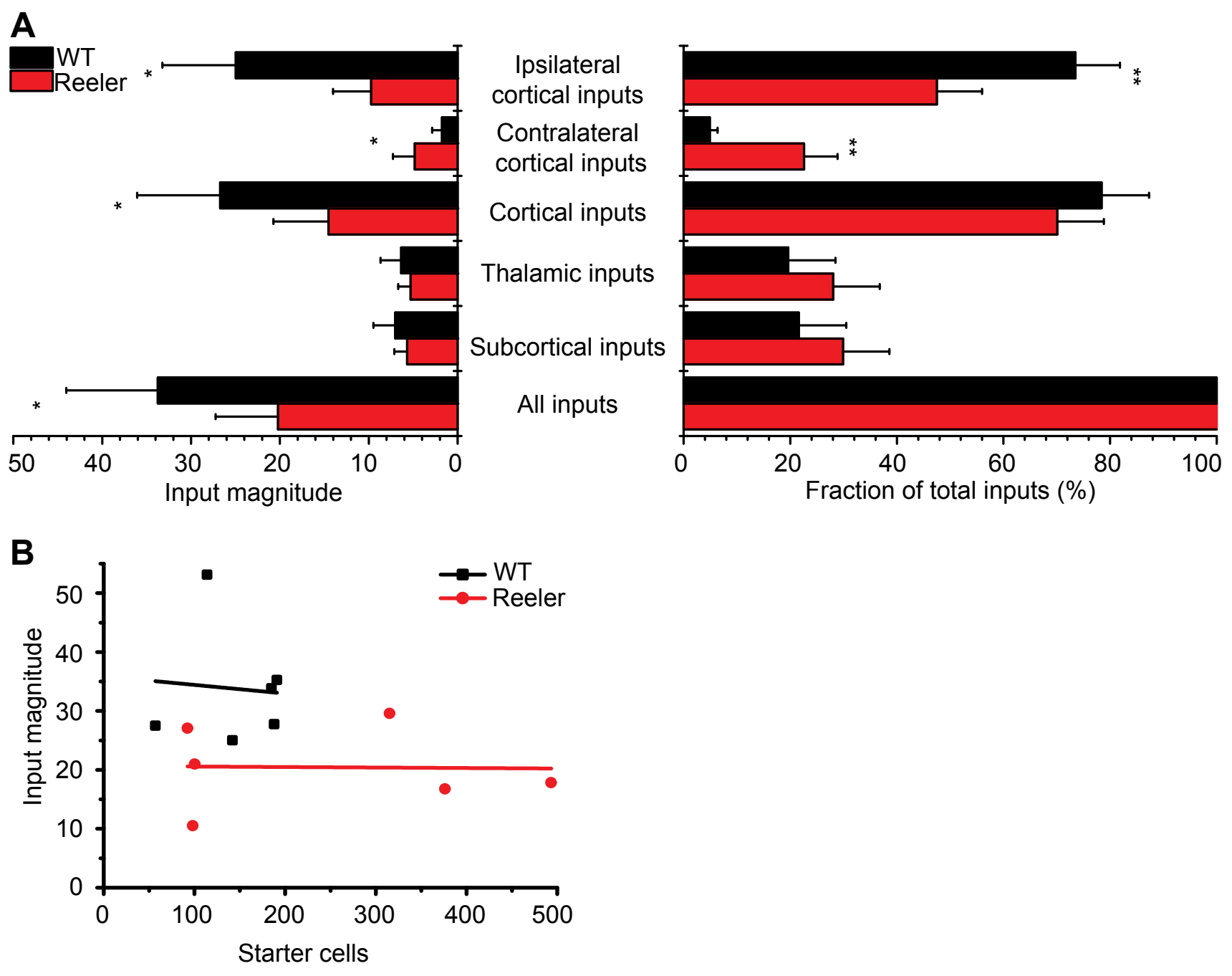

Figure 19: Normalized inputs from global cortical and subcortical areas

(A) Histograms representing the input magnitude (input cell number/starter cell number) and fraction of total inputs from summed up cell counts of global brain areas (mean $\pm S D$ ). $* p<0.05, * * p<0.01$

(B) Plot of input magnitude against starter cell count for two genotypes. There is no correlation between the two variables. This indicates that differences in the input magnitude are not influenced by the number of starter cells.

that the divergence of presynaptic neurons is so prominent that it distorts the input magnitude. The higher number of starter cells in reeler cannot explain the lower input magnitude. Instead, the evidence points to a lower number of presynaptic neurons converging onto VIP neurons in reeler.

Because the overall input magnitude was different between the genotypes, the subsequent analyzes for differences among individual areas using the input magnitude would be inherently biased. Therefore, we employed another means of normalization, too, which is independent of the number of inputs: input fraction. It is calculated by dividing the number of inputs in an area by the number of total inputs in the brain. It is a measurement for how different inputs are balanced. Looking at the global inputs from ipsi- and contralateral cortex as well as from subcortical areas we noticed a significantly higher fraction of contralateral inputs and a significantly lower fraction of ipsilateral inputs in reeler (Figure 19A). Interestingly, the inputs from the hemispheres added up in a way that total cortical input fraction was not different between genotypes. Looking at the fraction of subcortical input, reeler mice received a slightly but non-significantly higher fraction of global subcortical as well as thalamic input. 


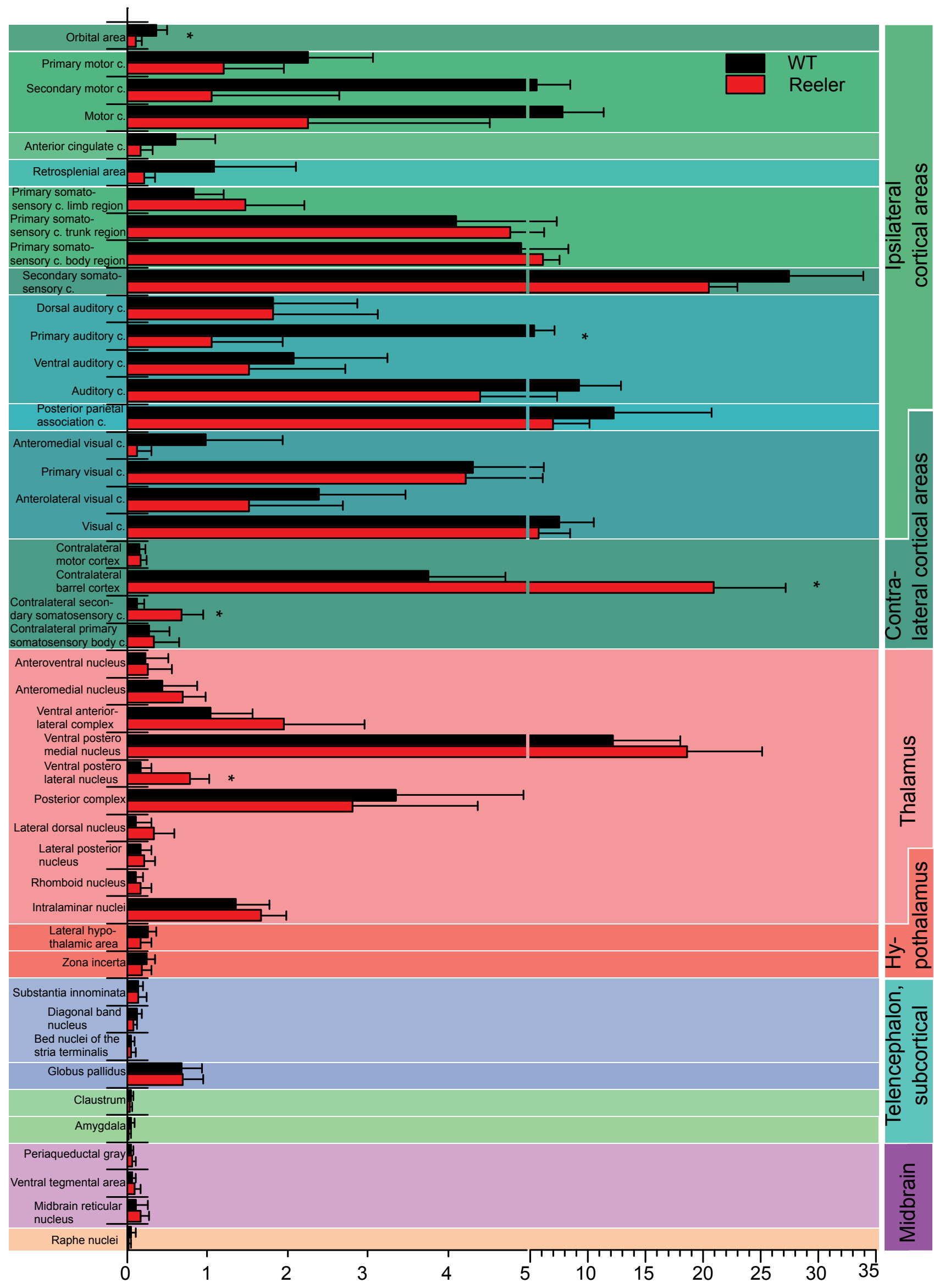

Fraction of total inputs (\%) 
Figure 20: Comparative analysis of the fraction of inputs from individual areas

Mean proportion of RV-labeled cells in 41 individual areas normalized against the total number of inputs in the whole brain for the two genotypes. For motor cortex, primary somatosensory cortex body region, auditory cortex and visual cortex the summated proportions of the individual subareas are shown as well. Pairwise comparisons were carried out to assess differences in input fraction for individual areas. For individual values see Table 2 (mean \pm SD; Abbr.: c, cortex). ${ }^{*} p<0.05$

In sum, it seems that VIP neurons in reeler compensate for the smaller proportion of ipsilateral cortical inputs with a higher proportion of contralateral inputs.

\subsubsection{VIP neurons in reeler mice receive more homotopic contralateral input}

We analyzed if the input fraction of individual areas is different between genotypes. We selected 41 consistently labeled areas that constituted the majority of inputs (97.3\% in WT; $98.6 \%$ in Reeler). We calculated the input fraction for each area and made pairwise comparisons between genotypes. Data showing mean, standard deviation and p-values for each area and genotype are listed in Table 2 and visualized in Figure 20. For motor cortex, somatosensory cortex body region, auditory cortex and visual cortex we also summed up counts in the individual primary and secondary areas to calculate a combined input fraction for better visualization. Orbital area and the primary auditory cortex accounted for a smaller proportion of inputs in reeler. The contralateral barrel cortex as well as the contralateral secondary somatosensory cortex and the ipsilateral ventral posterolateral nucleus of the thalamus contributed a higher proportion of inputs in reeler.

\subsubsection{Only contralateral projection neurons in reeler show a different lami- nar distribution}

In reeler mice, the whole cortex shows alterations because of reelin deficiency. This means that not just the VIP starter cells are malpositioned but all their cortical presynaptic cells, too. However, the pattern of cellular malpositioning varies along the rostro-caudal axis (Boyle et al., 2011; own unpublished observation). In motor cortex cells are disorganized with elements of inversion of deep layer cells and normal migration of upper layer cells (Boyle et al., 2011; Dekimoto et al., 2010). In S1 cells are completely disorganized (Wagener et al., 2010). In visual cortex cells acquire a "mirror-image type of architecture" centered around LII/III cells (Boyle et al., 2011).

We wanted to investigate if the projection neurons labeled in our study in the different areas are different from WT and follow the known patterns of cellular dispersion in reeler. We divided the cortex into 20 equally sized bins and counted the proportion of presynaptic cells in each bin for ipsilateral primary motor cortex, primary sensory areas, secondary somatosensory cortex and contralateral barrel cortex (Figure 21). All ipsilateral areas had a surprisingly similar distribution of projection neurons between WT and reeler. In visual cortex of WT brains, most projection neurons were located around 

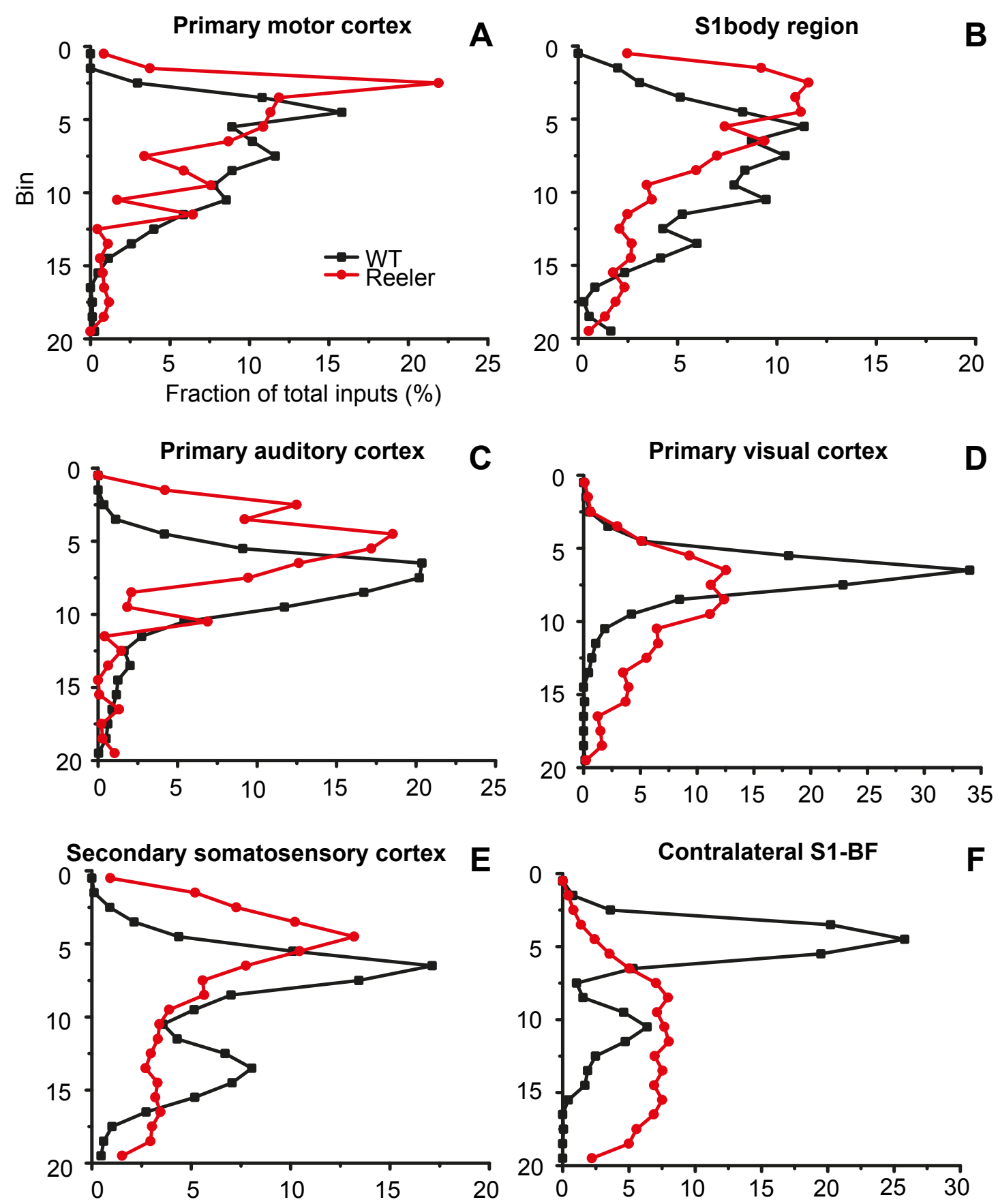

Figure 21: Distribution of projection neurons in cortical input areas

(A-F) The thickness of the cortex was divided into 20 equal sized bins. The fraction of inputs in a bin was normalized against the total inputs from this cortical area and was plotted. While ipsilateral areas showed a similar distribution of projection neurons between the two genotypes, the distribution of contralateral projection neurons from the barrel cortex was visibly different. In WT, projection neurons were predominantly in the upper third of the cortex, while in reeler they were predominantly in the lower two thirds of the cortex. (Abbr.: S1, primary somatosensory cortex; BF barrel field)

the LIII-LIV border. This peak was smoothed out in reeler, indicating that the projection neurons were rather dispersed than inverted in their arrangement (Figure 21A-E). The only pattern that was visibly different between genotypes was in contralateral barrel cortex (Figure 21F). In WT, projection neurons were predominantly located in the upper third of the cortex corresponding to LII/III. In reeler, projection neurons were predominantly located in the lower two thirds of the cortex. 
In conclusion, in reeler mice the inputs to VIP cells from ipsilateral cortical areas are less numerous and arranged similarly as in WT. On the contrary, in reeler mice inputs from contralateral barrel cortex to VIP cells are more numerous and differently arranged compared to WT. 


\section{DISCUSSION}

Unraveling the organization of brain-wide circuits is an important step in understanding how different brain areas interact to generate unified perception and directed action. Many studies have investigated the brain-wide inputs to the primary somatosensory cortex in mice using classical tracers (Aronoff et al., 2010; Baskerville et al., 1997; Fabri and Burton, 1991; Larsen et al., 2007; Lee et al., 2008; Mao et al., 2011; Miller and Vogt, 1984; White and DeAmicis, 1977). Their results highlight the vast interconnectivity of the barrel cortex with other cortical and subcortical areas. However, the virtue of specificity with which these long-range connections innervate different types of neurons has been an unexplored dimension. Therefore, it is essential to comparatively examine the patterns of input to different classes of cortical neurons. In this thesis we examined the brain-wide monosynaptic inputs to GABAergic PV neurons in mouse barrel cortex as well as the inputs to VIP neurons in barrel cortex of wildtype and reeler mutant mice. For both projects we developed new tools in a collaborative effort to minimize potential confounders of this tracing technique.

First, we developed intersectional rabies virus tracing, to map local and long-range inputs to GABAergic PV cells. The findings are graphically summarized in Figure 22. Local inputs from within the barrel cortex outweighed long-range inputs by about ten-fold. Local inputs were mainly excitatory, and LIV was the most prominent input layer. LI inhibitory cells connected to LII/III PV neurons. Long-range inputs originated mainly from other sensory cortices and the thalamus. Surprisingly, in visual cortex LIV contained the most projection neurons, questioning the role of LIV as a mere input layer. Although PV neurons received input from the same sources as other inhibitory neurons (Wall et al., 2016), the layer-specific circuit motifs hint that studies need to look at other levels than just the area of input to reveal the full complexity of connectivity.

Second, we optimized Cre-dependent RV tracing to make it more specific and efficient. With the optimized construct we comparatively mapped the long-range inputs to VIP neurons in barrel cortex of WT and reeler mice. The findings are graphically summarized in Figure 23. VIP neurons received input from the same areas in both genotypes, mostly from other sensory cortices, motor cortex, posterior parietal association area and the thalamus. However, while the magnitude of subcortical input was not altered, we found profound differences in the magnitude of cortical inputs. VIP neurons in reeler mice received a much lower number of inputs from other ipsilateral cortical areas and a much higher number of inputs from the contralateral hemisphere. Previous anatomical studies on the reeler mouse have never assessed quantitative differences in connectivity so that this striking shift in the balance of ipsivs. contralateral inputs has been overlooked and assigns the reeler mouse a quite distinct connectivity scheme.

Because these two projects are densely intertwined with the question if different classes of GABAergic neurons receive a different pattern of input, this topic will be addressed as well. We conclude based on this and many other studies that the main subtypes of GABAergic neurons all receive very similar input. Therefore, we emphasize the importance to improve tracing and analysis methods to detect more subtle differences and to assess the strength of connections with physiological techniques. 
AAV8 - Con/Fon - TVA-mCherry

AAV8 - Con/Fon - oG

RV-SAD- $\Delta$ G-EGFP (EnvA)

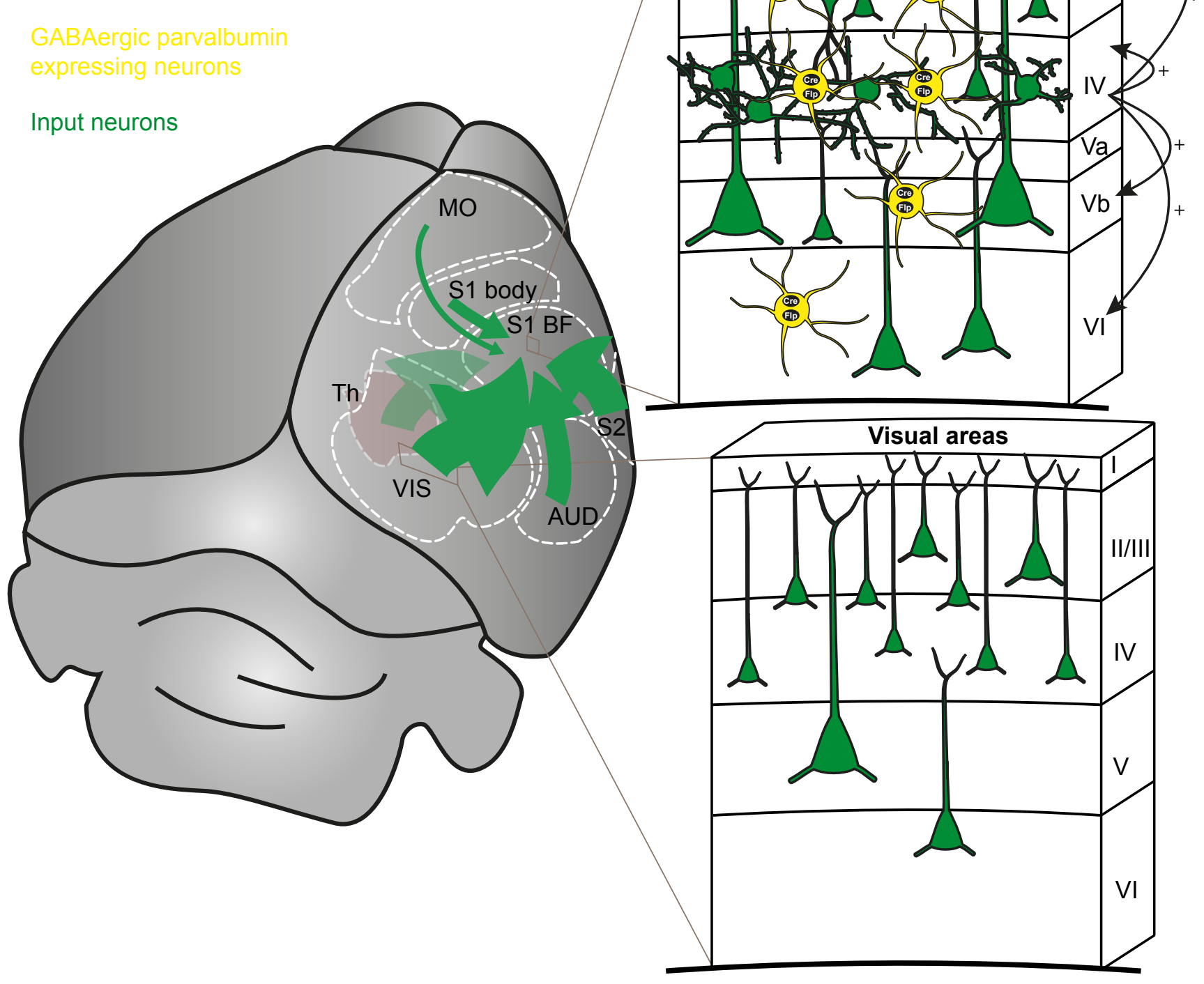

Figure 22: Graphical summary of intersectional RV tracing of GABAergic PV cells in barrel cortex.

In Vgat-Cre/PV-FIp transgenic mice, GABAergic PV cells are molecularly tagged by a co-expression of Creand Flp-recombinase. With a combination of Cre- and-Flp dependent AAVs and RV, we traced the brain-wide inputs to inhibitory PV cells in barrel cortex. Looking at the local connectivity within the barrel cortex, we found that the majority of excitatory input originated from LIV. GABAergic neurons in LI provided inhibitory input to LII/III PV cells. The major sources of long-range input were constituted by visual cortex, secondary somatosensory cortex, auditory cortex and the thalamus.

Abbr.: AUD, auditory cortex; MO, motor cortex; S1 BF; primary somatosensory cortex barrel field; S1 body, primary somatosensory cortex body region; S2, secondary somatosensory cortex; Th, thalamus; VIS, visual cortex 

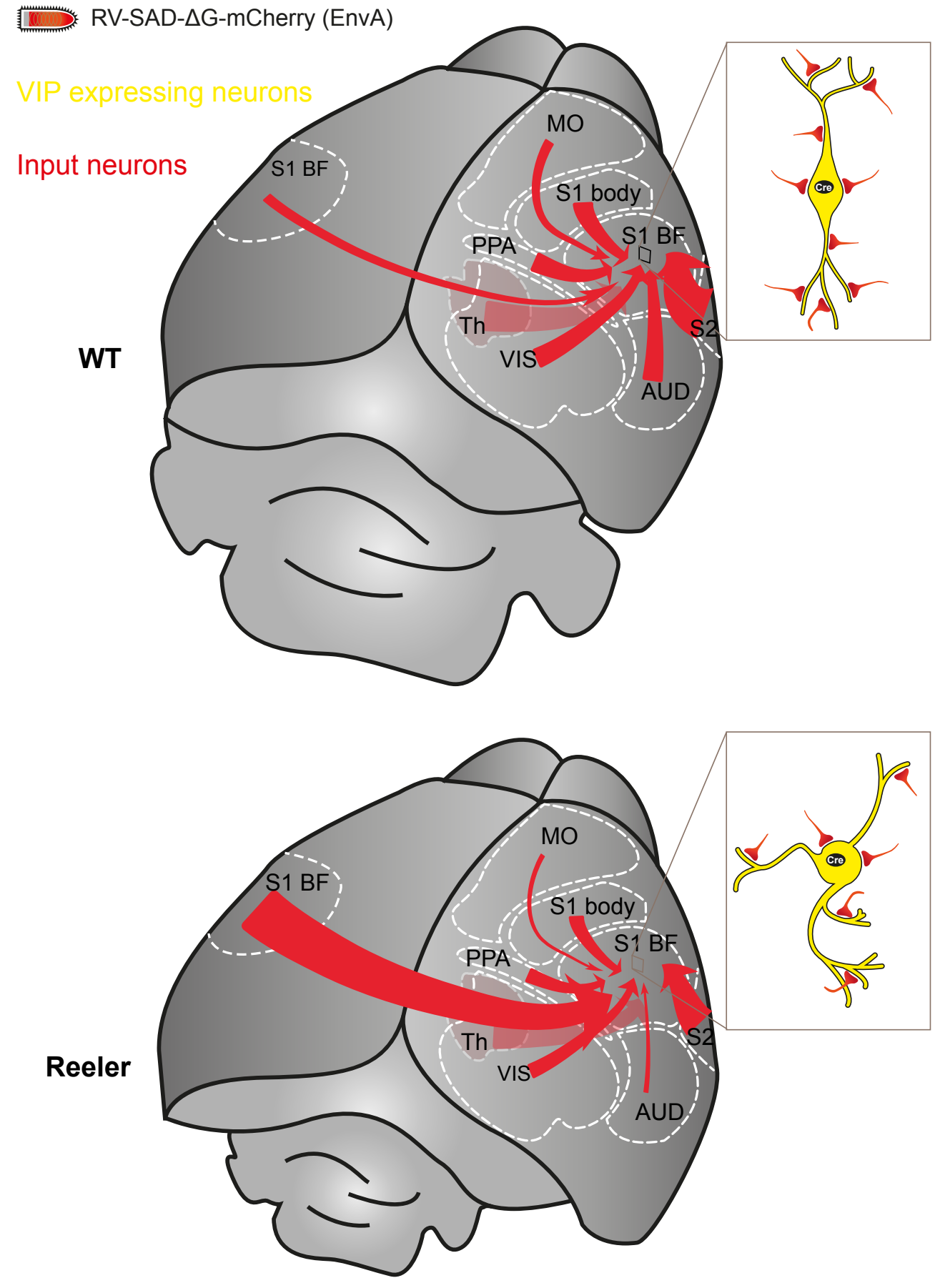

Figure 23: Graphical summary of comparative RV tracing of VIP neurons in the barrel cortex of WT and reeler mice.

In reeler mice, VIP neurons showed no biased distribution towards the upper cortical layers and had a more multipolar than bipolar morphology. By using a combination of a Cre-dependent AAV and RV, we mapped the long-range inputs to VIP neurons. In both genotypes, VIP neurons received input from the same sources but in different proportions. In reeler mice, VIP neurons received considerably less input from other ipsilateral cortical areas but about four-fold more input from the contralateral barrel cortex. The magnitude of subcortical input was the same. 
Abbr.: AUD, auditory cortex; MO, motor cortex; PPA, posterior parietal association area; S1 BF; primary somatosensory cortex barrel field; S1 body, primary somatosensory cortex body region; S2, secondary somatosensory cortex; Th, thalamus; VIS, visual cortex

\subsection{Caveats of RV-tracing}

RV-tracing has been established as the state-of-the-art technique for visualizing brain-wide inputs to a certain cell type. Despite all its advantages (mentioned in introduction), RV-tracing exhibits some inherent limitations that need to be taken into consideration when interpreting the results.

The main error when analyzing long-range input stems from its likely incompleteness. There are two critical determinants for the fraction of inputs RV will label: the number of RV particles infecting a starter cell and the levels of glycoprotein (Callaway and Luo, 2015). The number of initial RV particles in a cell is influenced by the titer of RV and by the abundance of the TVA receptor expression. The levels of glycoprotein need to exceed a critical threshold, in order to reconstitute infectious particles (Callaway and Luo, 2015; Weible et al., 2010). This might happen only in a fraction of starter cells. Although we used oG in both projects, the number of retrogradely labeled cells when tracing PV cells with intersectional constructs was much lower than when tracing VIP cells with Cre-dependent constructs. Therefore, we assume that the requirement for double-dependent recombination of oG exacerbates the build-up of high levels. Moreover, RV is inherently not designed to jump at every synapse (Ghanem and Conzelmann, 2016; Luo et al., 2008). Therefore, this technique can only reveal a hitherto undefined fraction of the whole connectivity scheme.

Considering that RV labels only a fraction of inputs, there might be a bias of RV to label certain inputs more likely than others (Callaway and Luo, 2015). If a population of presynaptic cells establishes more synaptic contacts, their probability of being traced might increase. There could be a vested bias of RV for certain synapses that expose high affinity receptors for glycoprotein. For example, it could be that RV is biased towards cholinergic fibers because one well known interaction partner of the glycoprotein is the nicotinic acetylcholine receptor (Oswald et al., 2017).

On the other hand, there could be fibers that escape tracing because RV avoids their synapses. For example, RV tracing of newborn neurons in the olfactory bulb failed to label any mitral cells, a source of input to granule cells (Deshpande et al., 2013). Similarly, neurons in the mPFC seem to be less likely to take up RV so that a tracing with rAAV2-retro (Tervo et al., 2016) reveals far more mPFC inputs than with RV (Sun et al., 2019). Early studies with wildtype RV have hinted that this virus does not or only poorly transfect synapses from locus coeruleus (Astic et al., 1993; Ugolini, 1995).

Finally, RV tracing does not reveal information about the strength of a connection. Although a high number of presynaptic cells converge on a neuron, only a few of these might provide an input strong enough to activate the neuron to partake in the active network (Cossell et al., 2015). For example input from the anterior cingulate to hippocampal CA3 recruits much more neurons after contextual fear learning (Rajasethupathy et al., 2015). This indicates that anatomical connections can be highly dynamic in their strength. Because RV spreads to presynaptic cells independent of synapse activity, synaptic strength remains an unknown dimension in the RV tracing approach (Ghanem and Conzelmann, 2016). 


\subsection{Control experiments are necessary to assess the cell-type specificity}

All these caveats aside, RV-based tracing is currently the best technique to label monosynaptic inputs to a defined cell class. However, rigorous control experiments are necessary to resolve all doubt that the tracing is indeed cell-type specific.

The first step to guarantee cell-type specificity is the validation of the Cre-expressing mouse line. Recombinase-expression must be restricted to the cells of interest. While in the VIP-Cre mouse line Cre expression is almost fully restricted to VIP positive cells (Prönneke et al., 2015), the PV-Cre line includes about $5 \%$ excitatory cells in its Cre-expressing population. We bred an intersectional mouse line, Vgat-Cre/PV-Flp, to exclude this excitatory population almost completely. Therefore, our mouse lines fulfilled the requirements for cell-type specific tracing.

The second step is to validate if the AAV helper viruses express their proteins only in the presence of recombinases. We utilized two different approaches for our tracing, one using the constructs AAV-Con/Fon-TVA-mCherry and AAV-Con/Fon-oG and the other one using AAV-TVA ${ }^{66 T}$-EGFP-oG. In both cases we investigated if our tracing exposes solely cell-type specific inputs on the local as well on the long-range level. We injected these constructs into BL6 (no site-specific recombinase-expressing wild type) animals followed by RV. In these experiments no RV labeling should occur. However, tracing with AAV-Con/Fon-TVA-mCherry and AAV-Con/Fon-oG resulted in RV labeling at the injection site, but not in distant parts outside of the injection site. If these neurons at the injection site stem from transsynaptic spread, then we would expect scarce labeling in distant areas as well. As this was not the case, we considered the glycoprotein to be expressed strictly recombinase dependent. Local labeling can result from leak of TVA (DeNardo et al., 2015; Do et al., 2016; Faget et al., 2016; Mijamichi et al., 2013; Watabe-Uchida et al., 2012; Weissbourd et al., 2014; Zhang et al., 2016). Low-level leak of TVA, in the absence of site-specific recombinases, allows RV to enter cells due to the high affinity of its EnvA-pseudotyped envelope to the receptor (Mijamichi et al., 2013). These cells display no detectable mCherry signal, making their TVA leak "invisible". Upon RV uptake they appear as retrogradely labeled cells leading to an overestimation of the true count of presynaptic cells. However, we expect that proportions of local inputs among layers are unaffected by this error because the leak seems to be a random phenomenon. We emphasize the importance of conducting additional, independent experiments to verify local connections revealed by RV tracing, as we have done exemplarily with glutamate uncaging for the LI-input.

Tracing with AAV-TVA ${ }^{66 T}$-EGFP-oG caused neither local nor distant leak. The 66T-mutation in the TVA sequence reduces the affinity of EnvA-pseudotyped RV to the receptor and thus a much higher density of receptors is required at the cell surface to permit RV-entry into the cell (Mijamichi et al., 2013). This high density can only be reached in recombinase expressing cells. In consequence, this construct allows a completely cell-type specific tracing.

Previous studies using this mutated construct have raised the concern that the obliteration of leak expression is payed at the expense of tracing efficiency because of a lower number of starter cells and a lower number of initial RV particles entering (Mijamichi et al., 2013; Weissbourd et al., 2014). We witnessed a much higher number of starter cells than in another study tracing VIP cells (Wall et al., 2016) and our ratio of presynaptic cells to starter cells was comparable to regular TVA based tracing 
(Mijamichi et al., 2013; Wall et al., 2016). Most likely use of oG in our construct boosted transsynaptic labeling efficiency (Kim et al., 2016).

\subsection{Brain-wide inputs to GABAergic PV cells in mouse barrel cortex}

The initial attempt to trace brain-wide inputs to inhibitory PV cells was hampered by the fact that the population of cells labeled in the PV-Cre line includes a small number of excitatory cells (Madisen et al., 2015; Wall et al., 2010 and own observation). We followed an intersectional strategy, which proved to be very successful in abolishing the excitatory PV population. In Vgat-Cre/PV-Flp mice the co-expression of two site-specific recombinases, Cre and Flp, is driven by the parvalbumin- and the inhibitory neuron specific Vgat-promotor. This mouse line allowed to target GABAergic PV neurons with much higher specificity than the PV-Cre line. Using intersectional viruses in combination with this mouse line, we mapped local and long-range inputs to GABAergic PV cells in mouse barrel cortex.

\subsection{Local inputs to PV cells}

The local input to PV neurons outweighed the long-range input by about tenfold. In fact, $92 \%$ of total inputs to PV cells originated from the ipsilateral barrel cortex. This number is a slight overestimation because of the confounder caused by leak of TVA and subsequent direct transduction of cells by RV at the injection site, as discussed above. Nevertheless, this proportion is comparable to the $79 \%$ of inputs within barrel cortex to LV cells (DeNardo et al., 2015). Barrel cortex seems to be a highly interconnected area in which local inputs dominate. LIV provided the majority of inputs to PV neurons within barrel cortex, also being the hub of excitatory inputs. Among inhibitory inputs we focused on the fraction of inputs from LI. Although there are relatively few cells in LI, they made up about $5 \%$ of all local inputs to PV cells, a surprisingly large fraction. We could show that $\mathrm{LI}$ cells preferentially target $\mathrm{PV}$ cells closer to the LI border. Our approach using glutamate uncaging did not allow us to identify the type of presynaptic LI cell (Schuman et al., 2018). Data from multiple simultaneous cell recordings in mouse visual cortex suggest that both single bouquet cell-like (SBC-like) and elongated neurogliaform cells (ENGFs) maintain connections to LII/III PV cells (Jiang et al., 2015). The postsynaptic excitatory targets of PV cells innervated by LI interneurons are pyramidal neurons in LII/III and LV (Lee et al., 2015). This disinhibitory motif from LI IN->LII/III PV->LII/III/V pyramidal neurons could provide the anatomical basis for a gating mechanism, releasing inhibition of excitatory cells upon cortico-cortical input (De Marco García et al., 2015), higher-order thalamic input (Cruikshank et al., 2012) or other subcortical inputs (Letzkus et al., 2011) arriving in LI. That this connection has behavioral relevance has been shown for the auditory cortex, where disinhibition of LII/III pyramidal neurons by the LI-LII/III PV cell connection mediated associative learning (Letzkus et al., 2011). Similarly, it has been speculated that LI interneurons affect the firing rate of $\mathrm{PV}$ cells in barrel cortex during a whisker stimulus detection task (Sachidhanandam et al., 2016). 


\subsection{Long-range input to PV cells}

When analyzing the long-range input to PV cells in the barrel cortex, we found that about two thirds of the total long-range input originated from cortical and the remnant third from subcortical sources. Cortical input originated mainly from other sensory areas like visual and auditory cortex, as well as S2, indicating a direct role of PV neurons in crossmodal sensory integration.

A previous study has already traced long-range inputs to PV cells in the barrel cortex using the PV-Cre line (Wall et al., 2016). We found a qualitatively almost identical set of inputs but the relative contributions from input areas were different. We saw a higher proportion of long-range inputs from visual cortex and a smaller from motor cortex. Moreover, input from the contralateral hemisphere was almost absent in our study while it was a major input source in Wall et al. (2016). These differences must not necessarily be due to the potential contamination with excitatory cells in their PV-Cre line. Instead, already different volumes and titers of viruses could cause substantial differences in input maps (Guo et al., 2015).

Our layer-dependent analysis of cortical long-range input revealed that LIV of visual cortex contained a much higher number of projection neurons than upper or deep layer compartments. Research on the interconnectivity of primate cortical areas led to the dogma that LIV neurons receive thalamic and lower-order area input but do not maintain feedforward projections, fostering a picture of LIV as an input layer (Felleman and Van Essen, 1991). Instead, LII/III has been ascribed the role of the major source of projections to higher order areas.

It is important to keep in mind that we ascribed somata of retrogradely labeled cells to LIV, based on the higher cell density of this layer discernable in nuclear stains. In our experiments, the projection neurons in visual cortex were often localized at the border of LIII and LIV. This layer border is on the one hand not very precisely delineable (Staiger et al., 2015), and on the other hand cells can appear in a certain layer but actually have a closer functional similarity to cell types in a neighboring layer (Narayanan et al., 2015). Therefore, it is possible that some of the cells we ascribed to LIV are deep LIII projection neurons.

To check if actual LIV cells in visual cortex project to barrel cortex, we performed anterograde tracing in the Scnn1a-Tg3-Cre mouse. Previous studies have shown that LIV projection neurons in visual cortex labeled in this mouse line send sparse ramifications into barrel cortex (Harris et al., 2018). Our experiment showed that actual LIV neurons do target PV neurons in barrel cortex in all layers. However, this mouse line does not label exclusively LIV cells but also a few LV/VI cells. Although we expect that this minor complement of LV/VI neurons maintains projections to barrel cortex, too, it is highly unlikely for them to be exclusively (if at all) responsible for the innervation of PV cells we observed in barrel cortex.

Because visual cortex LIV receives strong, direct input from the lateral geniculate nucleus, we speculate that this connection from LIV to barrel cortex might serve the fast propagation of visual information. We are only aware of one study looking at the anatomical projections from visual cortex to barrel cortex in mouse (Massé et al., 2016). The authors report that $38 \%$ of input from V1 to S1 originated from LII/III but only $22 \%$ from LIV, the same proportion as LV and VI. Visual information is integrated into whisker based perception, indicated by the results that simultaneous visual and whisker stimulation increases evoked sensory responses in barrel cortex (Sieben et al., 2013). It could be that 
the LIV neurons in visual cortex innervate PV cells more abundantly or that LIV neurons connect with more synapses on PV cells (or any cell) so that they are more likely traced by RV. A PV cell specific connection could suggest that visual cortex refines tactile representations by sharpening sensory responses upon multisensory stimulation. The physiological properties and relevance of this projection from visual to barrel cortex remain to be investigated further.

Subcortical input consisted almost exclusively of thalamic projections. The main thalamic input was from the VPM, the primary relay nucleus of whisker-related sensory information to the barrel cortex. Although PV neurons do not seem to receive more thalamic input per cell than other interneuron types (Wall et al., 2016), they seem to be recruited the strongest (Cruikshank et al., 2010; Ji et al., 2015). In addition, they are highly abundant in the major thalamorecipient LIV, so that they have been ascribed the role as the major mediator of feedforward inhibition (Feldmeyer et al., 2018). This role is supported by our finding of a high proportion of thalamic inputs, comparable to proportions reported for excitatory cells in barrel cortex (DeNardo et al., 2015).

In summary, we showed that GABAergic PV cells receive, in addition to a dense local innervation, long-range inputs, mainly from other sensory areas and the thalamus. We focused on two circuit motifs. On the one hand, the local connection from LI interneurons to LII/III PV cells, suggesting a disinhibitory circuit for precise gating of information processing. On the other hand, a novel long-range connection from the thalamorecipient LIV in visual cortex to barrel cortex, a possible highway for fast relay of visual information. We introduced intersectional rabies virus tracing as a novel tool to target subpopulations of neurons and used it to isolate the inhibitory population of PV expressing cells This technique will allow to further dissect the circuits of neuronal subtypes because it can be applied to any combination of cell types for which Cre/Flp mouse lines are available (He et al., 2016; Madisen et al., 2015).

\subsection{Brain-wide inputs to VIP cells in WT mouse barrel cortex}

Before discussing the differences in long-range inputs to VIP cells in barrel cortex of WT and reeler mice, the results for WT alone will be discussed to set them into the context of what is known about their inputs already.

Long-range inputs to VIP cells in barrel cortex originated $80 \%$ from cortical and $20 \%$ from subcortical areas. Cortical input was dominated by input from the secondary somatosensory cortex, posterior parietal association area, auditory cortex, visual cortex and motor cortex. Therefore, VIP neurons in barrel cortex are directly recruited by circuits for sensory and motor integration. The connection from motor cortex to VIP neurons in S1 has already been dissected before (Lee et al., 2013). Motor cortex strongly activates VIP neurons in S1 resulting in inhibition of SST neurons and subsequent disinhibition of pyramidal neurons to open a window for integration of whisker related movements.

Subcortical input was dominated by the thalamus, especially the VPM, which constituted half of the total subcortical input, and the PO. Direct thalamic input onto VIP neurons primarily targets their distal dendrites and might engage them in feedforward inhibitory actions (Audette et al., 2017; Sohn et al., 2016; Staiger et al., 1996b). PO input to VIP neurons is a vital component for eliciting synaptic 
plasticity at excitatory synapses of pyramidal neurons in LII/III, again by a suggested disinhibitory action (Williams and Holtmaat, 2019).

The previous study tracing long-range inputs to VIP cells in barrel cortex reported a qualitatively similar array of inputs but with minor differences in their proportions (Wall et al., 2016). For example, about $30 \%$ of total inputs to VIP cells in barrel cortex originated from the VPM, about 2.5-fold higher than our fraction. In comparison, the value for thalamic input to VIP neurons in visual cortex with about 14\% was fairly similar to our data (Zhang et al., 2016). The factor that could account for this difference might be their lower number of starter cells, being about 20 times lower than in our study. Therefore, Wall et al. (2016) looked at a fairly small population of VIP cells, in which case phenomena of convergence and divergence of presynaptic fibers come into effect more severely than in our bigger sample. As a result, the ratio of presynaptic to starter cells is easily distorted. Thus, our proportions might be more representative for the entirety of the VIP population.

Proportions of cortical inputs deviated from our results as well. Wall et al. (2016) reported more input from motor cortex in contrast to less input from visual and auditory cortex. This difference could be provoked by the location of starter cells. In our study they were located in the caudal part of barrel cortex and in Wall et al.'s in the rostral part.

In general, our study provides a much finer-grained breakdown of the cortical inputs, and especially from subcortical sources than Wall et al. (2016). Because of our high number of starter cells and our very efficient tracing, we could label many more inputs per brain. Thus, we yielded sufficiently high numbers of cells for statistical analysis already on the level of subareas, while Wall et al. (2016) had to lump subareas together into global areas to have statistically useful values.

In conclusion, this study provides a comprehensive map of the brain-wide, monosynaptic inputs to VIP cells in the barrel cortex. Although a previous study has mapped these inputs to VIP cells in barrel cortex, our study entertains with a higher number of traced cells and a more detailed mapping, and thus provides a more complete picture of the inputs.

\subsection{Comparison of brain-wide inputs to cortical GABAergic neurons}

Three main types of cortical GABAergic cells, PV, SST and VIP expressing neurons, vary in laminar distribution, morphology and electrophysiological properties. Hence they have been ascribed distinct functions in the cortical circuitry (Tremblay et al., 2016). Therefore, it seemed imperative that these neurons do not share the same kind of inputs because the inputs to a cell profoundly influence its functional output. At the beginning of this PhD thesis we asked the central question, if GABAergic neurons in the mouse barrel cortex receive different sources of brain-wide input. Initially, VIP neurons were suspected to act as the primary integrators of long-range input (Fu et al., 2015; Lee et al., 2013; Zhang et al., 2014). Their activation by these sources then leads to a temporary surge in excitation due to disinhibitory mechanism (Fu et al., 2014; Jackson et al., 2016; Kuchibhotla et al., 2016; Lee et al., 2013; Pi et al., 2013; Zhang et al., 2014). Several studies have comparatively assessed the brain-wide 
inputs to cortical inhibitory cells in frontal (Zhang et al., 2016), auditory (Nelson and Mooney, 2016) and visual cortex (Zhang et al., 2014, 2016) and, most extensively, in barrel cortex (Wall et al., 2016). Together with the current study tracing the isolated classes of VIP and PV cells in barrel cortex and the studies tracing LI neurons in auditory cortex (Abs et al., 2018), Erb4 expressing neurons in frontal cortex (Choi and Callaway, 2011), PV neurons in medial prefrontal cortex (Delevich et al., 2015), and VIP neurons in the visual cortex (Fu et al., 2014), there is substantial literature about the brain-wide inputs to cortical GABAergic cells. All the studies point to the same conclusion: Each GABAergic neuron in a certain area receives input from the same sources!

These sources constitute input from virtually all other neocortical areas. Especially the sensory areas are highly interconnected suggesting that interneurons play a crucial role in the cross-modal integration of information. Subcortical sources constitute lower and higher order thalamic nuclei. The most numerous inputs are from the primary relay nuclei (VPM, dorsal and lateral geniculate nucleus). Also the basal forebrain is a consistent input source. It is an important neuromodulatory center that can profoundly influence cortical processing (Lin et al., 2015).

There are differences in the proportion of inputs from these sources, but they are too small or too inconsistent to be significant. Nevertheless, one study has detected a difference in the input magnitude among GABAergic cells (Wall et al., 2016). The authors report that VIP cells received more long-range input per individual cell than SST or PV neurons, again suggesting a role for them as primary integrators of long-range input. However, one fact needs to be taken into consideration. Their number of VIP starter cells is much smaller than their number of PV or SST starter cells. Considering that presynaptic cells could diverge and innervate multiple starter cells at once, a higher number of starter cells coincides with fewer inputs detected per starter cell, hence a lower input magnitude. Thus, this result could be an artifact based on their sample selection, which has not been addressed in their paper.

Excitatory cells have been traced in a similar way. LII/III, LV and LVI cells in barrel and prefrontal cortex (DeNardo et al., 2015), LV projection neurons in visual cortex (Kim et al., 2015), and to excitatory neurons in visual, barrel, auditory and frontal cortex (Zhang et al., 2016) as well as single cell RV-tracing studies of pyramidal neurons in visual cortex (Marshel et al., 2010; Rancz et al., 2011; Velez-Fort et al., 2014; Wertz et al., 2015). They receive input from the same sources as inhibitory cells which is not surprising because inhibition follows excitation (Isaacson and Scanziani, 2011). However, for excitatory neurons there are stronger differences in the proportions of inputs from different sources. For example, LV cortico-cortical neurons receive less thalamic input than LV cortico-subcortical neurons (Kim et al., 2015). Still, this fact points to the same conclusion that all neurons in a cortical area receive the same qualitative input.

There are two possibilities how to explain this rather surprising conclusion. First, the differences in inputs are too subtle to be detected with the current RV-tracing approaches. Neuronal subtype specification based on these global molecular markers could be too broad, cortical inputs could vary in their laminar distribution or RV tracing does not label enough presynaptic cells to unveil differences. Improvement of RV-tracing tools could aid in the detection of minute differences. Intersectional RV tracing can prove valuable to investigate more clearly defined subgroups of neurons. Reducing leak expression can improve the off-target tracing of neurons (Mijamichi et al., 2013). Incorporating new variations of the rabies glycoprotein can improve the efficiency of the tracing (Kim et al., 2016). These technical improvements have to go hand in hand with a detailed analysis of the traced areas and layers 
aided by automation of cell counting and atlas referencing to handle these large numbers of data (Fürth et al., 2017).

Second, the function of a neuron is not encoded in the types of inputs. The same input can have very different effects on neurons with different intrinsic properties. Maybe the heterogeneity of cortical GABAergic neurons emerged as a solution to generate different outputs from similar inputs. Furthermore, the strength of an input might be a crucial parameter for the activation of cell types, weighing more important than the source. Therefore, anatomical tracing techniques do not allow to predict the functional interactions of brain-wide networks. Instead, they can provide a basis for hypotheses about the impact of a connection. The connection itself needs to be investigated with measurements of connection strength and in context of the intrinsic properties of the receiving neuron.

\subsection{Comparison of brain-wide inputs to VIP neurons of WT and reeler mice}

The reeler mutant is an excellent model system to study the significance of layers for cortical processing (Guy and Staiger, 2017). We asked the question if VIP neurons in reeler mice are embedded in the same long-range circuits as in WT mice. VIP neurons have a particular laminar bias towards upper layers, which is completely dispersed in reeler. Therefore, we expected that such a change in cellular arrangement coincides with alterations in the afferent circuitry. We used retrograde RV-tracing to comparatively map the brain-wide inputs to VIP neurons in WT and reeler.

\subsection{VIP neurons receive less ipsilateral and more contralateral cortical long-range input}

We found that VIP neurons in reeler receive input from the same sources as in WT, however, we found profound quantitative differences. The most prominent findings were that VIP neurons in reeler receive less input per cell and the proportions of ipsilateral vs. contralateral inputs is fundamentally shifted.

The lower ratio of presynaptic cells to starter cells (input magnitude) could result either from an actual lower number of inputs or from a consistently lower efficiency in RV tracing in reeler. The structure of synaptic boutons or the innervation pattern of postsynaptic targets is not altered in reeler (Prume et al., 2018). Therefore, we have no indication that RV tracing could be inherently less efficient in reeler mice and can more confidently attribute the lower input magnitude in reeler VIP neurons to a smaller number of long-range inputs.

Three factors need to be discussed in the light of a lower input magnitude. First, subcortical input magnitude was not (significantly) reduced. Second, the input magnitude for contralateral inputs was even higher in reeler. Third, the reduced input magnitude was entirely due to a smaller number of ipsilateral long-range inputs. What do these findings mean for the initial question if VIP neurons in the reeler cortex are embedded in the same long-range circuits? 
The thalamic input, the major subcortical input, arises from a properly developed structure and projects to a mislaminated cortex. The cells in the thalamus are not affected by the reelin mutation (Wagener et al., 2010). Previous studies have shown that thalamic fibers from the VPM reach their malpositioned postsynaptic targets in the reeler cortex although they take an unusual trajectory reaching $\mathrm{LI}$ before bouncing back to plunge down on the target cells (Caviness and Frost, 1983; Guy et al., 2016; Harsan et al., 2013; Wagener et al., 2015). These studies together with the current results support the notion that fibers from subcortical structures find their ectopic targets in reeler cortex. Therefore, the malposition of the postsynaptic VIP cells seems not to affect their capacity to be integrated into functional long-range circuits.

Cortical long-range input showed clear quantitative differences between WT and reeler. About four times more neurons in the contralateral barrel cortex innervated VIP cells in reeler than in WT. This hints at a higher callosal connectivity between homotopic areas in reeler. This could be a cell-type specific effect so that only VIP neurons collect more callosal input on the expense of other neurons. Then the overall number of callosal projection neurons (CPNs) could be the same in both genotypes. However, it is more likely that there is an overall higher number of CPNs in reeler for two reasons. Most retrogradely labelled cells in WT were in LII/III. This cell population is more numerous in reeler compared to WT (Polleux et al., 1998; Wagener et al., 2010). This could be a hint that there are more CPNs in reeler. Furthermore, tracing data in WT revealed that all three types of GABAergic neurons receive very similar input. Although these results cannot be directly translated to reeler, it is more likely that also in the mutant GABAergic neurons integrate similar inputs to preserve the balance of inhibition. Hence, there have to be more fibers to innervate all cell types equally. Measuring the thickness of the corpus callosum with magnetic resonance imaging or related imaging methods could already point to a difference in the number of fibers crossing hemispheres. Although previous studies have investigated the size of the reeler brain and the connectivity of fiber tracts (Badea et al., 2007; Harsan et al., 2013), the architecture of the corpus callosum has not been comparatively assessed. Injection of a non-cell-type specific retrograde tracer like cholera toxin B or RV-SAD $\triangle$ G-EGFP (CSV-G), which are taken up by axonal terminals, would allow assessing the global number of CPNs independently of the postsynaptic cell type.

The excess in callosal inputs to VIP cells in reeler went hand in hand with a shortage of ipsilateral cortical inputs. Because numbers of subcortical inputs were similar in reeler and WT despite the malposition of the postsynaptic target, we assume that rather the malposition of cortico-cortical projection neurons exacerbates their ability to establish long-range connections than the malposition of the VIP cells. Despite this reduced ipsilateral input, we noticed that the proportion of total cortical input was roughly the same between WT and reeler. Therefore, we speculate that the reduced ipsilateral input was compensated by the increase in contralateral inputs.

About $80 \%$ of CPNs in rodents are located in LII/III and $20 \%$ in LV with a few in LVI (Fame et al., 2011; Leyva-Díaz and López-Bendito, 2013). LII/III CPNs are derived from intermediate progenitors in the subventricular zone and born at E15.5, later than all the other projection neuron subtypes (Molyneaux et al., 2009). Their axons cross the midline around the time of birth but only start to grow into the grey matter during the first postnatal week (Wise and Jones, 1976). They have local axonal branches on the ipsilateral hemisphere in addition to their axonal branch across the midline. On both hemispheres they connect with specificity to LII/III, LV and a subset of LVI neurons omitting LIV (Petreanu et al., 2007). However, the cell-type specific ipsilateral connections mature earlier indicating that 
ipsilateral circuits are already established before the contralateral projections are getting integrated (Petreanu et al., 2007). Callosal projections are transient during development. They are very numerous during early postnatal stages and are reduced later (Innocenti and Price, 2005; Ivy et al., 1984; Wise and Jones, 1976). In the first two to three postnatal weeks, developmental pruning of axons allows for the establishment of specific connections (O'Leary, 1992; O'Leary et al., 1981). For CPNs there is clear evidence that axonal pruning is a mechanism to eliminate axonal synapses of callosal neurons (Innocenti, 1981; Price and Blakemore, 1985). This process of developmental pruning seems to depend on electrical activity leaving only synapses in used circuitries intact (Zhang and Poo, 2001). Maybe in reeler, the degeneration of callosal axons happens to a lesser degree because the callosal input is needed to compensate for the lack of ipsilateral input. This would allow to balance out the strength of the multiple afferent systems (Caviness and Rakic, 1978).

The imbalance of contra- vs. ipsilateral inputs in reeler has not been noticed before although callosal projections have been investigated. CPNs have been confirmed as a pool distinct from subcortical projecting neurons in reeler (Imai et al., 2012). They project to the homotopic contralateral area like in WT, despite that fact that they are located in deeper parts of the cortex (Caviness Jr. and Yorke Jr., 1976; Steindler and Colwell, 1976). Similarly, no differences have been observed in the capacity of other cortico-cortical or corticofugal projection neurons to innervate their target cells. The proper establishment of the projection from entorhinal cortex to the hippocampal formation (Stanfield and Cowan, 1979), the projection from piriform cortex to the medial prefrontal cortex, the amygdala and the entorhinal cortex (Diodato et al., 2016) as well as the projection from the motor cortex to the thalamus (Diodato et al., 2016) all demonstrate the ability of ectopic projection neurons to find their long-range targets despite the absence of layers. However, these studies did not assess the magnitude of these inputs but just their qualitative nature. It seems that malpositioned neurons do find their long-range targets but not in the same numbers.

How this imbalance affects behavior can only be speculated about at the moment. A fact that cannot be assessed by RV-tracing is the strength of synaptic connections. It might be the case that ipsilateral connections in reeler are stronger and contralateral weaker. C-fos staining after whisker stimulation has shown that presumably callosal LII/III fate neurons are activated to a lesser extent in reeler than in WT although they are more numerous in reeler (Wagener et al., 2015). This could hint that these neurons do not contribute as much to the network activity in reeler as they do in the wild type. This study also confirmed the aptitude of reeler mice for tactile exploration tasks as they use their whiskers for tactile exploration just like WT mice (Wagener et al., 2015). Therefore, reeler mice undergoing behavioral tasks that require bilateral whisker integration like the gap detecting task (Chaudhary and Rema, 2018), could clarify if reeler mice rely more on bilateral information than WT mice. Given this premise, blocking the contralateral input would impair reeler more than WT mice.

\subsection{The proportions of cortical inputs are preserved in reeler with a few exceptions}

After assessing the global balance between ipsi-and contralateral inputs we focused on differences among individual areas between the two genotypes. Overall, the same areas projected to VIP neurons 
in WT and reeler. Comparison of the proportion of ipsilateral inputs, however, highlighted three areas that send different proportions of inputs to VIP neurons in the barrel cortex: Orbital area and primary auditory cortex constituted a smaller proportion of inputs in reeler, the VPL constituted a higher proportion of inputs in reeler.

Could these differences have functional implications? It is difficult to predict a functional consequence based on the alteration of a single connection in a complex circuit because neural networks might have mechanism of plasticity to ensure proper function with various circuit arrangements. The reduced connection from primary auditory cortex to VIP neurons in the barrel cortex might be easier to assess than that from a higher order area because it might compromise the integration of auditory signals in the somatosensory network. Indeed, the auditory cortex fuels information about sound to the somatosensory cortex to be integrated into tactile processing (Lemus et al., 2010; Maruyama and Komai, 2018). Hence, the cross-modal interaction of sound and touch might be impaired in reeler mice. Behavioral experiments compelling the animal to integration of sound into tactile perception might be a starting point to study the functional significance of a diminished input from primary auditory cortex to barrel cortex.

Noteworthy is also the non-significantly but slightly higher fractions of input from the limb and trunk somatosensory regions in reeler. This is in contrast to all the other ipsilateral cortical areas, which sent a smaller number of projections to the VIP neurons in the barrel cortex of reeler mice. A fundamental principle of long-range connectivity is the preferential interconnectivity of areas with similar function (Goulas et al., 2017). Maybe in reeler, this principle is even more prominent and the individual sensory areas are more strongly connected within each other but less strongly connected to other sensory areas. This idea could also help explaining the higher input from the VPL. The VPL is the primary thalamic relay nucleus for somatosensory information from the body (limbs and trunk). It receives input from the spinal cord and then projects to body regions of the primary somatosensory cortex medial of the barrel field. The higher input fraction from VPL to VIP neurons in barrel cortex of reeler mice could reflect a less strict somatotopy in these mice that is compensated by stronger interconnectivity of the somatosensory areas.

It is necessary to mention at this point that an input from VPL does not necessitate the presence of starter cells outside of the barrel cortex in body regions of primary somatosensory cortex. We never detected starter cells outside of barrel cortex on our sections. Previous tracing to inhibitory neurons in barrel cortex have reported a VPL input, too, despite the restriction of starter cells to the barrel cortex (Wall et al., 2016). Therefore, we can assume that VPL projects sparsely to barrel cortex and the input is not a result of mistargeted injections.

\subsection{Distribution of cortical projection neurons in reeler}

We looked at the distribution of retrogradely labeled projection neurons across the cortical depth. Previous studies have raised the concept that different cortical areas have different migratory patterns in the absence of reelin. Rostral areas show closer similarity to the WT formation despite dispersion of layer-fated neurons, medial areas show a high degree of disorganization and caudal areas show elements of inversion (Boyle et al., 2011; Dekimoto et al., 2010; Pielecka-Fortuna et al., 2014; Wagener 
et al., 2010). Therefore, we wanted to investigate if the distribution of projection neurons is different between WT and reeler, and if the distribution in reeler matches the known patterns of cellular disarrangement.

Considering that reeler mice lack layers, the distribution of ipsilateral projection neurons was surprisingly similar between the two genotypes but there was a slight trend for neurons in reeler to be closer to the pial surface. Therefore, ipsilateral projection neurons were arranged similarly as in WT and did not follow the general patterns of dispersion observed in reeler. These results cannot be explained if we believe that neuronal fate is the only determinant for establishment of ipsilateral long-range connections. Instead it seems that the depth of the neuron in the cortex is an additional factor influencing the neuron's ability to form a functional long-range connection. This idea is supported by the fact that overall less neurons in reeler manage to establish ipsilateral long-range connections. Only those neurons with the proper fate that end up in the correct position contribute to the long-range circuits, while those neurons with the proper fate but the wrong position fail to contribute. Perhaps a signaling molecule other than reelin builds up a gradient across the cortex to influence the axonal outgrowth of neurons. Maybe this signaling function is still intact and recruits the neurons in the proper depth to become longrange projection neurons.

The result for ipsilateral projection neurons was in striking contrast to the one for CPNs. They exhibited an eye-catching difference in the distribution between WT and reeler. In WT, projection neurons were predominantly located in the upper third of the cortex corresponding to LII/III. In reeler, projection neurons were predominantly located in the lower two thirds of the cortex. For WT this distribution was expected because the majority of callosal projection neurons ( $80 \%)$ resides in LII/III (Fame et al., 2011; Leyva-Díaz and López-Bendito, 2013). Previous reports on the distribution of callosal neurons in reeler also noticed that they are preferentially located in deeper parts of the cortex, matching our results (Caviness Jr. and Yorke Jr., 1976; Imai et al., 2012; Steindler and Colwell, 1976).

It could be possible that in reeler mice neurons with the fate of LII/III still maintain their fate as callosal projection neurons but just do not migrate to their destined position. The fact that LII/III-fated cells, labeled by the LII/III specific marker Rgs8, occur in higher numbers in reeler could point to the fact that more of these callosal neurons persist in reeler after cortical maturation (Wagener et al., 2010). However, these LII/III fated cells in reeler are dispersed rather evenly across the cortical depth (Wagener et al., 2010), while the CPNs omit the upper third. Thus, the Rgs8 population might not match the population of CPNs entirely. Instead, neurons positive for the marker Satb2 could comprise the population of CPNs and might be more numerous in reeler. Satb2 is a marker for CPNs and its knock-out causes an almost complete absence of the corpus callosum. (Alcamo et al., 2008; Britanova et al., 2008; Greig et al., 2013; Leyva-Díaz and López-Bendito, 2013). Furthermore, Satb2 neurons occur preferentially in deeper parts of the cortex in reeler mice (Alifragis et al., 2006). Because in our tracing CPNs were mainly located in deeper parts of the cortex, too, this is a further hint that an excess of Satb2 neurons in reeler might be responsible for the stronger contralateral innervation of VIP cells. Combining the RV-tracing experiments with stainings for layer fate markers like Rsg8 and Satb2 could clarify which type of neurons make up CPNs in reeler.

In conclusion, this study presented a comprehensive map of the brain-wide inputs to VIP cells in barrel cortex of reeler mice, which was compared to WT. While the cortical inputs to VIP cells in 
reeler exhibited a totally different balance of ipsi-vs. contralateral inputs, subcortical inputs remained in the same proportions. The bottom-up flow of sensory information seems to run in preserved celltype specific projections despite the ectopic position of the cortical targets. The cortical circuitry for the processing of sensory information, however, is altered in the absence of layers. Based on our results we speculate that a smaller number of ipsilateral connections is compensated by a higher number of callosal connections between homotopic areas. If this putative adaption is specific to VIP neurons and what its behavioral relevance is will be exciting questions to answer in future experiments. 


\section{REFERENCES}

Abs, E., Poorthuis, R.B., Apelblat, D., Muhammad, K., Pardi, M.B., Enke, L., Kushinsky, D., Pu, D.-L., Eizinger, M.F., Conzelmann, K.-K., et al. (2018). Learning-Related Plasticity in Dendrite-Targeting Layer 1 Interneurons. Neuron 100, 684-699.

Adesnik, H., Bruns, W., Taniguchi, H., Huang, Z.J., and Scanziani, M. (2012). A neural circuit for spatial summation in visual cortex. Nature 490, 226-231.

Alcamo, E.A., Chirivella, L., Dautzenberg, M., Dobreva, G., Fariñas, I., Grosschedl, R., and McConnell, S.K. (2008). Satb2 Regulates Callosal Projection Neuron Identity in the Developing Cerebral Cortex. Neuron 57, 364-377.

Alifragis, P., Junek, S., Tarabykin, V., Gruss, P., Britanova, O., and Jones, K. (2006). A novel mode of tangential migration of cortical projection neurons. Dev. Biol. 298, 299-311.

Aronoff, R., Matyas, F., Mateo, C., Ciron, C., Schneider, B., and Petersen, C.C.H. (2010). Long-range connectivity of mouse primary somatosensory barrel cortex. Eur. J. Neurosci. 31, 2221-2233.

Ascoli, G.A., Alonso-Nanclares, L., Anderson, S., Barrionuevo, G., Benavides-Piccione, R., Burkhalter, A., Buzsáki, G., Cauli, B., Defelipe, J., Fairén, A., et al. (2008). Petilla terminology: nomenclature of features of GABAergic interneurons of the cerebral cortex. Nat. Rev. Neurosci. 9, 557-568.

Astic, L., Saucier, D., Coulon, P., Lafay, F., and Flamand, A. (1993). The CVS strain of rabies virus as transneuronal tracer in the olfactory system of mice. Brain Res. 619, 146-156.

Atallah, B. V., Bruns, W., Carandini, M., and Scanziani, M. (2012). Parvalbumin-Expressing Interneurons Linearly Transform Cortical Responses to Visual Stimuli. Neuron 73, 159-170.

Audette, N.J., Urban-Ciecko, J., Matsushita, M., and Barth, A.L. (2017). POm Thalamocortical Input Drives Layer-Specific Microcircuits in Somatosensory Cortex. Cereb. Cortex 1-17.

Badea, A., Nicholls, P.J., Johnson, G.A., and Wetsel, W.C. (2007). Neuroanatomical phenotypes in the Reeler mouse. Neuroimage 34, 1363-1374.

Baskerville, K.A., Schweitzer, J.B., and Herron, P. (1997). Effects of cholinergic depletion on experience-dependent plasticity in the cortex of the rat. Neuroscience 80, 1159-1169.

Bayraktar, T., Welker, E., Freund, T.F., Zilles, K., and Staiger, J.F. (2000). Neurons immunoreactive for vasoactive intestinal polypeptide in the rat primary somatosensory cortex: morphology and spatial relationship to barrel-related columns. J. Comp. Neurol. 420, 291-304.

Beierlein, M. (2003). Two Dynamically Distinct Inhibitory Networks in Layer 4 of the Neocortex. J. Neurophysiol. 90, 2987-3000.

Bock, H.H., and May, P. (2016). Canonical and Non-canonical Reelin Signaling. Front. Cell. Neurosci. 10, 1-20.

Bortone, D.S., Olsen, S.R., and Scanziani, M. (2014). Translaminar inhibitory cells recruited by layer 6 corticothalamic neurons suppress visual cortex. Neuron 82, 474-485.

Boyle, M.P., Bernard, A., Thompson, C.L., Ng, L., Boe, A., Mortrud, M., Hawrylycz, M.J., Jones, A.R., 
Hevner, R.F., and Lein, E.S. (2011). Cell-type-specific consequences of reelin deficiency in the mouse neocortex, hippocampus, and amygdala. J. Comp. Neurol. 519, 2061-2089.

Brecht, M. (2007). Barrel cortex and whisker-mediated behaviors. Curr. Opin. Neurobiol. 17, 408-416. van Brederode, J.F.M., Helliesen, M.K., and Hendrickson, A.E. (1991). Distribution of the calcium-binding proteins parvalbumin and calbindin-D28k in the sensorimotor cortex of the rat. Neuroscience 44, $157-171$.

Britanova, O., de Juan Romero, C., Cheung, A., Kwan, K.Y., Schwark, M., Gyorgy, A., Vogel, T., Akopov, S., Mitkovski, M., Agoston, D., et al. (2008). Satb2 Is a Postmitotic Determinant for Upper-Layer Neuron Specification in the Neocortex. Neuron 57, 378-392.

Brown, S.P., and Hestrin, S. (2009). Cell-type identity: a key to unlocking the function of neocortical circuits. Curr. Opin. Neurobiol. 19, 415-421.

Callaway, E. (2008). Transneuronal circuit tracing with neurotropic viruses. Curr. Opin. Neurobiol. 18, 617-623.

Callaway, E.M., and Luo, L. (2015). Monosynaptic Circuit Tracing with Glycoprotein-Deleted Rabies Viruses. J. Neurosci. 35, 8979-8985.

Caputi, A., Rozov, A., Blatow, M., and Monyer, H. (2009). Two calretinin-positive GABAergic cell types in layer $2 / 3$ of the mouse neocortex provide different forms of inhibition. Cereb. Cortex 19, 1345-1359.

Cardin, J.A., Carlén, M., Meletis, K., Knoblich, U., Zhang, F., Deisseroth, K., Tsai, L.-H., and Moore, C.I. (2009). Driving fast-spiking cells induces gamma rhythm and controls sensory responses. Nature $459,663-667$.

Caviness, V.S. (1976). Patterns of cell and fiber distribution in the neocortex of the reeler mutant mouse. J. Comp. Neurol. 170, 435-447.

Caviness, V.S., and Frost, D.O. (1983). Thalamocortical projections in the reeler mutant mouse. J. Comp. Neurol. 219, 182-202.

Caviness, V.S., and Rakic, P. (1978). Mechanisms of Cortical Development: A View From Mutations in Mice. Annu. Rev. Neurosci. 1, 297-326.

Caviness Jr., V.S., and Yorke Jr., C.H. (1976). Interhemispheric neocortical connections of the corpus callosum in the reeler mutant mouse: a study based on anterograde and retrograde methods. J. Comp. Neurol. 170, 449-460.

Celio, M.R. (1986). Parvalbumin in most y-aminobutyric acid-containing neurons of the rat cerebral cortex. Science (80-. ). 231, 995-997.

Chaudhary, R., and Rema, V. (2018). Deficits in Behavioral Functions of Intact Barrel Cortex Following Lesions of Homotopic Contralateral Cortex. Front. Syst. Neurosci. 12, 1-18.

Chen, N., Sugihara, H., and Sur, M. (2015). An acetylcholine-activated microcircuit drives temporal dynamics of cortical activity.

Chmielowska, J., Carvell, G.E., and Simons, D.J. (1989). Spatial organization of thalamocortical and corticothalamic projection systems in the rat Sml barrel cortex. J. Comp. Neurol. 285, 325-338.

Choi, J., and Callaway, E. (2011). Monosynaptic inputs to ErbB4-expressing inhibitory neurons in 
mouse primary somatosensory cortex. J. Comp. Neurol. 519, 3402-3414.

Cossell, L., lacaruso, M.F., Muir, D.R., Houlton, R., Sader, E.N., Ko, H., Hofer, S.B., and Mrsic-Flogel, T.D. (2015). Functional organization of excitatory synaptic strength in primary visual cortex. Nature 518, 399403.

Cruikshank, S., Urabe, H., Nurmikko, A., and Connors, B. (2010). Pathway-specific feedforward circuits between thalamus and neocortex revealed by selective optical stimulation of axons. Neuron 65 , 230-245.

Cruikshank, S.J., Ahmed, O.J., Stevens, T.R., Patrick, S.L., Gonzalez, A.N., Elmaleh, M., and Connors, B.W. (2012). Thalamic Control of Layer 1 Circuits in Prefrontal Cortex. J. Neurosci. 32, 17813-17823. D’Arcangelo, G., and Curran, T. (1998). Reeler: new tales on an old mutant mouse. Bioessays 20, 235-244.

DeFelipe, J. (2013). New insights into the classification and nomenclature of cortical GABAergic interneurons. Nat. Rev. Neurosci. 14, 202-216.

Dekimoto, H., Terashima, T., and Katsuyama, Y. (2010). Dispersion of the neurons expressing layer specific markers in the reeler brain. Dev. Growth Differ. 52, 181-193.

Delevich, K., Tucciarone, J., Huang, Z.J., and Li, B. (2015). The Mediodorsal Thalamus Drives Feedforward Inhibition in the Anterior Cingulate Cortex via Parvalbumin Interneurons. J. Neurosci. 35, 57435753.

DeNardo, L.A., Berns, D.S., DeLoach, K., and Luo, L. (2015). Connectivity of mouse somatosensory and prefrontal cortex examined with trans-synaptic tracing. Nat. Neurosci. 18, 1687-1697.

Denk, W., and Lichtman, J.W. (2011). The Big and the Small: Challenges of Imaging the Brain's Circuits. Science (80-. ). 334, 618-623.

Deshpande, A., Bergami, M., Ghanem, A., Conzelmann, K.K., Lepier, A., Gotz, M., and Berninger, B. (2013). Retrograde monosynaptic tracing reveals the temporal evolution of inputs onto new neurons in the adult dentate gyrus and olfactory bulb. Proc. Natl. Acad. Sci. U. S. A. 110, E1152-61.

Dietzschold, B., Li, J., Faber, M., and Schnell, M. (2008). Concepts in the pathogenesis of rabies. Futur. Viology 3, 481-490.

Diodato, A., Ruinart De Brimont, M., Yim, Y.S., Derian, N., Perrin, S., Pouch, J., Klatzmann, D., Garel, S., Choi, G.B., and Fleischmann, A. (2016). Molecular signatures of neural connectivity in the olfactory cortex. Nat. Commun. 7, 1-10.

Dipoppa, M., Ranson, A., Krumin, M., Pachitariu, M., Carandini, M., and Harris, K.D. (2018). Vision and locomotion shape the interactions between neuron types in mouse visual cortex. Neuron 98, 1-14. Do, J.P., Xu, M., Lee, S.-H., Chang, W.-C., Zhang, S., Chung, S., Yung, T.J., Fan, J.L., Miyamichi, K., Luo, L., et al. (2016). Cell type-specific long-range connections of basal forebrain circuit. Elife 5, 1-17. Donato, F., Rompani, S.B., and Caroni, P. (2013). Parvalbumin-expressing basket-cell network plasticity induced by experience regulates adult learning. Nature 504, 272-276.

Douglas, R.J., and Martin, K. a C. (2004). Neuronal circuits of the neocortex. Annu. Rev. Neurosci. 27, 419-451. 
Ebara, S., Kumamoto, K., Matsuura, T., Mazurkiewicz, J.E., and Rice, F.L. (2002). Similarities and differences in the innervation of mystacial vibrissal follicle-sinus complexes in the rat and cat: A confocal microscopic study. J. Comp. Neurol. 449, 103-119.

El-Boustani, S., and Sur, M. (2014). Response-dependent dynamics of cell-specific inhibition in cortical networks in vivo. Nat. Commun. 5, 1-14.

Fabri, M., and Burton, H. (1991). Ipsilateral cortical connections of primary somatic sensory cortex in rats. J. Comp. Neurol. 311, 405-424.

Faget, L., Osakada, F., Duan, J., Ressler, R., Johnson, A.B., Proudfoot, J.A., Yoo, J.H., Callaway, E.M., and Hnasko, T.S. (2016). Afferent Inputs to Neurotransmitter-Defined Cell Types in the Ventral Tegmental Area. Cell Rep. 15, 2796-2808.

Falconer, B.D.S. (1945). Two new mutants, "Trembler" and "Reeler", with neurological actions in the house mouse (Mus Musculus L.). J. Genet. 50, 192-205.

Fame, R.M., MacDonald, J.L., and Macklis, J.D. (2011). Development, specification, and diversity of callosal projection neurons. Trends Neurosci. 34, 41-50.

Favuzzi, E., Deogracias, R., Marques-Smith, A., Maeso, P., Jezequel, J., Exposito-Alonso, D., Balia, M., F. Maraver, E., Kroon, T., Hinojosa, A.J., et al. (2019). Distinct molecular programs regulate synapse specificity in cortical inhibitory circuits. Science (80-. ). 363, 413-417.

Feldmeyer, D., Brecht, M., Helmchen, F., Petersen, C.C.H., Poulet, J.F. a, Staiger, J.F., Luhmann, H.J., and Schwarz, C. (2013). Barrel cortex function. Prog. Neurobiol. 103, 3-27.

Feldmeyer, D., Qi, G., Emmenegger, V., and Staiger, J.F. (2017). Inhibitory interneurons and their circuit motifs in the many layers of the barrel cortex. Neuroscience.

Feldmeyer, D., Qi, G., Emmenegger, V., and Staiger, J.F. (2018). Inhibitory interneurons and their circuit motifs in the many layers of the barrel cortex. Neuroscience 368, 132-151.

Felleman, D.J., and Van Essen, D.C. (1991). Distributed hierachical processing in the primate cerebral cortex. Cereb. Cortex 1, 1-47.

Fenno, L., Mattis, J., Ramakrishnan, C., and Hyun, M. (2014). Targeting cells with single vectors using multiple-feature Boolean logic. Nat. Methods 11, 763-772.

Fenno, L.E., Mattis, J., Ramakrishnan, C., and Deisseroth, K. (2017). A guide to creating and testing new INTRSECT constructs. Curr. Protoc. Neurosci. 2017, 4.39.1-4.39.24.

Fino, E., and Yuste, R. (2011). Dense inhibitory connectivity in neocortex. Neuron 69, 1188-1203.

Freund, T.F., and Katona, I. (2007). Perisomatic Inhibition. Neuron 56, 33-42.

Fu, Y., Tucciarone, J.M., Espinosa, J.S., Sheng, N., Darcy, D.P., Nicoll, R. a, Huang, Z.J., and Stryker, M.P. (2014). A cortical circuit for gain control by behavioral state. Cell 156, 1139-1152.

Fu, Y., Kaneko, M., Tang, Y., Alvarez-Buylla, A., and Stryker, M.P. (2015). A cortical disinhibitory circuit for enhancing adult plasticity. Elife 2015, 1-12.

Fürth, D., Vaissière, T., Tzortzi, O., Xuan, Y., Märtin, A., Lazaridis, I., Spigolon, G., Fisone, G., Tomer, R., Deisseroth, K., et al. (2017). An interactive framework for whole-brain maps at cellular resolution. Nat. Neurosci. 21. 
Garcia-Junco-Clemente, P., Ikrar, T., Tring, E., Xu, X., Ringach, D.L., and Trachtenberg, J.T. (2017). An inhibitory pull-push circuit in frontal cortex. Nat. Neurosci. 20, 389-392.

Gentet, L.J., Kremer, Y., Taniguchi, H., Huang, Z.J., Staiger, J.F., and Petersen, C.C.H. (2012). Unique functional properties of somatostatin-expressing GABAergic neurons in mouse barrel cortex. Nat. Neurosci. 15, 607-612.

Ghanem, A., and Conzelmann, K.K. (2016). G gene-deficient single-round rabies viruses for neuronal circuit analysis. Virus Res. 216, 41-54.

Godwin, D.W., Che, D., O'Malley, D.M., and Zhou, Q. (1997). Photostimulation with caged neurotransmitters using fiber optic lightguides. J. Neurosci. Methods 73, 91-106.

Gonchar, Y., and Burkhalter, A. (1999). Connectivity of GABAergic Calretinin-immunoreactive Neurons in Rat Primary Visual Cortex. Cereb. Cortex 9, 683-696.

Goulas, A., Uylings, H.B.M., and Hilgetag, C.C. (2017). Principles of ipsilateral and contralateral cortico-cortical connectivity in the mouse. Brain Struct. Funct. 222, 1281-1295.

Greig, L.C., Woodworth, M.B., Galazo, M.J., Padmanabhan, H., and Macklis, J.D. (2013). Molecular logic of neocortical projection neuron specification, development and diversity. Nat. Rev. Neurosci. 14, 755-769.

Grinvald, A., Lieke, E., Frostig, R., Gilbert, C., and Wiesel, T. (1986). Functional architecture of cortex revealed by optical imaging of intrinsic signals. Nature $324,361-364$.

Guo, Q., Wang, D., He, X., Feng, Q., Lin, R., Xu, F., Fu, L., and Luo, M. (2015). Whole-Brain Mapping of Inputs to Projection Neurons and Cholinergic Interneurons in the Dorsal Striatum. PLoS One 10, e0123381.

Guy, J., and Staiger, J.F. (2017). The Functioning of a Cortex without Layers. Front. Neuroanat. 11, $1-13$.

Guy, J., Wagener, R.J., Möck, M., and Staiger, J.F. (2015). Persistence of Functional Sensory Maps in the Absence of Cortical Layers in the Somsatosensory Cortex of Reeler Mice. Cereb. Cortex 25, 2517-2528.

Guy, J., Sachkova, A., Möck, M., Witte, M., Wagener, R.J., and Staiger, J.F. (2016). Intracortical Network Effects Preserve Thalamocortical Input Efficacy in a Cortex Without Layers. Cereb. Cortex 1-16. Haider, B., Häusser, M., and Carandini, M. (2013). Inhibition dominates sensory responses in the awake cortex. Nature 493, 97-100.

Hamburgh, M. (1960). Observations on the neuropathology of "reeler", a neurological mutation in mice.

Experientia 16, 460-461.

Hamburgh, M. (1963). Analysis of the postnatal developmental effects of "reeler," a neurological mutation in mice. A study in developmental genetics. Dev. Biol. 8, 165-185.

Harris, K.D., and Shepherd, G.M.G. (2015). The neocortical circuit: themes and variations. Nat. Neurosci. 18, 170-181.

Harris, J.A., Mihalas, S., Hirokawa, K.E., Whitesell, J.D., Knox, J., Bernard, A., Bohn, P., Caldejon, S., Casal, L., Cho, A., et al. (2018). The organization of intracortical connections by layer and cell class in 
the mouse brain. BioRxiv 292961.

Harsan, L.-A., Dávid, C., Reisert, M., Schnell, S., Hennig, J., von Elverfeldt, D., and Staiger, J.F. (2013). Mapping remodeling of thalamocortical projections in the living reeler mouse brain by diffusion tractography. Proc. Natl. Acad. Sci. U. S. A. 110, E1797-806.

He, M., Tucciarone, J., Lee, S., Nigro, M.J., Kim, Y., Levine, J.M., Kelly, S.M., Krugikov, I., Wu, P., Chen, Y., et al. (2016). Strategies and tools for combinatorial targeting of GABAergic neurons in mouse cerebral cortex. Neuron 91, 1228-1243.

Helmstaedter, M., Briggman, K.L., Turaga, S.C., Jain, V., Seung, H.S., and Denk, W. (2013). Connectomic reconstruction of the inner plexiform layer in the mouse retina. Nature 500, 168-174.

Hevner, R.F., Daza, R.A.M., Englund, C., Kohtz, J., and Fink, A. (2004). Postnatal shifts of interneuron position in the neocortex of normal and reeler mice: Evidence for inward radial migration. Neuroscience 124, 605-618.

Hill, S.L., Wang, Y., Riachi, I., Schurmann, F., and Markram, H. (2012). Statistical connectivity provides a sufficient foundation for specific functional connectivity in neocortical neural microcircuits. Proc. Natl. Acad. Sci. 109, E2885-E2894.

Hioki, H., Okamoto, S., Konno, M., Kameda, H., Sohn, J., Kuramoto, E., Fujiyama, F., and Kaneko, T. (2013). Cell Type-Specific Inhibitory Inputs to Dendritic and Somatic Compartments of Parvalbumin-Expressing Neocortical Interneuron. J. Neurosci. 33, 544-555.

Hippenmeyer, S., Vrieseling, E., Sigrist, M., Portmann, T., Laengle, C., Ladle, D.R., and Arber, S. (2005). A developmental switch in the response of DRG neurons to ETS transcription factor signaling. PLoS Biol. 3, 0878-0890.

Hu, H., Ma, Y., and Agmon, A. (2011). Submillisecond Firing Synchrony between Different Subtypes of Cortical Interneurons Connected Chemically But Not Electrically. J. Neurosci. 31, 3351-3361.

Hu, H., Gan, J., and Jonas, P. (2014). Interneurons. Fast-spiking, parvalbumin+ GABAergic interneurons: from cellular design to microcircuit function. Science (80-. ). 345, 1255263-1-1255263-13.

Imai, H., Yamamoto, T., Katsuyama, Y., Kikkawa, S., and Terashima, T. (2012). Subcortically and callosally projecting neurons are distinct neuronal pools in the motor cortex of the Reeler mouse. Kobe J. Med. Sci. 58, 86-95.

Innocenti, G. (1981). Growth and reshaping of axons in the establishment of visual callosal connections. Science (80-. ). 212, 824-826.

Innocenti, G.M., and Price, D.J. (2005). Exuberance in the development of cortical networks. Nat. Rev. Neurosci. 6, 955-965.

Isaacson, J., and Scanziani, M. (2011). How inhibition shapes cortical activity. Neuron 72, 231-243.

Ivy, G.O., Could, H.J., and Killackey, H.P. (1984). Variability in the distribution of callosal projection neurons in the adult rat parietal cortex. Brain Res. 306, 53-61.

Jackson, J., Ayzenshtat, I., Karnani, M.M., and Yuste, R. (2016). VIP+ interneurons control neocortical activity across brain states. J. Neurophysiol. 115, 3008-3017.

Ji, X. -y., Zingg, B., Mesik, L., Xiao, Z., Zhang, L.I., and Tao, H.W. (2015). Thalamocortical Innervation 
Pattern in Mouse Auditory and Visual Cortex: Laminar and Cell-Type Specificity. Cereb. Cortex 1-14. Jiang, X., Shen, S., Cadwell, C.R., Berens, P., Sinz, F., Ecker, A.S., Patel, S., and Tolias, A.S. (2015). Principles of connectivity among morphologically defined cell types in adult neocortex. Science (80-. ). 350, aac9462-1-aac9462-10.

Kapfer, C., Glickfeld, L.L., Atallah, B. V., and Scanziani, M. (2007). Supralinear increase of recurrent inhibition during sparse activity in the somatosensory cortex. Nat. Neurosci. 10, 743-753.

Karnani, M.M., Agetsuma, M., and Yuste, R. (2014). A blanket of inhibition: Functional inferences from dense inhibitory connectivity. Curr. Opin. Neurobiol. 26, 96-102.

Karnani, M.M., Jackson, J., Ayzenshtat, I., Tucciarone, J., Manoocheri, K., Snider, W.G., and Yuste, R. (2016a). Cooperative Subnetworks of Molecularly Similar Interneurons in Mouse Neocortex. Neuron $1-15$.

Karnani, X.M.M., Jackson, J., Ayzenshtat, I., Sichani, X.A.H., Manoocheri, K., Kim, S., and Yuste, R. (2016b). Opening Holes in the Blanket of Inhibition : Localized Lateral Disinhibition by VIP Interneurons. J. Neurosci. 36, 3471-3480.

Kasthuri, N., Hayworth, K.J., Berger, D.R., Schalek, R.L., Conchello, J.A., Knowles-Barley, S., Lee, D., Vázquez-Reina, A., Kaynig, V., Jones, T.R., et al. (2015). Saturated Reconstruction of a Volume of Neocortex. Cell 162, 648-661.

Kawaguchi, Y., and Kubota, Y. (1997). GABAergic cell subtypes and their synaptic connections in rat frontal cortex. Cereb. Cortex 7, 476-486.

Kelly, R.M., and Strick, P.L. (2000). Rabies as a transneuronal tracer of circuits in the central nervous system. J. Neurosci. Methods 103, 63-71.

Kim, E.J., Juavinett, A.L., Kyubwa, E.M., Jacobs, M.W., and Callaway, E.M. (2015). Three Types of Cortical Layer 5 Neurons That Differ in Brain-wide Connectivity and Function. Neuron 88.

Kim, E.J., Jacobs, M.W., Ito-cole, T., and Callaway, E.M. (2016). Improved Monosynaptic Neural Circuit Tracing Using Engineered Rabies Virus Glycoproteins Report. CellReports 15, 1-8.

Kim, M.-H., Znamenskiy, P., lacaruso, M.F., and Mrsic-Flogel, T.D. (2018). Segregated Subnetworks of Intracortical Projection Neurons in Primary Visual Cortex. Neuron 100, 1313-1321.e6.

Koelbl, C., Helmstaedter, M., Luebke, J., and Feldmeyer, D. (2015). A barrel-related interneuron in layer 4 of rat somatosensory cortex with a high intrabarrel connectivity. Cereb. Cortex 25, 713-725.

Kubo, K., and Nakajima, K. (2003). Cell and molecular mechanisms that control cortical layer formation in the brain. Keio J.Med. 52, 8-20.

Kuchibhotla, K. V, Gill, J. V, Lindsay, G.W., Papadoyannis, E.S., Field, R.E., Sten, T.A.H., Miller, K.D., and Froemke, R.C. (2016). Parallel processing by cortical inhibition enables context-dependent behavior. Nat. Neurosci. 1-14.

Kuhlman, S.J., Olivas, N.D., Tring, E., Ikrar, T., Xu, X., and Trachtenberg, J.T. (2013). A disinhibitory microcircuit initiates critical-period plasticity in the visual cortex. Nature 501, 543-546.

Kuypers, H.G., and Ugolini, G. (1990). Viruses as transneuronal tracers. Trends Neurosci. 13, 71-75.

Larsen, D.D., Wickersham, I.R., and Callaway, E.M. (2007). Retrograde tracing with recombinant ra- 
bies virus reveals correlations between projection targets and dendritic architecture in layer 5 of mouse barrel cortex. Front. Neural Circuits 1, 5.

Lee, G.H., and D’Arcangelo, G. (2016). New Insights into Reelin-Mediated Signaling Pathways. Front. Cell. Neurosci. 10, 1-8.

Lee, A.J., Wang, G., Jiang, X., Johnson, S.M., Hoang, E.T., Lanté, F., Stornetta, R.L., Beenhakker, M.P., Shen, Y., and Julius Zhu, J. (2015). Canonical Organization of Layer 1 Neuron-Led Cortical Inhibitory and Disinhibitory Interneuronal Circuits. Cereb. Cortex 25, 2114-2126.

Lee, S.-H., Kwan, A.C., Zhang, S., Phoumthipphavong, V., Flannery, J.G., Masmanidis, S.C., Taniguchi, H., Huang, Z.J., Zhang, F., Boyden, E.S., et al. (2012). Activation of specific interneurons improves V1 feature selectivity and visual perception. Nature 488, 379-383.

Lee, S., Hjerling-Leffler, J., Zagha, E., Fishell, G., and Rudy, B. (2010). The largest group of superficial neocortical GABAergic interneurons expresses ionotropic serotonin receptors. J. Neurosci. 30, 16796-16808.

Lee, S., Kruglikov, I., Huang, Z.J., Fishell, G., and Rudy, B. (2013). A disinhibitory circuit mediates motor integration in the somatosensory cortex. Nat. Neurosci. 16, 1662-1670.

Lee, S.B., Lee, H.S., and Waterhouse, B.D. (2008). The collateral projection from the dorsal raphe nucleus to whisker-related, trigeminal sensory and facial motor systems in the rat. Brain Res. 1214, $11-22$.

Lefort, S., Tomm, C., Floyd Sarria, J.C., and Petersen, C.C.H. (2009). The Excitatory Neuronal Network of the C2 Barrel Column in Mouse Primary Somatosensory Cortex. Neuron 61, 301-316.

Lemus, L., Ndez, A.H., Luna, R., Zainos, A., and Romo, R. (2010). Do sensory cortices process more than one sensory modality during perceptual judgments. Neuron 67, 335-348.

Letzkus, J.J., Wolff, S.B.E., Meyer, E.M.M., Tovote, P., Courtin, J., Herry, C., and Lüthi, A. (2011). A disinhibitory microcircuit for associative fear learning in the auditory cortex. Nature 480, 331-335.

Leyva-Díaz, E., and López-Bendito, G. (2013). In and out from the cortex: Development of major forebrain connections. Neuroscience 254, 26-44.

Lichtman, J.W., and Sanes, J.R. (2008). Ome sweet ome: what can the genome tell us about the connectome. Curr. Opin. Neurobiol. 18, 346-353.

Lin, S., Brown, R.E., Shuler, M.G.H., and Petersen, C.C.H. (2015). Optogenetic dissection of the basal forebrain neuromodulatory control of cortical activation, plasticity and cognition. J. Neurosci. 35, 1-20.

Loewy, A.D. (1998). Viruses as transneuronal tracers for defining neural circuits. Neurosci. Biobehav. Rev. 22, 679-684.

Luo, L., Callaway, E.M., and Svoboda, K. (2008). Genetic dissection of neural circuits. Neuron 57, 634-660.

Ma, P.M., and Woolsey, T. a (1984). Cytoarchitectonic correlates of the vibrissae in the medullary trigeminal complex of the mouse. Brain Res. 306, 374-379.

Ma, Y., Hu, H., Berrebi, A.S., Mathers, P.H., and Agmon, A. (2006). Distinct subtypes of somatostatin-containing neocortical interneurons revealed in transgenic mice. J. Neurosci. 26, 5069-5082. 
Ma, Y., Hu, H., and Agmon, A. (2013). Short-Term Plasticity of Unitary Inhibitory-to-Inhibitory Synapses Depends on the Presynaptic Interneuron Subtype. J. Neurosci. 322, 983-988.

Madisen, L., Garner, A.R., Shimaoka, D., Chuong, A.S., Klapoetke, N.C., Li, L., van der Bourg, A., Niino, Y., Egolf, L., Monetti, C., et al. (2015). Transgenic Mice for Intersectional Targeting of Neural Sensors and Effectors with High Specificity and Performance. Neuron 85, 942-958.

Mao, T., Kusefoglu, D., Hooks, B.M., Huber, D., Petreanu, L., and Svoboda, K. (2011). Long-Range Neuronal Circuits Underlying the Interaction between Sensory and Motor Cortex. Neuron 72, 111-123.

De Marco García, N. V, Priya, R., Tuncdemir, S.N., Fishell, G., and Karayannis, T. (2015). Sensory inputs control the integration of neurogliaform interneurons into cortical circuits. Nat. Neurosci. 18, 393-401.

Markram, H., Toledo-Rodriguez, M., Wang, Y., Gupta, A., Silberberg, G., and Wu, C. (2004). Interneurons of the neocortical inhibitory system. Nat. Rev. Neurosci. 5, 793-807.

Marshel, J.H., Mori, T., Nielsen, K.J., and Callaway, E.M. (2010). Targeting Single Neuronal Networks for Gene Expression and Cell Labeling In Vivo. Neuron 67, 562-574.

Maruyama, A.T., and Komai, S. (2018). Auditory-induced response in the primary sensory cortex of rodents. PLoS One 13, e0209266.

Massé, I.O., Ross, S., Bronchti, G., and Boire, D. (2016). Asymmetric Direct Reciprocal Connections Between Primary Visual and Somatosensory Cortices of the Mouse. Cereb. Cortex 1-18.

Mebatsion, T., Konig, M., and Conzelmann, K.K. (1996). Budding of rabies virus particles in the absence of the spike glycoprotein. Cell 84, 941-951.

Meyer, H.S., Schwarz, D., Wimmer, V.C., Schmitt, A.C., Kerr, J.N.D., Sakmann, B., and Helmstaedter, M. (2011). Inhibitory interneurons in a cortical column form hot zones of inhibition in layers 2 and $5 A$. Proc. Natl. Acad. Sci. 108, 16807-16812.

Meyer, H.S., Egger, R., Guest, J.M., Foerster, R., Reissl, S., and Oberlaender, M. (2013). Cellular organization of cortical barrel columns is whisker-specific. Proc. Natl. Acad. Sci. U. S. A. 110, 1911319118.

Mijamichi, K., Yael, S.-F., Marvin, S., Brandon C., W., Liqun, L., and Mizrahi, A. (2013). Dissecting local circuits: parvalbumin interneurons underlie broad feedback control of olfactory bulb output. Neuron 80 , 1232-1245.

Miller, M.W., and Vogt, B. a (1984). Direct connections of rat visual cortex with sensory, motor, and association cortices. J. Comp. Neurol. 226, 184-202.

Minskaia, E., Nicholson, J., and Ryan, M.D. (2013). Optimisation of the foot-and-mouth disease virus 2A co-expression system for biomedical applications. BMC Biotechnol. 13, 67.

Molyneaux, B.J., Arlotta, P., Fame, R.M., MacDonald, J.L., MacQuarrie, K.L., and Macklis, J.D. (2009). Novel Subtype-Specific Genes Identify Distinct Subpopulations of Callosal Projection Neurons. J. Neurosci. 29, 12343-12354.

Muñoz, W., Tremblay, R., Levenstein, D., and Rudy, B. (2017). Layer-specific modulation of neocortical dendritic inhibition during active wakefulness. Science (80-. ). 355, 954-959. 
Narayanan, R.T., Egger, R., Johnson, A.S., Mansvelder, H.D., Sakmann, B., De Kock, C.P.J., and Oberlaender, M. (2015). Beyond columnar organization: Cell type- and target layer-specific principles of horizontal axon projection patterns in rat vibrissal cortex. Cereb. Cortex 25, 4450-4468.

Nassi, J.J., Cepko, C.L., Born, R.T., and Beier, K.T. (2015). Neuroanatomy goes viral! Front. Neuroanat. 9, 1-24.

Nelson, A., and Mooney, R. (2016). The Basal Forebrain and Motor Cortex Provide Convergent yet Distinct Movement-Related Inputs to the Auditory Cortex. Neuron 90, 1-14.

Nigro, M.J., Hashikawa, Y., and Rudy, B. (2018). Diversity and connectivity of layer 5 somatostatin-expressing interneurons in the mouse barrel cortex. J. Neurosci. 38, 2415-2417.

O'Leary, D.D.M. (1992). Development of connectional diversity and specificity in the mammalian brain by the pruning of collateral projections. Curr. Opin. Neurobiol. 2, 70-77.

O'Leary, D.D.M., Stanfield, B.B., and Cowan, W.M. (1981). Evidence that the early postnatal restriction of the cells of origin of the callosal projection is due to the elimination of axonal collaterals rather than to the death of neurons. Dev. Brain Res. 1, 607-617.

Osakada, F., Mori, T., Cetin, A.H., Marshel, J.H., Virgen, B., and Callaway, E.M. (2011). New rabies virus variants for monitoring and manipulating activity and gene expression in defined neural circuits. Neuron 71, 617-631.

Oswald, M., Geissler, S., and Goepferich, A. (2017). Targeting the central nervous system ( CNS ): a review of rabies virus - targeting strategies. Mol. Pharm. 14, 2177-2196.

Packer, A.M., and Yuste, R. (2011). Dense, Unspecific Connectivity of Neocortical Parvalbumin-Positive Interneurons: A Canonical Microcircuit for Inhibition? J. Neurosci. 31, 13260-13271.

Pakan, J.M., Lowe, S.C., Dylda, E., Keemink, S.W., Currie, S.P., Coutts, C.A., and Rochefort, N.L. (2016). Behavioral-state modulation of inhibition is context-dependent and cell type specific in mouse visual cortex. Elife 5, 7250-7257.

Paxinos, G. and Franklin, K.B.J. (2001) The Mouse Brain in Stereotaxic Coordinates. 2nd Edition, Academic Press, San Diego.

Perin, R., Berger, T.K., and Markram, H. (2011). A synaptic organizing principle for cortical neuronal groups. Proc. Natl. Acad. Sci. U. S. A. 108, 5419-5424.

Petersen, C.C.H. (2007). The functional organization of the barrel cortex. Neuron 56, 339-355.

Petreanu, L., Huber, D., Sobczyk, A., and Svoboda, K. (2007). Channelrhodopsin-2-assisted circuit mapping of long-range callosal projections. Nat. Neurosci. 10, 663-668.

Pfeffer, C.K. (2014). Inhibitory neurons: vip cells hit the brake on inhibition. Curr. Biol. 24, 18-20.

Pfeffer, C.K., Xue, M., He, M., Huang, Z.J., and Scanziani, M. (2013). Inhibition of inhibition in visual cortex: the logic of connections between molecularly distinct interneurons. Nat. Neurosci. 16, 10681076.

Pi, H., Hangya, B., Kvitsiani, D., and Sanders, J. (2013). Cortical interneurons that specialize in disinhibitory control. Nature 503, 521-524.

Pielecka-Fortuna, J., Wagener, R.J., Martens, A.K., Goetze, B., Schmidt, K.F., Staiger, J.F., and Löwel, 
S. (2014). The disorganized visual cortex in reelin-deficient mice is functional and allows for enhanced plasticity. Brain Struct. Funct.

Polleux, F., Dehay, C., and Kennedy, H. (1998). Neurogenesis and Commitment of Corticospinal Neurons in reeler. J. Neurosci. 18, 9910-9923.

Pouille, F., and Scanziani, M. (2001). Enforcement of temporal fidelity in pyramidal cells by feed-forward somatic inhibition. Science (80-. ). 293, 325-331.

Pouille, F., Marin-Burgin, A., Adesnik, H., Atallah, B. V., and Scanziani, M. (2009). Input normalization by global feedforward inhibition expands cortical dynamic range. Nat. Neurosci. 12, 1577-1585.

Poulin, J., Tasic, B., Hjerling-Leffler, J., Trimarchi, J.M., and Awatramani, R. (2016). Disentangling neural cell diversity using single-cell transcriptomics. Nat. Neurosci. 19, 1131-1141.

Price, D.J., and Blakemore, C. (1985). Regressive events in the postnatal development of association projections in the visual cortex. Nature 316, 452-457.

Prönneke, A., Scheuer, B., Wagener, R.J., Möck, M., Witte, M., and Staiger, J.F. (2015). Characterizing VIP Neurons in the Barrel Cortex of VIPcre/tdTomato Mice Reveals Layer-Specific Differences. Cereb. Cortex 25, 4854-4868.

Rajasethupathy, P., Sankaran, S., Marshel, J.H., Kim, C.K., Ferenczi, E., Lee, S.Y., Berndt, A., Ramakrishnan, C., Jaffe, A., Lo, M., et al. (2015). Projections from neocortex mediate top-down control of memory retrieval. Nature 526, 653-659.

Rancz, E.A., Franks, K.M., Schwarz, M.K., Pichler, B., Schaefer, A.T., and Margrie, T.W. (2011). Transfection via whole-cell recording in vivo : bridging single- cell physiology, genetics and connectomics. Nat. Neurosci. 14, 527-532.

Rong, L., Gendron, K., Strohl, B., Shenoy, R., Wool-lewis, R.J., and Bates, P. (1998). Characterization of Determinants for Envelope Binding and Infection in Tva , the Subgroup A Avian Sarcoma and Leukosis Virus Receptor. 72, 4552-4559.

Roux, L., and Buzsáki, G. (2015). Tasks for inhibitory interneurons in intact brain circuits. Neuropharmacology 88, 10-23.

Rudy, B., Fishell, G., Lee, S., and Hjerling-Leffler, J. (2011). Three groups of interneurons account for nearly $100 \%$ of neocortical GABAergic neurons. Dev. Neurobiol. 71, 45-61.

Sachidhanandam, S., Sermet, B.S., and Petersen, C.C.H. (2016). Parvalbumin-Expressing GABAergic Neurons in Mouse Barrel Cortex Contribute to Gating a Goal-Directed Sensorimotor Transformation. Cell Rep. 15, 700-706.

Salinger, W.L., Ladrow, P., and Wheeler, C. (2003). Behavioral Phenotype of the Reeler Mutant Mouse: Effects of Reln Gene Dosage and Social Isolation. Behav. Neurosci. 117, 1257-1275.

Schindelin, J., Arganda-Carreras, I., Frise, E., Kaynig, V., Longair, M., Pietzsch, T., Preibisch, S., Rueden, C., Saalfeld, S., Schmid, B., et al. (2012). Fiji: An open-source platform for biological-image analysis. Nat. Methods 9, 676-682.

Schubert, D., Kötter, R., and Staiger, J.F. (2007). Mapping functional connectivity in barrel-related columns reveals layer- and cell type-specific microcircuits. Brain Struct. Funct. 212, 107-119. 
Schuman, B., Machold, R., Hashikawa, Y., Fuzik, J., Fishell, G., and Rudy, B. (2018). Four unique interneuron populations reside in neocortical layer 1. J. Neurosci. 39, 1613-1618.

Schwarz, L. a., Miyamichi, K., Gao, X.J., Beier, K.T., Weissbourd, B., DeLoach, K.E., Ren, J., Ibanes, S., Malenka, R.C., Kremer, E.J., et al. (2015). Viral-genetic tracing of the input-output organization of a central noradrenaline circuit. Nature 524, 88-92.

Sieben, K., Roder, B., and Hanganu-Opatz, I.L. (2013). Oscillatory Entrainment of Primary Somatosensory Cortex Encodes Visual Control of Tactile Processing. J. Neurosci. 33, 5736-5749.

Silberberg, G., and Markram, H. (2007). Disynaptic Inhibition between Neocortical Pyramidal Cells Mediated by Martinotti Cells. Neuron 53, 735-746.

Silberberg, G., Gupta, A., and Markram, H. (2002). Stereotypy in neocortical microcircuits. Trends Neurosci. 25, 227-230.

Simons, D.J., and Woolsey, T. a. (1979). Functional organization in mouse barrel cortex. Brain Res. 165, 327-332.

Sohal, V.S., Zhang, F., Yizhar, O., and Deisseroth, K. (2009). Parvalbumin neurons and gamma rhythms enhance cortical circuit performance. Nature 459, 698-702.

Sohn, J., Okamoto, S., Kataoka, N., and Kaneko, T. (2016). Differential Inputs to the Perisomatic and Distal-Dendritic Compartments of VIP-Positive Neurons in Layer 2 / 3 of the Mouse Barrel Cortex. 10, $1-18$.

Somogyi, P. (1977). A specific "axo-axonal" interneuron in the visual cortex of the rat. Brain Res. 136, 345-350.

Song, S., Sjöström, P.J., Reigl, M., Nelson, S., and Chklovskii, D.B. (2005). Highly nonrandom features of synaptic connectivity in local cortical circuits. PLoS Biol. 3, 0507-0519.

Sparta, D.R., Hovelso, N., Mason, A.O., Kantak, P.A., Ung, R.L., Decot, H.K., and Stuber, G.D. (2014). Activation of Prefrontal Cortical Parvalbumin Interneurons Facilitates Extinction of Reward-Seeking Behavior. J. Neurosci. 34, 3699-3705.

Staiger, J.F., Zilles, K., and Freund, T.F. (1996a). Distribution of GABAergic elements postsynaptic to ventroposteromedial thalamic projections in layer IV of rat barrel cortex. Eur. J. Neurosci. 8, 2273-2285.

Staiger, J.F., Zilles, K., and Freund, T.F. (1996b). Innervation of VIP-immunoreactive neurons by the ventroposteromedial thalamic nucleus in the barrel cortex of the rat. J. Comp. Neurol. 367, 194-204.

Staiger, J.F., Masanneck, C., Schleicher, A., and Zuschratter, W. (2004). Calbindin-containing interneurons are a target for VIP-immunoreactive synapses in rat primary somatosensory cortex. J. Comp. Neurol. 468, 179-189.

Staiger, J.F., Zuschratter, W., Luhmann, H.J., and Schubert, D. (2009). Local circuits targeting parvalbumin-containing interneurons in layer IV of rat barrel cortex. Brain Struct. Funct. 214, 1-13.

Staiger, J.F., Bojak, I., Miceli, S., and Schubert, D. (2015). A gradual depth-dependent change in connectivity features of supragranular pyramidal cells in rat barrel cortex. Brain Struct. Funct. 220, 13171337.

Stanfield, B.B., and Cowan, W.M. (1979). The development of the hippocampus and dentate gyrus in 
normal and reeler mice. J. Comp. Neurol. 185, 423-459.

Steindler, D.A., and Colwell, S.A. (1976). Reeler mutant mouse: maintenance of appropriate and reciprocal connections in the cerebral cortex and thalamus. Brain Res. 105, 386-393.

Tamás, G., Somogyi, P., and Buhl, E.H. (1998). Differentially interconnected networks of GABAergic interneurons in the visual cortex of the cat. J. Neurosci. 18, 4255-4270.

Taniguchi, H., He, M., Wu, P., Kim, S., Paik, R., Sugino, K., Kvitsiani, D., Kvitsani, D., Fu, Y., Lu, J., et al. (2011). A resource of Cre driver lines for genetic targeting of GABAergic neurons in cerebral cortex. Neuron 71, 995-1013.

Tasic, B., Menon, V., Nguyen, T.N., Kim, T.K., Jarsky, T., Yao, Z., Levi, B., Gray, L.T., Sorensen, S.A., Dolbeare, T., et al. (2016). Adult mouse cortical cell taxonomy revealed by single cell transcriptomics. Nat. Neurosci. 19, 335-346.

Tasic, B., Yao, Z., Smith, K.A., Graybuck, L., Nguyen, T.N., Bertagnolli, D., Goldy, J., Garren, E., Economo, M.N., Viswanathan, S., et al. (2018). Shared and distinct transcriptomic cell types across neocortical areas. Nature 563, 229542.

Tervo, D.G.R., Hwang, B.Y., Viswanathan, S., Gaj, T., Lavzin, M., Ritola, K.D., Lindo, S., Michael, S., Kuleshova, E., Ojala, D., et al. (2016). A Designer AAV Variant Permits Efficient Retrograde Access to Projection Neurons. Neuron 92, 372-382.

Tremblay, R., Lee, S., and Rudy, B. (2016). GABAergic Interneurons in the Neocortex: From Cellular Properties to Circuits. Neuron 91, 260-292.

Ugolini, G. (1995). Specificity of rabies virus as a transneuronal tracer of motor networks: Transfer from hypoglossal motoneurons to connected second-order and higher order central nervous system cell groups. J. Comp. Neurol. 356, 457-480.

Ugolini, G. (2010). Advances in viral transneuronal tracing. J. Neurosci. Methods 194, 2-20.

Urban-Ciecko, J., and Barth, A.L. (2016). Somatostatin-expressing neurons in cortical networks. Nat. Rev. Neurosci. 17, 401-409.

Velez-Fort, M., Rousseau, C. V, Niedworok, C.J., Wickersham, I.R., Rancz, E. a, Brown, A.P.Y., Strom, M., and Margrie, T.W. (2014). The Stimulus Selectivity and Connectivity of Layer Six Principal Cells Reveals Cortical Microcircuits Underlying Visual Processing. Neuron 83, 1431-1443.

Vucurovic, K., Gallopin, T., Ferezou, I., Rancillac, A., Chameau, P., van Hooft, J. a, Geoffroy, H., Monyer, H., Rossier, J., and Vitalis, T. (2010). Serotonin 3A receptor subtype as an early and protracted marker of cortical interneuron subpopulations. Cereb. Cortex 20, 2333-2347.

Wagener, R.J., Dávid, C., Zhao, S., Haas, C. a, and Staiger, J.F. (2010). The somatosensory cortex of reeler mutant mice shows absent layering but intact formation and behavioral activation of columnar somatotopic maps. J. Neurosci. 30, 15700-15709.

Wagener, R.J., Witte, M., Guy, J., Mingo-Moreno, N., Ku gler, S., and Staiger, J.F. (2015). Thalamocortical Connections Drive Intracortical Activation of Functional Columns in the Mislaminated Reeler Somatosensory Cortex. Cereb. Cortex 1-18.

Walker, F., Feyerabend, M., Guy, J., Wagener, R.J., Schubert, D., Staiger, J.F., Witte, M., Feyerabend, M., Guy, J., Staiger, J.F., et al. (2016). Parvalbumin- and Vasoactive intestinal polypeptide-expressing 
neocortical interneurons impose differential inhibition on Martinotti cells.

Wall, N.R., Wickersham, I.R., Cetin, A., De La Parra, M., and Callaway, E.M. (2010). Monosynaptic circuit tracing in vivo through Cre-dependent targeting and complementation of modified rabies virus. Proc. Natl. Acad. Sci. U. S. A. 107, 21848-21853.

Wall, N.R., Parra, M.D. La, Sorokin, J.M., Taniguchi, H., Huang, Z.J., and Callaway, E.M. (2016). BrainWide Maps of Synaptic Input to Cortical Interneurons. J. Neurosci. 36, 4000-4009.

Wang, Y., Gupta, A., Toledo-Rodriguez, M., Wu, C.Z., and Markram, H. (2002). Anatomical , Physiological , Molecular and Circuit Properties of Nest Basket Cells in the Developing Somatosensory Cortex. Cereb. Cortex 12, 395-410.

Wang, Y., Toledo-Rodriguez, M., Gupta, A., Wu, C., Silberberg, G., Luo, J., and Markram, H. (2004). Anatomical, physiological and molecular properties of Martinotti cells in the somatosensory cortex of the juvenile rat. J. Physiol. 561, 65-90.

Watabe-Uchida, M., Zhu, L., Ogawa, S.K., Vamanrao, A., and Uchida, N. (2012). Whole-Brain Mapping of Direct Inputs to Midbrain Dopamine Neurons. Neuron 74, 858-873.

Weible, A.P., Schwarcz, L., Wickersham, I.R., Deblander, L., Wu, H., Callaway, E.M., Seung, H.S., and Kentros, C.G. (2010). Transgenic targeting of recombinant rabies virus reveals monosynaptic connectivity of specific neurons. J. Neurosci. 30, 16509-16513.

Weissbourd, B., Ren, J., DeLoach, K.E., Guenthner, C.J., Miyamichi, K., and Luo, L. (2014). Presynaptic Partners of Dorsal Raphe Serotonergic and GABAergic Neurons. Neuron 83, 645-662.

Wertz, A., Trenholm, S., Yonehara, K., Hillier, D., Raics, Z., Leinweber, M., Szalay, G., Ghanem, A., Keller, G., Rózsa, B., et al. (2015). Single-cell-initiated monosynaptic tracing reveals layer-specific cortical network modules. Science (80-. ). 349, 70-74.

White, E.L., and DeAmicis, R.A. (1977). Afferent and efferent projections of the region in mouse SmL cortex which contains the posteromedial barrel subfield. J. Comp. Neurol. 175, 455-482.

Wickersham, I.R., and Feinberg, E.H. (2012). New technologies for imaging synaptic partners. Curr. Opin. Neurobiol. 22, 121-127.

Wickersham, I.R., Lyon, D.C., Barnard, R.J.O., Mori, T., Conzelmann, K., Young, J.A.T., and Callaway, E.M. (2007a). Monosynaptic Restriction of Transsynaptic Tracing from Single, Genetically Targeted Neurons. Neuron 53, 639-647.

Wickersham, I.R., Finke, S., Conzelmann, K.-K., and Callaway, E.M. (2007b). Retrograde neuronal tracing with a deletion-mutant rabies virus. Nat. Methods 4, 47-49.

Williams, L.E., and Holtmaat, A. (2019). Higher-order thalamocortical inputs gate synaptic long-term potentiation via disinhibiton. Neuron 101, 1-12.

Wilson, N.R., Runyan, C.A., Wang, F.L., and Sur, M. (2012). Division and subtraction by distinct cortical inhibitory networks in vivo. Nature 488, 343-348.

Wise, S.P., and Jones, E.G. (1976). The organization and postnatal development of the commissural projection of the rat somatic sensory cortex. J. Comp. Neurol. 168, 313-343.

Woolsey, T., and Loos, H. Van der (1970). The structural organization of layer IV in the somatosensory 
region (SI) of mouse cerebral cortex: the description of a cortical field composed of discrete cytoarchitectonic units. Brain Res. 17, 205-242.

Xu, H., Jeong, H.Y., Tremblay, R., and Rudy, B. (2013). Neocortical Somatostatin-Expressing GABAergic Interneurons Disinhibit the Thalamorecipient Layer 4. Neuron 77, 155-167.

Xu, X., Roby, K.D., and Callaway, E.M. (2006). Mouse cortical inhibitory neuron type that coexpresses somatostatin and calretinin. J. Comp. Neurol. 499, 144-160.

Yabut, O., Renfro, A., Niu, S., Swann, J.W., Marín, O., and D’Arcangelo, G. (2007). Abnormal laminar position and dendrite development of interneurons in the reeler forebrain. Brain Res. 1140, 75-83.

Yavorska, I., and Wehr, M. (2016). Somatostatin-Expressing Inhibitory Interneurons in Cortical Circuits. Front. Neural Circuits 10, 1-18.

Yazaki-Sugiyama, Y., Kang, S., Cteau, H., Fukai, T., and Hensch, T.K. (2009). Bidirectional plasticity in fast-spiking GABA circuits by visual experience. Nature 462, 218-221.

Zeng, H., and Sanes, J.R. (2017). Neuronal cell-type classification: challenges, opportunities and the path forward. Nat. Rev. Neurosci. 18, 530-546.

Zhang, L.I., and Poo, M.M. (2001). Electrical activity of neural circuits. Nat. Neurosci. 4, 1207-1214.

Zhang, S., Xu, M., Kamigaki, T., Hoang Do, J.P., Chang, W.-C., Jenvay, S., Miyamichi, K., Luo, L., and Dan, Y. (2014). Long-range and local circuits for top-down modulation of visual cortex processing. Science (80-. ). 345, 660-665.

Zhang, S., Xu, M., Chang, W.-C., Ma, C., Hoang Do, J.P., Jeong, D., Lei, T., Fan, J.L., and Dan, Y. (2016). Organization of long-range inputs and outputs of frontal cortex for top-down control. Nat. Neurosci. 1-12.

Zhou, X., Rickmann, M., Hafner, G., and Staiger, J.F. (2017). Subcellular Targeting of VIP Boutons in Mouse Barrel Cortex is Layer-Dependent and not Restricted to Interneurons. Cereb. Cortex 24, 1-16. 


\section{STATEMENT OF AUTHORSHIP}

This work has emerged from a team effort that involved many people across the globe. Here I would like to explicitly state what kind of work in this thesis has been contributed by others. Jochen Staiger, Mirko Witte, Julien Guy were the main people who partook in the design of the experiments and who I discussed the results with. Lief Fenno, Charu Ramakrishnan, Yoon S. Kim and Karl Deisseroth engineered the intersectional helper viruses for RV-tracing and kindly shared them with us. Michael Lingelbach and Edward Callaway engineered the Cre-dependent helper virus for RV-tracing that was finally used for our experiments. Ian Wickersham, Martin Schwarz and Karl Deisseroth donated Cre-dependent helper viruses that we tested and compared in pursuit to find an ideal construct for our question. Karl-Klaus Conzelmann kindly donated the RVs.

Nidhi Subhashini performed the glutamate uncaging experiments and Martin Möck wrote the scripts for analysis of these data. Julien Guy constructed the intrinsic signal optical imaging setup. Mouse lines were breed and maintained by Mirko Witte and Julien Guy. Patricia Sprysch and Sandra Heinzl provided technical assistance for stainings. Pavel Truschow provided technical assistance for imaging. In case a contribution is presented in form of a figure, the person who generated the data or the figure is mentioned in the figure legend.

I would like to express my gratitude towards all these people. This thesis heavily relied on their inputs. 


\section{ACKNOWLEDGEMENTS}

The past four years have been an adventurous journey. My journey through the dense network of cortical connections was as exciting and as difficult as an expedition through the unexplored rain forest. Many people accompanied me on this journey, some right from the start, others joined later. Those people carried me forward. Whenever I got lost and did not know which path to take, someone showed me the way. Whenever I saw a scary creature, someone helped me to circumvent it. Whenever a heavy rainfall soaked me, someone gave me shelter. Likewise, whenever I reached a goal, there was someone to share the joy with.

I would like to thank the leader of the expedition, my supervisor and mentor Jochen Staiger. We climbed through the deepest valleys together but I could always rely on his rope of support. He always spoke highly of me, showed confidence in my abilities and challenged me to unleash my full potential. I would like to thank Mirko Witte and Julien Guy. They were always open for discussions, helped me to design my experiments, gave me constructive feedback, provided me with the necessary animals helped me to expand and refine my skillset of techniques, ... In short, they were incredibly helpful in many aspects of my work.

I would like to thank Robin Wagener. During my Master's thesis, he taught me the basic techniques I later used in my $\mathrm{PhD}$. He awoke my passion for anatomy and was instrumental in my decision to stay in this institute for my PhD.

I would like to thank Patricia Sprysch for her help in the realms of histology. Her diligence was indispensable to the completion of this work.

I would like to thank Sandra Heinzl for help with in-situ hybridization, Pavel Truschow for his help with imaging, Alvar Prönneke for his help in graphic design, Martin Möck for writing scripts for analysis of electrophysiological data and Michael Feyerabend for the many discussions and his entertainment in and outside of the lab.

I would like to express my gratitude towards all the members of the Neuroanatomy. This institute is distinguished by a friendly environment and excellent team spirit.

I would like to thank Edward Callaway and his lab members to host me as a guest for four months. They advanced my scientific horizon and made this stay a wonderful experience.

I would like to thank Lief Fenno for being a very friendly and helpful collaborator and for sharing new viral constructs together with Yoon Seok Kim, Charu Ramakrishnan and Karl Deisseroth.

I would like to thank my room mates in San Diego, Michael Levinsky and Lukas Slivinsky, who introduced me to the California lifestyle and contributed to a great work-life balance.

I would like to thank my thesis committee members Silvio Rizzoli and Camin Dean for discussions as well as shaping a positive attitude towards my work.

I would like to thank Michael Hörner for putting the trust in me to become a good student of the neurosciences and Sandra Drube for her soft-footed coordination of my studies.

I would like to thank the GGNB for financial support and the framework to conduct this thesis. 
I would like to thank Tanvi Butola. She is an irreplaceable and inspirational role model as a scientist and as a person.

Finally, I would like to thank my family and friends. I could not have asked for better companions on my $\mathrm{PhD}$ journey.

My gratitude for how all these people helped me in my scientific endeavour is inseparable from my gratitude for how they helped me in my personal development. They made me a better scientist and a better person. Thank you.

\section{PUBLICATIONS ARISING FROM THIS THESIS}

Hafner, G., Witte, M., Guy, J., Subhashini, N., Fenno, L.E., Ramakrishnan, C., Kim, Y.S., Deisseroth, K., Oberhuber, M., Conzelmann, K.-K., et al. (2019). Mapping Brain-Wide Afferent Inputs of Parvalbumin- Expressing GABAergic Neurons in Barrel Cortex Reveals Local and Long-Range Circuit Motifs. Cell Rep. 28, 3450-3461. 


\section{SUPPLEMENTARY INFORMATION}

\subsection{Tables}

Table 1: Mean and standard deviation (SD) for input magnitude and input fraction from global areas. P-values result from paired t-tests between WT and reeler mice. Related to Figure 18.

\begin{tabular}{|c|c|c|c|c|c|c|c|}
\hline & & \multicolumn{2}{|c|}{ WT } & \multicolumn{2}{|c|}{ Reeler } & \multirow[b]{2}{*}{ p-value } & \multirow[b]{2}{*}{ adj.p-value } \\
\hline & Area & mean & SD & mean & SD & & \\
\hline \multirow{6}{*}{ 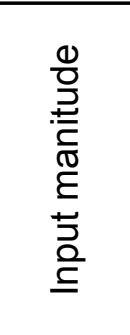 } & Ipsilateral cortical inputs & 24.96 & 8.28 & 9.72 & 4.28 & 0.0045 & $0.0268^{*}$ \\
\hline & Contralateral cortical inputs & 1.77 & 1.11 & 4.82 & 2.47 & 0.0283 & $0.0425^{*}$ \\
\hline & Cortical inputs & 26.73 & 9.33 & 14.54 & 6.19 & 0.0266 & $0.0425^{*}$ \\
\hline & Thalamic inputs & 6.35 & 2.33 & 5.30 & 1.39 & 0.3666 & 0.3666 \\
\hline & Subcortical inputs & 7.03 & 2.45 & 5.67 & 1.46 & 0.2764 & 0.3317 \\
\hline & All inputs & 33.75 & 10.29 & 20.21 & 7.03 & 0.0264 & $0.0425^{*}$ \\
\hline \multirow{6}{*}{ 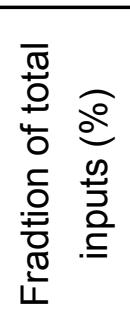 } & Ipsilateral cortical inputs & 73.49 & 8.35 & 47.53 & 8.47 & 0.0003 & $0.0020^{* *}$ \\
\hline & Contralateral cortical inputs & 4.90 & 1.46 & 22.58 & 6.29 & 0.0007 & $0.0022^{* *}$ \\
\hline & Cortical inputs & 78.39 & 8.91 & 70.11 & 8.71 & 0.1342 & 0.1611 \\
\hline & Thalamic inputs & 19.63 & 8.85 & 28.05 & 8.76 & 0.1287 & 0.1611 \\
\hline & Subcortical inputs & 21.61 & 8.91 & 29.89 & 8.71 & 0.1342 & 0.1611 \\
\hline & All inputs & 100 & 0 & 100 & 0 & & \\
\hline
\end{tabular}


Table 2: Mean and standard deviation (SD) for input fraction from individual area. P-values result from paired t-tests between WT and reeler mice. Related to Figure 20.

\begin{tabular}{|c|c|c|c|c|c|c|}
\hline \multirow[b]{2}{*}{ Area } & \multicolumn{2}{|c|}{ WT } & \multicolumn{2}{|c|}{ Reeler } & \multirow[b]{2}{*}{ p-value } & \multirow[b]{2}{*}{ adj.p-value } \\
\hline & mean & SD & mean & SD & & \\
\hline Orbital area & 0.36 & 0.14 & 0.11 & 0.07 & 0.0048 & $0.0391^{*}$ \\
\hline Primary motor $\mathrm{c}$. & 2.27 & 0.81 & 1.21 & 0.75 & 0.0414 & 0.2359 \\
\hline Secondary motor c. & 5.70 & 2.95 & 1.05 & 1.60 & 0.0099 & 0.0677 \\
\hline Motor c. & 7.97 & 3.58 & 2.26 & 2.27 & 0.0101 & 0.0101 \\
\hline Anterior cingulate $\mathrm{c}$. & 0.60 & 0.51 & 0.17 & 0.14 & 0.0940 & 0.2965 \\
\hline Retrosplenial area & 1.09 & 1.02 & 0.22 & 0.13 & 0.0897 & 0.2965 \\
\hline Primary somatosensory c. limb & 0.82 & 0.38 & 1.48 & 0.74 & 0.0933 & 0.2965 \\
\hline Primary somatosensory c. trunk & 4.11 & 3.36 & 4.78 & 1.59 & 0.6698 & 0.8322 \\
\hline Primary somatosensory c. body & 4.93 & 3.55 & 6.26 & 1.44 & 0.4253 & 0.4253 \\
\hline Secondary somatosensory c. & 27.69 & 6.48 & 20.70 & 2.49 & 0.0460 & 0.2359 \\
\hline Dorsal auditory c. & 1.82 & 1.06 & 1.83 & 1.31 & 0.9968 & 0.9968 \\
\hline Primary auditory $\mathrm{c}$. & 5.49 & 1.78 & 1.06 & 0.88 & 0.0008 & $0.0126^{*}$ \\
\hline Ventral auditory c. & 2.08 & 1.17 & 1.52 & 1.21 & 0.4385 & 0.6421 \\
\hline Auditory c. & 9.39 & 3.66 & 4.41 & 3.10 & 0.0294 & $0.0294^{*}$ \\
\hline Posterior parietal associaton c. & 12.42 & 8.53 & 7.13 & 3.19 & 0.2023 & 0.4603 \\
\hline Anteromedial visual c. & 0.97 & 0.96 & 0.13 & 0.18 & 0.0841 & 0.2965 \\
\hline Primary visual c. & 4.32 & 2.01 & 4.23 & 2.02 & 0.9422 & 0.9776 \\
\hline Anterolateral visual c. & 2.39 & 1.08 & 1.52 & 1.18 & 0.2133 & 0.4603 \\
\hline Visual c. & 7.69 & 3.00 & 5.88 & 2.75 & 0.3037 & 0.3037 \\
\hline Contralateral motor cortex & 0.15 & 0.08 & 0.16 & 0.08 & 0.8971 & 0.9776 \\
\hline Contralateral barrel cortex & 3.76 & 0.97 & 21.13 & 6.26 & 0.0009 & $0.0126^{*}$ \\
\hline Contralateral secondary somatosensory c. & 0.12 & 0.10 & 0.68 & 0.28 & 0.0031 & $0.0319^{*}$ \\
\hline $\begin{array}{l}\text { Contralateral primary somatosensory } \\
\text { body c. }\end{array}$ & 0.27 & 0.26 & 0.33 & 0.32 & 0.7318 & 0.8824 \\
\hline Anteroventral nucleus & 0.23 & 0.28 & 0.26 & 0.29 & 0.8657 & 0.9776 \\
\hline Anteromedial nucleus & 0.44 & 0.43 & 0.69 & 0.28 & 0.2715 & 0.5184 \\
\hline Ventral anterior-lateral complex & 1.04 & 0.53 & 1.96 & 1.02 & 0.0888 & 0.2965 \\
\hline Ventral posteromedial complex & 12.35 & 5.88 & 18.82 & 6.52 & 0.1016 & 0.2975 \\
\hline Ventral posterolateral complex & 0.17 & 0.13 & 0.78 & 0.25 & 0.0008 & 0.0126 \\
\hline Posterior complex & 3.37 & 1.59 & 2.82 & 1.56 & 0.5624 & 0.7439 \\
\hline Lateral dorsal nucleus & 0.11 & 0.20 & 0.33 & 0.26 & 0.1405 & 0.3599 \\
\hline Lateral posterior nucleus & 0.17 & 0.12 & 0.22 & 0.13 & 0.5612 & 0.7439 \\
\hline Rhomboid nulceus & 0.11 & 0.08 & 0.17 & 0.14 & 0.4018 & 0.6101 \\
\hline Intralaminar nucleus & 1.35 & 0.42 & 1.67 & 0.31 & 0.1669 & 0.4024 \\
\hline Lateral hypothalamic area & 0.25 & 0.11 & 0.17 & 0.13 & 0.2908 & 0.5184 \\
\hline Zona Incerta & 0.25 & 0.10 & 0.18 & 0.12 & 0.3454 & 0.5901 \\
\hline Substantia innominata & 0.14 & 0.06 & 0.13 & 0.11 & 0.9113 & 0.9776 \\
\hline Diagonal band & 0.12 & 0.06 & 0.08 & 0.04 & 0.1385 & 0.3599 \\
\hline Bed nuclei of the stria terminalis & 0.04 & 0.05 & 0.05 & 0.05 & 0.8620 & 0.9776 \\
\hline Globus pallidus & 0.68 & 0.25 & 0.69 & 0.26 & 0.9538 & 0.9776 \\
\hline Claustrum & 0.05 & 0.02 & 0.03 & 0.03 & 0.2870 & 0.5184 \\
\hline
\end{tabular}




\begin{tabular}{|l|l|l|l|l|l|l|}
\hline Amygdala & 0.04 & 0.05 & 0.02 & 0.03 & 0.3933 & 0.6101 \\
\hline Periaqueductal gray & 0.05 & 0.03 & 0.06 & 0.05 & 0.6306 & 0.8080 \\
\hline Ventral tegmental area & 0.06 & 0.03 & 0.09 & 0.07 & 0.3641 & 0.5972 \\
\hline Midbrain reticular nucleus & 0.11 & 0.14 & 0.17 & 0.11 & 0.4630 & 0.6546 \\
\hline Raphe Nuclei & 0.05 & 0.05 & 0.02 & 0.02 & 0.2293 & 0.4700 \\
\hline
\end{tabular}

\subsection{List of Abbreviations}

\begin{tabular}{|c|c|}
\hline AAV & Adeno-associated virus \\
\hline ANOVA & Analysis of variance \\
\hline Amy & Amygdala \\
\hline AUDd & Dorsal auditory area \\
\hline AUDp & Primary auditory area \\
\hline AUDV & Ventral auditory area \\
\hline AuV & Secondary auditory cortex, ventral area \\
\hline $\mathrm{BF}$ & Barrel field \\
\hline BFP & Blue fluorescent protein \\
\hline BL6 & Black six wildtype mouse strain \\
\hline CCD & Charge-coupled device \\
\hline Con/Fon & Cre and Flp recombinase dependent \\
\hline CPN & Callosal projection neuron \\
\hline DAPI & 4',6-Diamidino-2-phenylindole \\
\hline DIG & Digoxigenin \\
\hline DNA & Deoxyribonucleic acid \\
\hline EGFP & Enhanced green fluorescent protein \\
\hline EGTA & Ethylene glycol tetraacetic acid \\
\hline EnvA & Envelope A \\
\hline EPSP & Excitatory postsynaptic potential \\
\hline FAB & Fragment antigen binding \\
\hline $\mathrm{G}$ & Glycoprotein \\
\hline GABA & Gamma-aminobutyric acid \\
\hline GAD & Glutamate decarboxylase \\
\hline GFP & Green fluorescent protein \\
\hline Hepes & 4-(2-hydroxyethyl)-1-piperazineethanesulfonic acid \\
\hline HPF & Hippocampal formation \\
\hline $\lg$ & Immunglobulin \\
\hline IPSP & Inhibitory postsynaptic potential \\
\hline ISOI & Intrinsic signal optical imaging \\
\hline $\mathrm{L}$ & Layer \\
\hline LAMP5 & Lysosomal Associated Membrane Protein Family Member 5 \\
\hline MOp & Primary motor cortex \\
\hline MOs & Secondary motor cortex \\
\hline $\mathrm{ms}$ & Millisecond \\
\hline oG & Optimized glycoprotein \\
\hline
\end{tabular}




\begin{tabular}{|c|c|}
\hline $\mathrm{pA}$ & Picoampere \\
\hline PB & Phosphate buffer \\
\hline PFA & Paraformaldehyde \\
\hline $\mathrm{PO}$ & Posterior complex of the thalamus \\
\hline $\mathrm{PV}$ & Parvalbumin \\
\hline RFP & Red fluorescent protein \\
\hline RNA & Ribonucleic acid \\
\hline RSA & Retrosplenial agranular cortex \\
\hline RV & Rabies virus \\
\hline S1 & Primary somatosensory cortex \\
\hline S2 & Secondary somatosensory cortex \\
\hline SAD & Street alabama dufferin \\
\hline SD & Standard deviation \\
\hline Serpinf1 & Serpin Family F Member 1 \\
\hline Sncg & Synuclein Gamma \\
\hline SST & Somatostatin \\
\hline TB & Tris buffer \\
\hline TBS & Tris buffer saline \\
\hline TBST & Tris buffer $0.5 \%$ Triton-X 100 \\
\hline TRE & Tetracycline-responsive promoter element \\
\hline tTA & Tetracycline-controlled transactivator \\
\hline TVA & EnvA-receptor \\
\hline $\mathrm{V} 1$ & Primary visual cortex \\
\hline V2L & Secondary visual cortex, lateral area \\
\hline VISal & Anterolateral visual area \\
\hline VISam & Anteromedial visual area \\
\hline VISp & Primary visual area \\
\hline Vgat & Vesicular GABA transporter \\
\hline VGluT & Vesicular glutamate transporter \\
\hline VIP & Vasoactive intestinal polypeptide \\
\hline VPM & Ventral posteromedial nucleus \\
\hline WM & White matter \\
\hline WPRE & Woodchuck Hepatitis Virus Posttranscriptional Regulatory Element \\
\hline WT & Wild type \\
\hline 5HT3a-R & Serotonin 3a receptor \\
\hline
\end{tabular}

